\title{
Bitableaux Bases for some Garsia-Haiman Modules and Other Related Modules
}

\author{
E. E. Allen * \\ Department of Mathematics \\ Wake Forest University \\ Winston-Salem, NC 27109 \\ allene@wfu.edu
}

Submitted: October 6, 2000 ; Accepted: April 5, 2002.

MR Subject Classifications: 05E05, 05E10

\begin{abstract}
For certain subsets $S$ and $T$ of $\mathcal{A}=\{\cdots,(0,2),(0,1),(0,0),(1,0),(2,0), \cdots\}$ and factor spaces $\mathbb{C}_{S}[X, Y], \mathbb{C}_{S, T}^{+}[X, Y, Z, W]$ and $\mathbb{C}_{S, T}^{-}[X, Y, Z, W]$, bitableaux bases are constructed that are indexed by pairs of standard tableaux and sequences in the collections $\Upsilon_{\psi_{S}}$ and $\Upsilon_{\psi_{T}}$. These bases give combinatorial interpretations to the appropriate Hilbert series of these spaces as well as the graded character of $\mathbb{C}_{S}[X, Y]$.

The factor space $\mathbb{C}_{S}[X, Y]$ is an analogue of the coinvariant ring of a polynomial ring in two sets of variables. $\mathbb{C}_{S, T}^{+}[X, Y, Z, W]$ and $\mathbb{C}_{S, T}^{-}[X, Y, Z, W]$ are analogues of coinvariant spaces in symmetric and skew-symmetric polynomial settings, respectively. The elements of the bitableaux bases are appropriately defined images in the polynomial spaces of bipermanents. The combinatorial interpretations of the respective Hilbert series and graded characters are given by statistics based on cocharge tableaux.

Additionally, it is shown that the Hilbert series and graded characters factor nicely. One of these factors gives the Hilbert series of a collection of Schur functions $s_{\lambda / \mu}$ where $\mu$ varies in an appropriately defined $\lambda$.
\end{abstract}

* Thanks to all of the wonderful editors of this journal! 


\section{Introduction.}

Let $\mathcal{A}$ be the alphabet

$$
\mathcal{A}=\{(f, g): f \text { and } g \text { are nonnegative integers such that } f g=0\} .
$$

Specifically,

$$
\mathcal{A}=\{\cdots,(0,3),(0,2),(0,1),(0,0),(1,0),(2,0),(3,0), \cdots\}
$$

The elements of $\mathcal{A}$ are the coordinates $(i, k)$, of the cells of the hookshape in the first quadrant of the plane as shown:

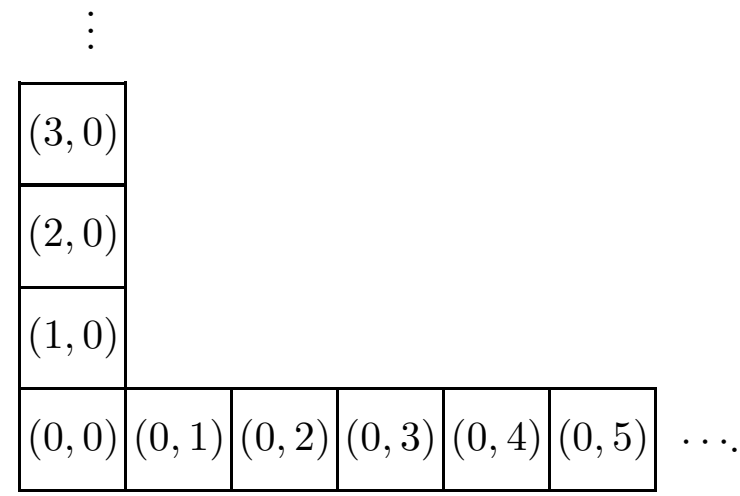

We will say that

$$
\left(a_{1}, b_{1}\right)<_{\mathcal{A}}\left(a_{2}, b_{2}\right)
$$

if and only if $a_{1}-b_{1}<a_{2}-b_{2}$.

Let $\mathbb{C}[X, Y, Z, W]$ denote the polynomial ring with complex coefficients in $X=$ $\left\{x_{1}, x_{2}, \cdots, x_{n}\right\}, Y=\left\{y_{1}, y_{2}, \cdots, y_{n}\right\}, Z=\left\{z_{1}, z_{2}, \cdots, z_{n}\right\}$ and $W=\left\{w_{1}, w_{2}, \cdots, w_{n}\right\}$. Given a subset

$$
S=\left\{\left(a_{1}, b_{1}\right),\left(a_{2}, b_{2}\right), \cdots,\left(a_{n}, b_{n}\right)\right\} \subset \mathcal{A}
$$

of the alphabet $\mathcal{A}$ listed in increasing order with respect to $<_{\mathcal{A}}$, we define $M_{S}$ to be the $n \times n$ matrix

$$
M_{S}=\left(x_{i}^{a_{k}} y_{i}^{b_{k}}\right)_{1 \leq i, k \leq n}
$$

and $\Delta_{S}(X, Y)$ to be the determinant of $M_{S}$. Let $\partial_{x_{i}}$ denote the partial differential operator with respect to $x_{i}$. With $P(X, Y) \in \mathbb{C}[X, Y]$, we will set

$$
P\left(\partial_{X}, \partial_{Y}\right)=P\left(\partial_{x_{1}}, \partial_{x_{2}}, \cdots, \partial_{x_{n}}, \partial_{y_{1}}, \partial_{y_{2}}, \cdots, \partial_{y_{n}}\right)
$$

Setting $\mathcal{I}_{S}(X, Y)$ to be the ideal

$$
\mathcal{I}_{S}(X, Y)=\left\{P(X, Y) \in \mathbb{C}[X, Y]: P\left(\partial_{X}, \partial_{Y}\right) \Delta_{S}(X, Y)=0\right\}
$$


we define $\mathbb{C}_{S}[X, Y]$ to be the polynomial quotient ring

$$
\mathbb{C}_{S}[X, Y]=\mathbb{C}[X, Y] / \mathcal{I}_{S}(X, Y)
$$

The rings $\mathbb{C}_{S}[X, Y]$ are called Garsia-Haiman modules. A. Garsia and M. Haiman introduced modules of this type to study the $q, t$-Kostka coefficients (see [10]).

The action of $\sigma \in S_{n}$ (where $S_{n}$ denotes the symmetric group on $n$ letters) on the polynomial

$$
P(X, Y, Z, W) \in \mathbb{C}[X, Y, Z, W]
$$

is defined by setting

$$
\begin{array}{rl}
\sigma_{X, Y, Z, W} & P\left(x_{1}, x_{2}, \cdots, x_{n}, y_{1}, y_{2}, \cdots, y_{n}, z_{1}, \cdots, z_{n}, w_{1}, \cdots, w_{n}\right) \\
= & P\left(x_{\sigma_{1}}, x_{\sigma_{2}}, \cdots, x_{\sigma_{n}}, y_{\sigma_{1}}, y_{\sigma_{2}}, \cdots, y_{\sigma_{n}}, z_{\sigma_{1}}, \cdots, z_{\sigma_{n}}, w_{\sigma_{1}}, \cdots, w_{\sigma_{n}}\right), \\
\sigma_{X, Y} & P\left(x_{1}, x_{2}, \cdots, x_{n}, y_{1}, y_{2}, \cdots, y_{n}, z_{1}, \cdots, z_{n}, w_{1}, \cdots, w_{n}\right) \\
= & P\left(x_{\sigma_{1}}, x_{\sigma_{2}}, \cdots, x_{\sigma_{n}}, y_{\sigma_{1}}, y_{\sigma_{2}}, \cdots, y_{\sigma_{n}}, z_{1}, \cdots, z_{n}, w_{1}, \cdots, w_{n}\right)
\end{array}
$$

and

$$
\begin{array}{rl}
\sigma_{Z, W} & P\left(x_{1}, x_{2}, \cdots, x_{n}, y_{1}, y_{2}, \cdots, y_{n}, z_{1}, \cdots, z_{n}, w_{1}, \cdots, w_{n}\right) \\
\quad= & P\left(x_{1}, x_{2}, \cdots, x_{n}, y_{1}, y_{2}, \cdots, y_{n}, z_{\sigma_{1}}, \cdots, z_{\sigma_{n}}, w_{\sigma_{1}}, \cdots, w_{\sigma_{n}}\right) .
\end{array}
$$

Note that the subscripts $X, Y, Z, W, X, Y$ and $Z, W$ denote the sets of variables on which $\sigma$ acts.

For subsets $S$ and $T$ of $\mathcal{A}$, let

$$
\begin{aligned}
\mathbb{C}^{S_{n}} & {[X, Y, Z, W]=\left\{P \in \mathbb{C}[X, Y, Z, W]: \sigma_{X, Y, Z, W} P=P \forall \sigma \in S_{n}\right\}, } \\
& \mathcal{I}_{S, T}^{+}(X, Y, Z, W) \\
= & \left\{P \in \mathbb{C}^{S_{n}}[X, Y, Z, W]: P\left(\partial_{X}, \partial_{Y}, \partial_{Z}, \partial_{W}\right) \Delta_{S}(X, Y) \Delta_{T}(Z, W)=0\right\}
\end{aligned}
$$

and

$$
\mathbb{C}_{S, T}^{+}[X, Y, Z, W]=\mathbb{C}^{S_{n}}[X, Y, Z, W] / \mathcal{I}_{S, T}^{+}(X, Y, Z, W) .
$$

Analogously, with $\operatorname{sgn}(\sigma)$ denoting the sign of the permutation $\sigma$, let

$$
\begin{aligned}
\mathbb{C}^{-}[X, Y, Z, W]=\left\{P \in \mathbb{C}[X, Y, Z, W]: \sigma_{X, Y, Z, W} P=\operatorname{sgn}(\sigma) P \forall \sigma \in S_{n}\right\}, \\
\mathcal{I}_{S, T}^{-}(X, Y, Z, W) \\
=\left\{P \in \mathbb{C}^{-}[X, Y, Z, W]: P\left(\partial_{X}, \partial_{Y,} \partial_{Z}, \partial_{W}\right) \Delta_{S}(X, Y) \Delta_{T}(Z, W)=0\right\}
\end{aligned}
$$


and

$$
\mathbb{C}_{S, T}^{-}[X, Y, Z, W]=\mathbb{C}^{-}[X, Y, Z, W] / \mathcal{I}_{S, T}^{-}(X, Y, Z, W)
$$

It should be noted that $\mathbb{C}^{S_{n}}[X, Y, Z, W]$ and $\mathbb{C}_{S, T}^{+}[X, Y, Z, W]$ are rings. $\mathbb{C}_{S, T}^{-}[X, Y, Z, W]$ is not closed under multiplication and hence is not a ring. Thus we will be considering $\mathbb{C}_{S, T}^{-}[X, Y, Z, W]$ simply as a module.

In this paper, we construct bases for $\mathbb{C}_{S}[X, Y], \mathbb{C}_{S, T}^{+}[X, Y, Z, W]$ and $\mathbb{C}_{S, T}^{-}[X, Y, Z, W]$ (for certain general classes of $S$ and $T$ that we shall call dense) that are indexed by pairs of standard tableaux and sequences in the collections $\Upsilon_{\psi_{S}}$ and $\Upsilon_{\psi_{T}}$. In Section Two, we introduce tableaux, bitableaux, bipermanents and bideterminants. In Section Three, we define dense Garsia-Haiman Modules. The bases for $\mathbb{C}_{S}[X, Y], \mathbb{C}_{S, T}^{+}[X, Y, Z, W]$ and $\mathbb{C}_{S, T}^{-}[X, Y, Z, W]$ are constructed in Sections Three, Four and Five, respectively, using bipermanents and bideterminants. Specifically, with $\mathcal{S} \mathcal{T}_{n}$ denoting the collection of standard tableaux with $n$ cells, $\operatorname{sh}(Q)$ denoting the shape of the tableaux $Q, \mathcal{C O}_{S}$ denoting a collection of cocharge tableaux and $[Q, C]_{p e r},[U, V]_{p e r}^{+}$and $[U, V]_{p e r}^{-}$denoting certain images of bipermanents in the factor spaces $\mathbb{C}_{S}[X, Y], \mathbb{C}_{S, T}^{+}[X, Y, Z, W]$ and $\mathbb{C}_{S, T}^{-}[X, Y, Z, W]$, respectively, we will prove the following theorems:

\section{Theorem 1.1.}

If $S$ is dense then the collection

$$
\mathcal{B}_{\psi_{S}}=\left\{[Q, C]_{\text {per }}: Q \in \mathcal{S T}_{n}, C \in \mathcal{C} \mathcal{O}_{S} \text { and } \operatorname{sh}(Q)=\operatorname{sh}(C)\right\}
$$

is a basis for $\mathbb{C}_{S}[X, Y]$ with coefficients from $\mathbb{C}$.

\section{Theorem 1.2.}

If $S$ and $T$ are dense then

$$
\mathcal{B S} \mathcal{Q}_{S, T}=\left\{[U, V]_{\text {per }}^{+}: U \in \mathcal{C} \mathcal{O}_{S}, V \in \mathcal{C O}_{T} \text { and } \operatorname{sh}(U)=\operatorname{sh}(V)\right\}
$$

is a basis for $\mathbb{C}_{S, T}^{+}[X, Y, Z, W]$ with coefficients from $\mathbb{C}$.

\section{Theorem 1.3.}

If $S$ and $T$ are dense then the collection

$$
\mathcal{B A} \mathcal{Q}_{S, T}=\left\{[U, V]_{p e r}^{-}: U \in \mathcal{C O}_{S}, V \in \mathcal{C O}_{T} \text { and } \operatorname{sh}(U)=\operatorname{sh}(V)\right\}
$$

is a basis for $\mathbb{C}_{S, T}^{-}[X, Y, Z, W]$ with coefficients from $\mathbb{C}$.

Note that these theorems are Theorem 3.8, Theorem 4.4 and Theorem 5.7, respectively. 
If $R_{u_{1}, u_{2}, u_{3}, u_{4}}$ is a homogeneous subspace of dimension $u_{1}$ in $X, u_{2}$ in $Y, u_{3}$ in $Z$ and $u_{4}$ in $W$, then we define the Hilbert series $\mathcal{H}(R)$ to be

$$
\mathcal{H}(R)=\sum_{u_{1}, u_{2}, u_{3}, u_{4}} \operatorname{dim}\left(R_{u_{1}, u_{2}, u_{3}, u_{4}}\right) t^{u_{1}} q^{u_{2}} r^{u_{3}} s^{u_{4}} .
$$

These bases imply combinatorial interpretations for the Hilbert series of $\mathbb{C}_{S}[X, Y]$, $\mathbb{C}_{S, T}^{+}[X, Y, Z, W]$ and $\mathbb{C}_{S, T}^{-}[X, Y, Z, W]$.

\section{Theorem 1.4.}

If $S$ is dense then the Hilbert series $\mathcal{H}\left(\mathbb{C}_{S}[X, Y]\right)$ is given by

$$
\mathcal{H}\left(\mathbb{C}_{S}[X, Y]\right)=\sum_{\lambda \vdash n} h_{\lambda} \sum_{M \in \mathcal{S} \mathcal{T}_{\lambda}} \sum_{\rho \in \Upsilon_{\psi_{S}}} t^{\left|C_{\rho, 1}(M)\right|} q^{\left|C_{\rho, 2}(M)\right|}
$$

where $\mathcal{S} \mathcal{T}_{\lambda}$ denotes the collection of standard tableaux of shape $\lambda, h_{\lambda}$ denotes the number of standard tableaux of shape $\lambda, \Upsilon_{\psi_{S}}$ is a collection of sequences defined in equation (3.4) and $\left|C_{\rho, 1}(M)\right|$ and $\left|C_{\rho, 2}(M)\right|$ denote the sums of the first and second coordinates, respectively, of the entries of $C_{\rho}(M)$.

\section{Theorem 1.5.}

If $S$ and $T$ are dense then the Hilbert series $\mathcal{H}\left(\mathbb{C}_{S, T}^{+}[X, Y, Z, W]\right)$ for $\mathbb{C}_{S, T}^{+}[X, Y, Z, W]$ is given by

$$
\begin{aligned}
& \mathcal{H}\left(\mathbb{C}_{S, T}^{+}[X, Y, Z, W]\right) \\
& =\sum_{\lambda \vdash n} \sum_{\rho \in \Upsilon_{\psi_{S}}} \sum_{\rho^{\prime} \in \Upsilon_{\psi_{T}}} \sum_{(M, N) \in \mathcal{S} \mathcal{T}_{\lambda} \times \mathcal{S} \mathcal{T}_{\lambda}} t^{\left|C_{\rho, 1}(M)\right|} q^{\left|C_{\rho, 2}(M)\right|} r^{\left|C_{\rho^{\prime}, 1}(N)\right|} s^{\left|C_{\rho^{\prime}, 2}(N)\right|}
\end{aligned}
$$

where $\mathcal{S} \mathcal{T}_{\lambda}$ denotes the collection of standard tableaux of shape $\lambda$ and $\Upsilon_{\psi_{S}}$ and $\Upsilon_{\psi_{T}}$ are collections of sequences defined in equation (3.4).

\section{Theorem 1.6.}

If $S$ and $T$ are dense the Hilbert series $\mathcal{H}\left(\mathbb{C}_{S, T}^{-}[X, Y, Z, W]\right)$ for $\mathbb{C}_{S, T}^{-}[X, Y, Z, W]$ is given by

$$
\begin{aligned}
& \mathcal{H}\left(\mathbb{C}_{S, T}^{-}[X, Y, Z, W]\right) \\
& =\sum_{\lambda \vdash n} \sum_{\rho \in \Upsilon_{\psi_{S}}} \sum_{\rho^{\prime} \in \Upsilon_{\psi_{T}}} \sum_{(M, N) \in \mathcal{S} \mathcal{T}_{\lambda} \times \mathcal{S} \mathcal{T}_{\lambda}} t^{\left|C_{\rho, 1}(M)\right|} q^{\left|C_{\rho, 2}(M)\right|} r^{\left|C_{\rho^{\prime}, 1}\left(N^{t}\right)\right|} s^{\left|C_{\rho^{\prime}, 2}\left(N^{t}\right)\right|}
\end{aligned}
$$

where $\mathcal{S} \mathcal{T}_{\lambda}$ denotes the collection of standard tableaux of shape $\lambda, \Upsilon_{\psi_{S}}$ and $\Upsilon_{\psi_{T}}$ are collections of sequences defined in equation (3.4) and $N^{t}$ denotes the transpose of the standard tableau $N$. 
Note that the above three theorems are Corollary 3.10, Corollary 4.5 and Corollary 5.8 , respectively. Furthermore, since the action of $S_{n}$ on a basis for $\mathbb{C}_{S}[X, Y]$ can be described in terms of irreducible representations of $S_{n}$, we will be able to compute the graded character of the spaces $\mathbb{C}_{S}[X, Y]$ (see Corollary 3.12).

\section{Theorem 1.7.}

With $S$ dense, the graded character char ${ }_{q, t}\left(\mathbb{C}_{S}[X, Y]\right)$ of $\mathbb{C}_{S}[X, Y]$ is given by:

$$
\operatorname{char}_{q, t}\left(\mathbb{C}_{S}[X, Y]\right)=\sum_{\lambda \vdash n} \chi^{\lambda} \sum_{\rho \in \Upsilon_{\psi_{S}}} \sum_{M \in \mathcal{S} \mathcal{T}_{\lambda}} t^{\left|C_{\rho, 1}(M)\right|} q^{\left|C_{\rho, 2}(M)\right|}
$$

where $\mathcal{S T}_{\lambda}$ denotes the collection of standard tableaux of shape $\lambda, \Upsilon_{\psi_{S}}$ is a collection of sequences defined in equation (3.4), and $\left|C_{\rho, 1}(M)\right|$ and $\left|C_{\rho, 2}(M)\right|$ denote the sum of the first and second coordinates, respectively, of the entries of $C_{\rho}(N)$ and $\chi^{\lambda}$ denotes the irreducible $S_{n}$ character corresponding to shape $\lambda$.

Note that in Theorem 1.4, Theorem 1.5, Theorem 1.6 and Theorem 1.7, due to the construction of the cocharge statistic, we are able to factor the resulting Hilbert series as well as the graded character (see the theorems in the respective chapters). Additionally, one of the factors of these polynomials turns out to be the Hilbert series of a collection of skew Schur functions $s_{\lambda / \mu}$ as $\mu$ varies in a partition $\lambda$ that corresponds to to a dense set $S$ (see Corollary 3.13).

It should be noted that $\mathbb{C}_{S}[X, Y], \mathbb{C}_{S, T}^{+}[X, Y, Z, W]$ and $\mathbb{C}_{S, T}^{-}[X, Y, Z, W]$ are generalizations of some well-studied modules. For example, if

$$
S=\{(0,0),(1,0), \cdots,(n-1,0)\}
$$

then $\Delta_{S}(X, Y)$ is the Vandermonde determinant in the variables $\left\{x_{1}, x_{2}, \cdots, x_{n}\right\}$ and $\mathcal{I}_{S}(X, Y)$ is the ideal generated by the elementary symmetric functions

$$
e_{k}=\sum_{1 \leq i_{1}<i_{2}<\cdots<i_{k} \leq n} x_{i_{1}} x_{i_{2}} \cdots x_{i_{k}}
$$

for $1 \leq k \leq n$ and the monomials $\left\{y_{1}, y_{2}, \cdots, y_{n}\right\}$. In this case, $\mathbb{C}_{S}[X, Y]$ is the ring of coinvariants in the variables $X=\left\{x_{1}, x_{2}, \cdots, x_{n}\right\}$ associated with the symmetric group $S_{n}$. Bases for $\mathbb{C}_{S}[X, Y]$ are given in [13] (in which it is shown that the collection $\left\{x_{1}^{\epsilon_{1}} x_{2}^{\epsilon_{2}} \cdots x_{n}^{\epsilon_{n}}: 0 \leq \epsilon_{i} \leq i-1\right\}$ is a basis), in [8] (in which a basis is constructed using the descent monomials) and [14] and [15] (in which it is shown that the Schubert Polynomials form a basis). $\mathbf{C}_{S, S}^{+}$has been shown to have a basis closely related to the descent monomials (see [2] or [16]). A. Garsia computed the Hilbert series of $\mathbf{C}_{S, S}^{-}$in [9]. It should be noted that all of the above results are with the collection $S$ as given in (1.12). The results of this paper are related to a much larger class of collections. Additionally, the construction of a basis for $\mathbf{C}_{S, T}^{-}$(for general classes of dense sets $S$ 
and $T$ ) corresponds to constructing a basis in a noncommutative letter place algebra. Specifically, these factor spaces $\mathbf{C}_{S, T}^{-}$are analogues of coinvariant rings in an exterior algebra setting. The proofs of these theorems include algorithms for expanding elements of these modules in terms of the respective bases.

M. Haiman recently announced a proof showing that the dimension of $\mathbb{C}_{S}[X, Y]$ for classes of $S$ related to partitions have dimension $n$ ! (see [12]). This particular paper deals with a different type of class for $S$ (specifically dense sets), construction of appropriate bases for $\mathbb{C}_{S}[X, Y]$ and its relation to the rings $\mathbb{C}_{S, T}^{+}[X, Y, Z, W]$ and $\mathbb{C}_{S, T}^{-}[X, Y, Z, W]$.

\section{Bitableaux, Bipermanents and Bideterminants}

General references for much of the material in this section (specifically, letter place algebras, bitableaux, bideterminants and bipermanents) can be found in [7] or [11]. Let $\lambda=\left(\lambda_{1}, \lambda_{2}, \cdots, \lambda_{k}\right)$ be a partition of $n$. In other words, $\lambda_{1} \geq \lambda_{2} \geq \cdots \geq \lambda_{k}>0$ and $n=\lambda_{1}+\lambda_{2}+\cdots+\lambda_{k}$. This is commonly denoted by $\lambda \vdash n$. We will use the French notation for depicting Ferrers diagrams and tableaux. A Ferrers diagram of shape $\lambda$ has $\lambda_{1}$ cells in the first row, and continuing north, has $\lambda_{2}$ cells in the second row, etc. For example,

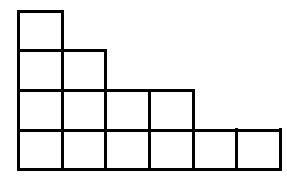

is a Ferrers diagram of shape $(6,4,2,1)$. A tableau of shape $\lambda$ is a Ferrers diagram of shape $\lambda$ where each cell contains an entry from some alphabet. A tableau $Q$ of shape $\lambda$ is said to be injective if the alphabet is $\{1,2, \cdots, n\}$ and each of the letters appear exactly once as entries in the cells of $Q$. (Note that if $Q$ has shape $\lambda \vdash n$ then $Q$ has exactly $n$ cells.) We will say that a tableau $Q$ is standard if $Q$ is injective, $\operatorname{sh}(Q)=\lambda$ where $\lambda \vdash n$ and the entries strictly increase from west to east (left to right) and from south to north (bottom to top). We will say that a tableau $Q$ is column-strict if the entries of $Q$ increase weakly from west to east but increase strictly from south to north. A tableau $Q$ is said to be row-strict if the entries increase strictly in the rows from west to east and increase weakly in the columns from south to north. We will denote the collections of all column-strict tableaux and row-strict tableaux with entries from the alphabet $\mathcal{A}$ and exactly $n$ cells by $\mathcal{C S}_{n}$ and $\mathcal{R S}_{n}$, respectively. The set of standard tableaux with entries $\{1,2, \cdots, n\}$ will be denoted by $\mathcal{S} \mathcal{T}_{n}$. We will denote the shape of a tableau $Q$ (i.e., the shape of the underlying Ferrers diagram) as $s h(Q)$.

The column sequence $\operatorname{cs}(Q)$ of a tableau $Q$ is a listing of the entries of $Q$ from south to north (bottom to top) in each column starting with the column farthest west (left) and continuing east (right). Analogously, the row sequence $r s(Q)$ of a tableau $Q$ is a listing of the entries of $Q$ from west to east in each column starting with the row farthest south and continuing north. 
If $Q$ is a tableau of shape $\lambda=\left(\lambda_{1}, \lambda_{2}, \cdots, \lambda_{k}\right)$ and $R$ is a tableau of shape $\mu=$ $\left(\mu_{1}, \mu_{2}, \cdots, \mu_{j}\right)$, we will say that $Q$ is longer than $R$ if and only if $\lambda$ is lexicographically larger than $\mu$. Similarly, we will say that $Q$ is higher than $R$ if and only if the conjugate partition $\lambda^{\prime}$ is lexicographically larger than the conjugate partition $\mu^{\prime}$. The transpose $Q^{t}$ of a tableau $Q$ of shape $\lambda$ is the tableau of shape $\lambda^{\prime}$ obtained by reflecting $Q$ along its diagonal.

\section{Example}

Let

$$
Q=\begin{array}{lllll}
6 & 8 & & & \\
3 & 5 & 7 & \\
1 & 2 & 4 & 9
\end{array} .
$$

Then

$$
\begin{array}{r}
9 \\
Q^{t}=\begin{array}{rll}
4 & 7 & \\
2 & 5 & 8 \\
1 & 3 & 6 \\
r s(Q)=1,2,4,9,3,5,7,6,8
\end{array}
\end{array}
$$

and

$$
\operatorname{cs}(Q)=1,3,6,2,5,8,4,7,9
$$

Let $\mathcal{L P}$ be the algebra of polynomials over $\mathbb{C}$ in the indeterminants $\left(a_{i} \mid b_{k}\right)$ where $a_{i}$ and $b_{k}$ are elements from some alphabets $\mathcal{A L}$ and $\mathcal{B L}$ respectively. $\mathcal{L} \mathcal{P}$ is called the letter place algebra. Note that the letter place algebra $\mathcal{L P}$ is commutative. Specifically, we have that

$$
\begin{aligned}
& \left(a_{1} \mid b_{1}\right)\left(a_{2} \mid b_{2}\right)\left(a_{3} \mid b_{3}\right) \cdots\left(a_{n} \mid b_{n}\right) \\
= & \left(a_{\sigma(1)} \mid b_{\sigma(1)}\right)\left(a_{\sigma(2)} \mid b_{\sigma(2)}\right)\left(a_{\sigma(3)} \mid b_{\sigma(3)}\right) \cdots\left(a_{\sigma(n)} \mid b_{\sigma(n)}\right)
\end{aligned}
$$

for all $\sigma \in S_{n}$ (the symmetric group). Note that this implies

$$
\left(a_{\sigma(1)} \mid b_{1}\right)\left(a_{\sigma(2)} \mid b_{2}\right) \cdots\left(a_{\sigma(n)} \mid b_{n}\right)=\left(a_{1} \mid b_{\sigma^{-1}(1)}\right)\left(a_{2} \mid b_{\sigma^{-1}(2)}\right) \cdots\left(a_{n} \mid b_{\sigma^{-1}(n)}\right) .
$$

Let $I$ be an injective tableau of shape $\lambda=\left(\lambda_{1}, \cdots, \lambda_{k}\right)$. Let $R_{i}(1 \leq i \leq k)$ denote the collection of integers in the $i^{\text {th }}$ row of $I$. Similarly, let $D_{i}(1 \leq i \leq j)$ denote the collection of integers in the $i^{\text {th }}$ column of $I$. Set

$$
R(I)=S_{R_{1}} \times S_{R_{2}} \times \cdots \times S_{R_{k}}
$$

and

$$
D(I)=S_{D_{1}} \times S_{D_{2}} \times \cdots \times S_{D_{j}}
$$


where $S_{R_{i}}$ and $S_{D_{i}}$ denote the symmetric group on the collections of elements $R_{i}$ and $D_{i}$ respectively. Define, in the group algebra $\mathbb{C}\left[S_{n}\right]$,

$$
P(I)=\sum_{\sigma \in R(I)} \sigma
$$

and

$$
N(I)=\sum_{\sigma \in D(I)} \operatorname{sgn}(\sigma) \sigma
$$

Similarly, let $\left[i_{1}, i_{2}, \cdots, i_{k}\right]$ and $\left[i_{1}, i_{2}, \cdots, i_{k}\right]^{\prime}$ denote the formal sums in the group algebra $\mathbb{C}\left[S_{n}\right]$ of $S_{n}$,

$$
\left[i_{1}, i_{2}, \cdots, i_{k}\right]=\sum_{\sigma \in S_{\left\{i_{1}, i_{2}, \cdots, i_{k}\right\}}} \sigma
$$

and

$$
\left[i_{1}, i_{2}, \cdots, i_{k}\right]^{\prime}=\sum_{\sigma \in S_{\left\{i_{1}, i_{2}, \cdots, i_{k}\right\}}} \operatorname{sgn}(\sigma) \sigma .
$$

Now, given two tableaux, $U$ and $V$, of the same shape $\lambda$ and an injective tableau $I$ (also of shape $\lambda$ ), let $u_{i}$ and $v_{i}$ be the entries in $U$ and $V$ that correspond to the cell containing $i$ in $I$, respectively. The bideterminant $(U, V)_{\text {det }}$ is defined to be

$$
\begin{aligned}
(U, V)_{\text {det }} & =N(I)\left(u_{1} \mid v_{1}\right) \cdots\left(u_{n} \mid v_{n}\right) \\
& =\sum_{\sigma \in D(I)} \operatorname{sgn}(\sigma) \sigma\left(u_{1} \mid v_{1}\right) \cdots\left(u_{n} \mid v_{n}\right) \\
& =\sum_{\sigma \in D(I)} \operatorname{sgn}(\sigma)\left(u_{\sigma(1)} \mid v_{1}\right) \cdots\left(u_{\sigma(n)} \mid v_{n}\right)
\end{aligned}
$$

and the bipermanent $(U, V)_{\text {per }}$ is defined as

$$
\begin{aligned}
(U, V)_{p e r} & =P(I)\left(u_{1} \mid v_{1}\right) \cdots\left(u_{n} \mid v_{n}\right) \\
& =\sum_{\sigma \in R(I)}\left(u_{\sigma(1)} \mid v_{1}\right) \cdots\left(u_{\sigma(n)} \mid v_{n}\right) .
\end{aligned}
$$

The content $\operatorname{con}(U, V)$ of a bideterminant $(U, V)_{\text {det }}$ (or a bipermanent $(U, V)_{\text {per }}$ ) is

$$
\operatorname{con}(U, V)=\left(\left(\alpha_{1}, \alpha_{2}, \cdots, \alpha_{k}\right),\left(\beta_{1}, \beta_{2}, \cdots, \beta_{j}\right)\right)
$$

where $\alpha_{i}$ denotes the number of entries of $a_{i}$ in $U$ and $\beta_{h}$ denotes the number of entries of $b_{h}$ in $V$. We will say that the bitableaux

$$
(U, V)<_{s^{\prime} c}(M, Q),
$$


where $U$ and $V$ have shape $\lambda$ and $M$ and $Q$ have shape $\mu$ when

1. $\lambda^{\prime}<_{L} \mu^{\prime}$ (where $>_{L}$ denotes the lexicographic ordering); or

2. if $\lambda=\mu$ then $\operatorname{cs}(U) \operatorname{cs}(V)>_{L} \operatorname{cs}(M) \operatorname{cs}(Q)$.

The following theorem may be found in [7] or [11]. The proof of Theorem 2.1 included here (which was pointed out to this author by A. Garsia) is different than the proof found in either [7] or [11]. This particular proof is included since it provides an algorithm that will be useful later in this development.

\section{Theorem 2.1.}

The collections

$$
\mathcal{C S D}=\left\{(M, Q)_{\text {det }}: M, Q \in \mathcal{C S}, \operatorname{sh}(M)=\operatorname{sh}(Q)\right\}
$$

and

$$
\mathcal{C S P}=\left\{(M, Q)_{p e r}: M, Q \in \mathcal{C S}, \operatorname{sh}(M)=\operatorname{sh}(Q)\right\},
$$

where $\mathcal{C S}$ denotes the collection of column-strict tableaux, linearly span the letter place algebra $\mathcal{L P}$ with coefficients from $\mathbb{C}$.

\section{Proof}

Note that

$$
\left(u_{1} \mid v_{1}\right)\left(u_{2} \mid v_{2}\right)\left(u_{3} \mid v_{3}\right) \cdots\left(u_{n} \mid v_{n}\right)=(U, V)_{d e t}
$$

where

$$
\begin{gathered}
U=u_{1} u_{2} u_{3} \cdots u_{n} \\
V=v_{1} v_{2} v_{3} \cdots v_{n}
\end{gathered}
$$

and

$$
I=\begin{array}{lllll}
1 & 2 & 3 & \cdots & n
\end{array}
$$

Thus $\mathcal{L} \mathcal{P}$ is spanned by the collection

$$
\left\{(U, V)_{\text {det }}: \operatorname{sh}(U)=\operatorname{sh}(V)\right\}
$$

where $U$ and $V$ are not necessarily column-strict.

Suppose that $U$ and $V$ are two tableaux of shape $\lambda$, suppose $I$ is an injective tableau of shape $\lambda$ and let $u_{i}$ and $v_{i}$ be the entries in $U$ and $V$ that correspond to the cell containing $i$ in $I$ respectively. Recall that if

$$
\left\{i_{j, 1}, i_{j, 2}, \cdots, i_{j, k_{j}}\right\}
$$

is the $j^{\text {th }}$ column of $I$, (where $I$ has $h$ columns and thus $1 \leq j \leq h$ ) then

$$
N(I)=\left[i_{1,1}, i_{1,2}, \cdots, i_{1, k_{1}}\right]^{\prime}\left[i_{2,1}, i_{2,2}, \cdots, i_{2, k_{2}}\right]^{\prime} \cdots\left[i_{h, 1}, i_{h, 2}, \cdots, i_{h, k_{h}}\right]^{\prime}
$$


and

$$
(U, V)_{\text {det }}=N(I)\left(u_{1} \mid v_{1}\right)\left(u_{2} \mid v_{2}\right)\left(u_{3} \mid v_{3}\right) \cdots\left(u_{n} \mid v_{n}\right)
$$

For a given content $\left(\left(\alpha_{1}, \alpha_{2}, \cdots, \alpha_{k}\right),\left(\beta_{1}, \beta_{2}, \cdots, \beta_{j}\right)\right)$, let $(U, V)$ be a bitableau such that

$$
\operatorname{con}(U, V)=\left(\left(\alpha_{1}, \alpha_{2}, \cdots, \alpha_{k}\right),\left(\beta_{1}, \beta_{2}, \cdots, \beta_{j}\right)\right)
$$

and $\operatorname{sh}(U)=\operatorname{sh}(V)=1^{n}$. Specifically, without loss of generality we may assume that

$$
U=\begin{gathered}
u_{n} \\
u_{n-1} \\
\vdots \\
u_{2} \\
u_{1}
\end{gathered}
$$

and

$$
V=\begin{gathered}
v_{n} \\
v_{n-1} \\
\vdots \\
v_{2} \\
v_{1}
\end{gathered}
$$

where $u_{i} \leq u_{i+1}$ and $v_{i} \leq v_{i+1}$ for $1 \leq i \leq n-1$. If $u_{i}=u_{i+1}$ or $v_{i}=v_{i+1}$ for any $i$, then $(U, V)_{d e t}=0$ and $(U, V)_{d e t}$ is in the linear span of $\mathcal{C S D}$. If $u_{i}<u_{i+1}$ and $v_{i}<v_{i+1}$ for all $i$, then $(U, V)_{\text {det }} \in \mathcal{C S D}$.

Let $(U, V)$ be the largest bitableau with respect to $>_{s^{\prime} c}$ such that $(U, V)_{\text {det }}$ cannot be written as a linear combination of

$$
\mathcal{C S D}_{n}=\left\{(M, Q)_{\text {det }}: M \in \mathcal{C S}_{n}, Q \in \mathcal{C S}_{n}, \operatorname{sh}(U)=\operatorname{sh}(V)\right\} .
$$

Without loss of generality, we may assume that even though at least one of $U$ and $V$ is not column-strict, the entries of the columns of both $U$ and $V$ increase strictly from south to north (or bottom to top). Suppose $U$ is not column-strict. Thus, let us suppose that $(j, m)$ is the smallest lexicographic pair of integers such that $u_{j, m}>u_{j+1, m}$. Note that if $u_{j, m} \leq u_{j+1, m}$ for all $j$ and $m$ then $U$ is column-strict. Essentially, in columns $j$ and $j+1$ of $U$ we have

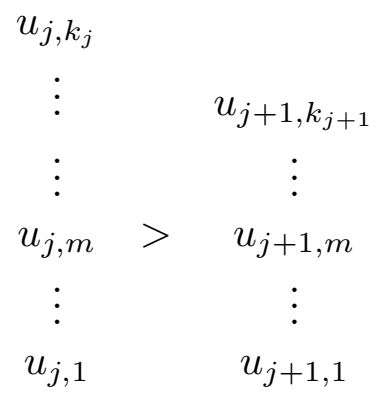


where $u_{j, m}>u_{j+1, m}$. Particularly, we have

$$
u_{j+1,1}<u_{j+1,2}<u_{j+1,3}<\cdots<u_{j+1, m}<u_{j, m}<u_{j, m+1}<\cdots<u_{j, k_{j}} .
$$

Recall that a right transversal of a subgroup $H$ of a group $G$ is a subset $K$ of $G$ consisting of exactly one element from each right coset of $H$ in $G$ (see, for example, [17]). Let $K_{1}$ be the right transversal of

$$
H_{1}=S_{\left\{i_{j+1,1}, i_{j+1,2}, \cdots i_{j+1, m}\right\}} \times S_{\left\{i_{j, m}, i_{j, m+1}, \cdots, i_{j, k_{j}}\right\}}
$$

in the group

$$
G_{1}=S_{\left\{i_{j+1,1}, i_{j+1,2}, \cdots, i_{j+1, m}, i_{j, m}, i_{j, m+1}, \cdots, i_{j, k_{j}}\right\}} \cdot
$$

We may assume that $\epsilon \in K_{1}$, where $\epsilon$ is the identity element of the group $G_{1}$. Now,

$$
\begin{aligned}
& N(I)\left[i_{j+1,1}, i_{j+1,2}, \cdots, i_{j+1, m}, i_{j, m}, \cdots, i_{j, k_{j}}\right]^{\prime}\left(u_{1} \mid v_{1}\right)\left(u_{2} \mid v_{2}\right) \cdots\left(u_{n} \mid v_{n}\right) \\
= & N(I)\left[i_{j+1,1}, i_{j+1,2}, \cdots i_{j+1, m}\right]^{\prime}\left[i_{j, m}, \cdots, i_{j, k_{j}}\right]^{\prime} \sum_{\alpha \in K_{1}} \operatorname{sgn}(\alpha) \alpha\left(u_{1} \mid v_{1}\right)\left(u_{2} \mid v_{2}\right) \cdots\left(u_{n} \mid v_{n}\right) \\
= & m !\left(k_{j}-m+1\right) ! N(I) \sum_{\alpha \in K_{1}} \operatorname{sgn}(\alpha) \alpha\left(u_{1} \mid v_{1}\right)\left(u_{2} \mid v_{2}\right) \cdots\left(u_{n} \mid v_{n}\right) \\
= & m !\left(k_{j}-m+1\right) !(U, V) \operatorname{det}^{\operatorname{det}} \\
+ & m !\left(k_{j}-m+1\right) ! N(I) \sum_{\substack{\alpha \in K_{1} \\
\alpha \neq \epsilon}} \operatorname{sgn}(\alpha) \alpha\left(u_{1} \mid v_{1}\right)\left(u_{2} \mid v_{2}\right) \cdots\left(u_{n} \mid v_{n}\right),
\end{aligned}
$$

For $\alpha \in K_{1}$ and $\alpha \neq \epsilon$, we have that

$$
N(I) \alpha\left(u_{1} \mid v_{1}\right)\left(u_{2} \mid v_{2}\right) \cdots\left(u_{n} \mid v_{n}\right)=\left(P_{\alpha}, V\right)_{d e t}
$$

where $P_{\alpha}$ is a tableau of shape $\lambda$ and $\operatorname{cs}\left(P_{\alpha}\right)<c s(U)$.

Now let $K_{2}$ be the left transversal of

$$
\begin{aligned}
H_{2} & =S_{\left\{i_{j, 1}, i_{j, 2}, \cdots, i_{j, m-1}\right\}} \times S_{\left\{i_{j, m}, i_{j, m+1}, \cdots, i_{j, k_{j}}\right\}} \\
& \times S_{\left\{i_{j+1,1}, i_{j+1,2}, \cdots, i_{j+1, m}\right\}} \times S_{\left\{i_{j+1, m+1}, \cdots, i_{j+1, k_{j+1}}\right\}}
\end{aligned}
$$

in the group

$$
G_{2}=S_{\left\{i_{j, 1}, i_{j, 2}, \cdots, i_{j, k_{j}}\right\}} \times S_{\left\{i_{j+1,1}, i_{j+1,2}, \cdots, i_{j+1, k_{j+1}}\right\}} \cdot
$$

Specifically,

$$
\begin{aligned}
& {\left[i_{j, 1}, i_{j, 2}, \cdots, i_{j, k_{j}}\right]^{\prime}\left[i_{j+1,1}, i_{j+1,2}, \cdots, i_{j+1, k_{j+1}}\right]^{\prime} } \\
= & \sum_{\beta \in K_{2}} \operatorname{sgn}(\beta) \beta\left[i_{j, 1}, i_{j, 2}, \cdots, i_{j, m-1}\right]^{\prime}\left[i_{j, m}, \cdots, i_{j, k_{j}}\right]^{\prime} \\
& {\left[i_{j+1,1}, \cdots, i_{j+1, m}\right]^{\prime}\left[i_{j+1, m+1}, \cdots, i_{j+1, k_{j+1}}\right]^{\prime} . }
\end{aligned}
$$


Therefore,

$$
\begin{aligned}
& N(I)\left[i_{j+1,1}, i_{j+1,2}, \cdots i_{j+1, m}, i_{j, m}, \cdots, i_{j, k_{j}}\right]^{\prime}\left(u_{1} \mid v_{1}\right)\left(u_{2} \mid v_{2}\right) \cdots\left(u_{n} \mid v_{n}\right) \\
= & {\left[i_{1,1}, i_{1,2}, \cdots, i_{1, k_{1}}\right]^{\prime} \cdots\left[i_{j-1,1}, i_{j-1,2}, \cdots, i_{j-1, k_{j-1}}\right]^{\prime} } \\
& {\left[i_{j, 1}, i_{j, 2}, \cdots, i_{j, k_{j}}\right]^{\prime}\left[i_{j+1,1}, i_{j+1,2}, \cdots, i_{j+1, k_{j+1}}\right]^{\prime} } \\
& {\left[i_{j+2,1}, i_{j+2,2}, \cdots, i_{j+2, k_{j+2}}\right]^{\prime} \cdots\left[i_{h, 1}, i_{h, 2}, \cdots, i_{h, k_{h}}\right]^{\prime} } \\
& {\left[i_{j+1,1}, i_{j+1,2}, \cdots, i_{j+1, m}, i_{j, m}, \cdots, i_{j, k_{j}}\right]^{\prime}\left(u_{1} \mid v_{1}\right)\left(u_{2} \mid v_{2}\right) \cdots\left(u_{n} \mid v_{n}\right) } \\
= & {\left[i_{1,1}, i_{1,2}, \cdots, i_{1, k_{1}}\right]^{\prime} \cdots\left[i_{j-1,1}, i_{j-1,2}, \cdots, i_{j-1, k_{j-1}}\right]^{\prime} } \\
& \sum_{\beta \in K_{2}} \operatorname{sgn}(\beta) \beta\left[i_{j, 1}, i_{j, 2}, \cdots, i_{j, m-1}\right]^{\prime}\left[i_{j, m}, \cdots, i_{j, k_{j}}\right]^{\prime}\left[i_{j+1,1}, \cdots, i_{j+1, m}\right]^{\prime} \\
& {\left[i_{j+1, m+1}, \cdots, i_{j+1, k_{j+1}}\right]^{\prime}\left[i_{j+2,1}, i_{j+2,2}, \cdots, i_{j+2, k_{j+2}}\right]^{\prime} \cdots\left[i_{h, 1}, i_{h, 2}, \cdots, i_{h, k_{h}}\right]^{\prime} } \\
& {\left[i_{j+1,1}, i_{j+1,2}, \cdots, i_{j+1, m}, i_{j, m}, \cdots, i_{j, k_{j}}\right]^{\prime} \beta^{-1} \beta\left(u_{1} \mid v_{1}\right)\left(u_{2} \mid v_{2}\right) \cdots\left(u_{n} \mid v_{n}\right) } \\
= & m !\left(k_{j}-m+1\right) !\left[i_{1,1}, i_{1,2}, \cdots, i_{1, k_{1}}\right]^{\prime} \cdots\left[i_{j-1,1}, i_{j-1,2}, \cdots, i_{j-1, k_{j-1}}\right]^{\prime} \\
& \sum_{\beta \in K_{2}}\left[\beta\left(i_{j, 1}\right), \beta\left(i_{j, 2}\right), \cdots, \beta\left(i_{j, m-1}\right)\right]^{\prime}\left[\beta\left(i_{j+1, m+1}\right), \cdots, \beta\left(i_{j+1, k_{j+1}}\right)\right]^{\prime} \\
& {\left[\beta\left(i_{j+2,1}\right), \beta\left(i_{j+2,2}\right), \cdots, \beta\left(i_{j+2, k_{j+2}}\right)\right]^{\prime} \cdots\left[\beta\left(i_{h, 1}\right), \beta\left(i_{h, 2}\right), \cdots, \beta\left(i_{h, k_{h}}\right)\right]^{\prime} } \\
& {\left[\beta\left(i_{j+1,1}\right), \beta\left(i_{j+1,2}\right), \cdots, \beta\left(i_{j+1, m}\right), \beta\left(i_{j, m}\right), \cdots, \beta\left(i_{j, k_{j}}\right)\right]^{\prime} } \\
& s g n(\beta) \beta\left(u_{1} \mid v_{1}\right)\left(u_{2} \mid v_{2}\right) \cdots\left(u_{n} \mid v_{n}\right) \\
= & m !\left(k_{j}-m+1\right) ! \sum_{\beta \in K_{2}} \operatorname{sgn}(\beta)\left(Q_{\beta}, M_{\beta}\right)_{d e t}
\end{aligned}
$$

where the shape of $Q_{\beta}$ and $M_{\beta}$ is higher than the shape of $U$ and $V$. Setting equations (2.8) and (2.9) equal and solving for $(U, V)_{\text {det }}$ yields that $(U, V)_{\text {det }}$ can be written as a linear combination of bideterminants with smaller column sequences or higher shapes. By induction, $(U, V)$ can be written as a linear combination of elements of $\mathcal{C S D}_{n}$ (see equation (2.7)). The proof when $V$ is not column-strict is done in the same manner using equation (2.1). Thus we have the theorem for bideterminants.

The proof for bipermanents is similar. The only major difference between the two proofs is that we need to define the order $<_{s r}$ by setting

$$
(U, V)<_{s r}(M, Q)
$$

where $U$ and $V$ are tableaux of shape $\lambda$ and $M$ and $Q$ are tableaux of shape $\mu$ whenever

1. $\lambda<_{L} \mu$; or

2. if $\lambda=\mu$ then $\operatorname{rs}(U) \operatorname{rs}(V)>_{L} \operatorname{rs}(M) \operatorname{rs}(Q)$.

The following theorems are proven in [7] and [11]. Particularly, they show that both $\mathcal{C S D}$ and $\mathcal{C S P}$ are bases for the letter place algebra. 


\section{Theorem 2.2.}

The collection

$$
\mathcal{C S D}=\left\{(M, Q)_{\text {det }}: M \in \mathcal{C S}, Q \in \mathcal{C S}, \operatorname{sh}(M)=\operatorname{sh}(Q)\right\}
$$

is linearly independent over $\mathbb{C}$.

\section{Theorem 2.3.}

The collection

$$
\mathcal{C S P}=\left\{(M, Q)_{p e r}: M \in \mathcal{C S}, Q \in \mathcal{C S}, \operatorname{sh}(M)=\operatorname{sh}(Q)\right\}
$$

is linearly independent over $\mathbb{C}$.

\section{A Basis for $\mathbb{C}_{S}[X, Y]$.}

Let $\mathcal{L P}_{n}^{*}$ be the subspace of a letter place algebra $\mathcal{L} \mathcal{P}$ that consists of the linear span of the collection

$$
\left\{\left(1 \mid d_{1}\right)\left(2 \mid d_{2}\right) \cdots\left(n \mid d_{n}\right): d_{i} \in \mathcal{A}, 1 \leq i \leq n\right\}
$$

where $d_{i}=\left(d_{i, 1}, d_{i, 2}\right) \in \mathcal{A}$. Define $\phi: \mathcal{L P}_{n}^{*} \rightarrow \mathbb{C}[X, Y]$ by linearly extending the map

$$
\theta\left(\left(1 \mid d_{1}\right)\left(2 \mid d_{2}\right) \cdots\left(n \mid d_{n}\right)\right)=x_{1}^{d_{1,1}} y_{1}^{d_{1,2}} x_{2}^{d_{2,1}} y_{2}^{d_{2,2}} \cdots x_{n}^{d_{n, 1}} y_{n}^{d_{n, 2}}
$$

With $U$ an injective tableau and $V$ a tableau (and $\operatorname{sh}(U)=\operatorname{sh}(V)$ ) with entries from $\mathcal{A}$, we will denote $\phi\left((U, V)_{\text {det }}\right)$ and $\phi\left((U, V)_{\text {per }}\right)$ by $[U, V]_{\text {det }}$ and $[U, V]_{\text {per }}$, respectively. The map $\phi$ is a (vector space) isomorphism. Thus we have

\section{Theorem 3.1.}

The collection

$$
\left\{[U, V]_{\text {det }}: U \in \mathcal{S T}_{n}, V \in \mathcal{C S}_{n}, \operatorname{sh}(U)=\operatorname{sh}(V)\right\}
$$

where $\mathcal{S T}_{n}$ is the collection of all standard tableaux with $n$ cells and $\mathcal{C S}_{n}$ is the collection of all column-strict tableaux with $n$ cells with entries from $\mathcal{A}$, is linearly independent in $\mathbb{C}[X, Y]$.

Let

$$
\psi_{S}=\left[\left[a_{1}, a_{2}, \cdots, a_{j}\right],\left[b_{1}, b_{2}, \cdots, b_{j^{\prime}}\right]\right]
$$

be an ordered pair of two sequences where each of the $a_{i}$ and $b_{i}$ are positive integers and one of the following is true: 
(i) $j \geq 2, j^{\prime} \geq 2, a_{1}=b_{1}=1$ and $j+j^{\prime}=n+1$;

(ii) $j=n, j^{\prime}=0$ and $\psi_{S}=\left[\left[a_{1}, a_{2}, \cdots, a_{n}\right], \emptyset\right]$, where $\emptyset$ denotes the empty sequence;

(iii) $j=0, j^{\prime}=n$ and $\psi_{S}=\left[\emptyset,\left[b_{1}, b_{2}, \cdots, b_{n}\right]\right]$.

For the remainder of this section, $j$ and $j^{\prime}$ will denote the length of $\left[a_{1}, a_{2}, \ldots, a_{j}\right]$ and $\left[b_{1}, b_{2}, \cdots, b_{j^{\prime}}\right]$, respectively. Note that the three possible cases lead to three different possible situations for $\psi_{S}$ :

$$
\begin{gathered}
\psi_{S}=\left[\left[1, a_{2}, \cdots, a_{j}\right],\left[1, b_{2}, \cdots, b_{n-j+1}\right]\right], \\
\psi_{S}=\left[\left[a_{1}, \cdots, a_{n}\right], \emptyset\right]
\end{gathered}
$$

or

$$
\psi_{S}=\left[\emptyset,\left[b_{1}, \cdots, b_{n}\right]\right],
$$

where each of the $a_{i}$ and $b_{i}$ are positive integers. Note that in equation (3.1), we set $a_{1}=b_{1}=1$.

For $1 \leq k \leq j$, let

$$
f_{k}=-1+\sum_{i=1}^{k} a_{i}
$$

and for $1 \leq h \leq j^{\prime}$, let

$$
g_{h}=-1+\sum_{i=1}^{h} b_{i} .
$$

We will say that $\psi_{S}$ is dense if and only if both of the following two conditions hold.

1. For all $k$ such that $1 \leq k \leq j$ and any sequence $\alpha_{k}, \cdots, \alpha_{j}$ of nonnegative integers not all zero, either

$$
f_{k}-\sum_{i=k}^{j} \alpha_{i} a_{i}<0
$$

or

$$
f_{k}-\sum_{i=k}^{j} \alpha_{i} a_{i}=f_{p}
$$

for some $p$.

2. For all $h$ such that $1 \leq h \leq j^{\prime}$ and any sequence $\beta_{h}, \cdots, \beta_{j^{\prime}}$ of nonnegative integers not all zero, either

$$
g_{h}-\sum_{i=h}^{j^{\prime}} \beta_{i} b_{i}<0
$$


or

$$
g_{h}-\sum_{i=h}^{j^{\prime}} \beta_{i} b_{i}=g_{q}
$$

for some $q$.

There are many classes of sequences that are dense. For example,

$$
\psi_{S}=[[1, k, k, k],[1, k, k, k, k]]
$$

for some positive integer $k$. Another such class is

$$
\psi_{S}=\left[\left[1, a_{2}, \cdots, a_{j}\right],\left[1, b_{2}, \cdots, b_{n-j+1}\right]\right]
$$

in which we require $a_{2} \leq a_{3}, a_{i} \mid a_{i+1}$ (for $3 \leq i \leq j-1$ ), $b_{2} \leq b_{3}$ and $b_{i} \mid b_{i+1}$ (for $3 \leq i \leq n-j)$. Another example, would be

$$
\psi_{S}=[[1,1,1,1,3,8],[1,2,2,5,10]]
$$

With $\psi_{S}$ given in (3.1), (3.2) or (3.3), we can construct a subset $S_{\psi}$ of $\mathcal{A}$ in the following manner.

(i) For each $1 \leq i \leq j$, place $\left(0, f_{i}\right)=\left(0,-1+\sum_{k=1}^{i} a_{k}\right)$ in $S_{\psi}$;

(ii) For each $1 \leq i \leq j^{\prime}$, place $\left(g_{i}, 0\right)=\left(-1+\sum_{k=1}^{i} b_{k}, 0\right)$ in $S_{\psi}$.

Equations (3.2) and (3.3) correspond to cases in which $\Delta_{S_{\psi}}(X, Y)$ uses either the variables $Y=\left\{y_{1}, \cdots, y_{n}\right\}$ or $X=\left\{x_{1}, \cdots, x_{n}\right\}$, respectively, but not both. The requirement that $a_{1}=b_{1}=1$ in equation (3.1) comes from the fact that in this case $\Delta_{S_{\psi}}(X, Y)$ uses both sets of variables $X$ and $Y$. Whenever we have $a_{1}=b_{1}=1$, both (i) and (ii) place $(0,0)$ in $S_{\psi}$. Since $S_{\psi}$ is a set (and not a multi-set), the net result is that there is exactly one $(0,0)$ in $S_{\psi}$.

We will say that $S_{\psi}$ is dense if and only if $\psi_{S}$ is dense. Note that given a finite subset $S \subset \mathcal{A}$, it is possible to reverse this process and construct an appropriate $\psi_{S}$.

\section{Examples}

Let $\psi_{S}=[[1,1,1,1,1], \emptyset]$. In this situation,

$$
S_{\psi}=\{(0,4),(0,3),(0,2),(0,1),(0,0)\}
$$


Note that $\Delta_{S_{\psi}}(X, Y)$ is the Vandermonde determinant in the variables $\left\{y_{1}, y_{2}, y_{3}\right.$, $\left.y_{4}, y_{5}\right\}$. With $\psi_{S}=[[1,2,4,4],[1,1,1,3,6]]$,

$$
S_{\psi}=\{(0,10),(0,6),(0,2),(0,0),(1,0),(2,0),(5,0),(11,0)\} .
$$

If $\psi_{S}=[\emptyset,[1,1,2,3,3,3]]$, then

$$
S_{\psi}=\{(0,0),(1,0),(3,0),(6,0),(9,0),(12,0)\} .
$$

If $\psi_{S}=[[2,2,4,4,4], \emptyset]$, then

$$
S_{\psi}=\{(0,15),(0,11),(0,7),(0,3),(0,1)\} .
$$

It is worthwhile to contrast the above collections of $S_{\psi}$ with the set $S$ given in equation (1.12).

Similarly, if

$$
S_{\psi}=\{(0,12),(0,7),(0,2),(0,0),(1,0),(2,0),(5,0),(11,0),(17,0)\}
$$

then $\psi_{S}=[[1,2,5,5],[1,1,1,3,6,6]]$.

With $\psi_{S}$ given in (3.1), (3.2) or (3.3), let $\Upsilon_{\psi_{S}}$ be the collection of all sequences of length $n$

$$
\begin{aligned}
\rho & =\left[h_{j}, h_{j-1}, \cdots, h_{2}, g, e_{2}, \cdots, e_{j^{\prime}}\right] \\
& =\left[\rho_{1}, \cdots, \rho_{j-1}, \rho_{j}, \rho_{j+1}, \cdots, \rho_{n}\right]
\end{aligned}
$$

in which $h_{i}, e_{i}$ and $g$ are nonnegative integers such that $0 \leq h_{i} \leq a_{i}-1$ (for $2 \leq i \leq j$ ), $0 \leq e_{h} \leq b_{h}-1$ (for $\left.2 \leq h \leq j^{\prime}\right)$ and $0 \leq g<a_{1}$ (cases (3.1) or (3.2)) or $0 \leq g<b_{1}$ (case (3.3)). The number of elements $d_{\psi_{S}}=\left|\Upsilon_{\psi_{S}}\right|$ of $\Upsilon_{\psi_{S}}$ is given by

$$
d_{\psi_{S}}=\left|\Upsilon_{\psi_{S}}\right|=\left(\prod_{i=1}^{j} a_{i}\right)\left(\prod_{i=1}^{j^{\prime}} b_{i}\right) \text {. }
$$

With $\psi_{S}$ given in equation (3.1) or equation (3.2), set

$$
\begin{aligned}
\rho^{-} & =\left[a_{j}-1-h_{j}, \cdots, a_{2}-1-h_{2}, a_{1}-1-g, b_{2}-1-e_{2}, \cdots, b_{j^{\prime}}-1-e_{j^{\prime}}\right] \\
& =\left[a_{j}-1-\rho_{1}, \cdots, a_{2}-1-\rho_{j-1}, a_{1}-1-\rho_{j}, b_{2}-1-\rho_{j+1}, \cdots, b_{j^{\prime}}-1-\rho_{n}\right] .
\end{aligned}
$$


The analog for $\rho^{-}$when $\psi_{S}$ is the case found in (3.3) is that we set

$$
\begin{aligned}
\rho^{-} & =\left[b_{1}-1-g, b_{2}-1-e_{2}, \cdots, b_{n}-1-e_{n}\right] \\
& =\left[b_{1}-1-\rho_{1}, b_{2}-1-\rho_{2}, \cdots, b_{n}-1-\rho_{n}\right] .
\end{aligned}
$$

We will say that $i+1$ is northwest of $i$ if $i+1$ is strictly north and weakly west of $i$. Given a standard tableau $V$ of shape $\lambda, \rho=\left[\rho_{1}, \rho_{2}, \cdots, \rho_{j-1}, \rho_{j}, \rho_{j+1}, \cdots, \rho_{n}\right] \in \Upsilon_{\psi_{S}}$ and setting

$$
\delta_{V}(i)=\left\{\begin{array}{ll}
1 & \text { if } i+1 \text { is northwest of } i \text { in } V \\
0 & \text { otherwise }
\end{array},\right.
$$

we define the cocharge tableau $C=C_{\rho}(V)$ of $V$ to be the tableau of shape $\lambda$ with entries from the alphabet $\mathcal{A}$, where

A. i. If $\psi_{S}$ is given in (3.1) (and hence $a_{1}=b_{1}=1$ and $\rho_{j}=0$ ) then place $c_{j}=\left(c_{j, 1}, c_{j, 2}\right)=(0,0)$ in the cell containing $j$ in $V$.

ii. If $\psi_{S}$ is given in (3.2) then place $c_{n}=\left(c_{n, 1}, c_{n, 2}\right)=\left(0, \rho_{n}\right)$ in the cell containing $n$ in $V$.

iii. If $\psi_{S}$ is given in (3.3) then place $c_{1}=\left(c_{1,1}, c_{1,2}\right)=\left(\rho_{1}, 0\right)$ in the cell containing 1 in $V$.

B. Assuming that we have placed $c_{i}=\left(c_{i, 1}, c_{i, 2}\right)$ in the cell containing $i$ in $V$ (for some positive integer $i$ such that $j \leq i<n)$, place $\left(c_{i, 1}+\rho_{i+1}+\delta_{V}(i), 0\right)$ in the cell containing $i+1$ in $V$.

C. Assuming that we have placed $c_{h}=\left(c_{h, 1}, c_{h, 2}\right)$ in the cell containing $h$ in $V$ (for some $2 \leq h \leq j)$, place $\left(0, c_{h, 2}+\rho_{h-1}+\delta_{V}(h-1)\right)$ in the cell containing $h-1$ in $V$.

Given a column-strict tableau $U$ (of shape $\lambda$ ) with entries from $\mathcal{A}$, we can label the entries from smallest to largest (with respect to $<_{\mathcal{A}}$ ) with the integers from 1 to $n$ breaking ties by which entry is the farthest west (left). Let $u_{i}=\left(u_{i, 1}, u_{i, 2}\right)$ be the entry of the cell in $U$ labelled $i$. Note that if $\psi_{S}$ is given by (3.1) and the entry labelled $j, u_{j}$, is not $(0,0)$ then $[I, U]_{\text {per }} \in \mathcal{I}_{S}(X, Y)$. Thus, without loss of generality, we may assume that $u_{j}=(0,0)$ when $\psi_{S}$ is given by equation (3.1). Similarly, with $\psi_{S}$ given in equation (3.2), we may assume that $u_{n} \leq_{\mathcal{A}}(0,0)$. Additionally, with $\psi_{S}$ given by equation (3.3), we may assume that $u_{1} \geq_{\mathcal{A}}(0,0)$. Set $\mathcal{C S}_{S} \subset \mathcal{C S}_{n}$ to be the collection of all $U \in \mathcal{C S}_{n}$ such that

A. If $\psi_{S}$ is given by equation $(3.1)$ then $u_{j}=(0,0)$.

B. If $\psi_{S}$ is given by equation $(3.2)$ then $u_{n} \leq_{\mathcal{A}}(0,0)$.

C. If $\psi_{S}$ is given by equation (3.3) then $u_{1} \geq \mathcal{A}(0,0)$.

Let $U \in \mathcal{C S}_{S}$ and let $V=\operatorname{st}(U)$ be the standard tableau of shape $\lambda$ obtained by placing the label of the cells of $U$ in its respective cell. With $\psi_{S}$ given in (3.1), set

$$
\rho_{i, U}= \begin{cases}\left(u_{i, 2}-u_{i+1,2}-\delta_{V}(i)\right) \quad\left(\bmod a_{j+1-i}\right) & \text { for } 1 \leq i \leq j-1 \\ 0 & i=j ; \\ \left(u_{i, 1}-u_{i-1,1}-\delta_{V}(i-1)\right) \quad\left(\bmod b_{i+j^{\prime}-n}\right) & \text { for } j+1 \leq i \leq n .\end{cases}
$$


Note that we are considering $(\bmod q)$ as a function that maps the integers into

$$
\{0,1, \cdots, q-1\}
$$

and not as a relation.

The analogues for $\rho_{i, U}$ when $\psi_{S}=\left[\left[a_{1}, a_{2}, \cdots, a_{n}\right], \emptyset\right]$ (equation $\left.(3.2)\right)$ or $\psi_{S}=$ $\left[\emptyset,\left[b_{1}, b_{2}, \cdots, b_{n}\right]\right]$ (equation $\left.(3.3)\right)$ is that we set

$$
\rho_{i, U}= \begin{cases}\left(u_{i, 2}-u_{i+1,2}-\delta_{V}(i)\right) & \left(\bmod a_{n+1-i}\right) \\ \left(u_{i, 2}\right) \quad\left(\bmod a_{1}\right) & \text { for } 1 \leq i \leq j-1 \\ i=n\end{cases}
$$

or

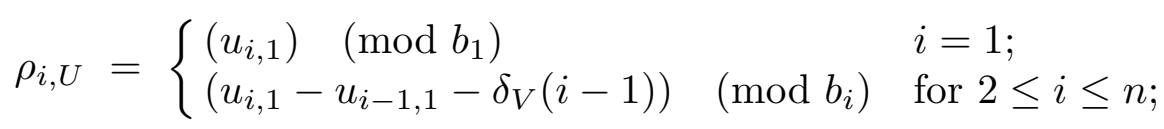

respectively.

Set $\rho_{U}$ to be

$$
\rho_{U}=\left[\rho_{1, U}, \rho_{2, U}, \cdots, \rho_{j-1, U}, \rho_{j, U}, \rho_{j+1, U}, \cdots, \rho_{n, U}\right]
$$

If $U \in \mathcal{C} \mathcal{S}_{S}$ and $C=C_{\rho_{U}}(s t(U))$ and $c_{i}=\left(c_{i, 1}, c_{i, 2}\right)$ is the entry in $C$ that replaced $i$ in $\operatorname{st}(U)$, set

$$
\gamma_{i}= \begin{cases}\left(0, u_{i, 2}-c_{i, 2}\right) & \text { if } \psi_{S}=\left[\left[1, a_{2}, \cdots, a_{j}\right],\left[1, b_{2}, \cdots, b_{n-j+1}\right]\right] \text { and } 1 \leq i \leq j-1 \\ \left(u_{i, 1}-c_{i, 1}, 0\right) & \text { if } \psi_{S}=\left[\left[1, a_{2}, \cdots, a_{j}\right],\left[1, b_{2}, \cdots, b_{n-j+1}\right]\right] \text { and } j+1 \leq i \leq n \\ (0,0) & \text { if } \psi_{S}=\left[\left[1, a_{2}, \cdots, a_{j}\right],\left[1, b_{2}, \cdots, b_{n-j+1}\right]\right] \text { and } i=j ; \\ \left(0, u_{i, 2}-c_{i, 2}\right) & \text { if } \psi_{S}=\left[\left[a_{1}, a_{2}, \cdots, a_{n}\right], \emptyset\right] \text { and } 1 \leq i \leq n \\ \left(u_{i, 1}-c_{i, 1}, 0\right) & \text { if } \psi_{S}=\left[\emptyset,\left[b_{1}, b_{2}, \cdots, b_{n}\right]\right] \text { and } 1 \leq i \leq n\end{cases}
$$

and

$$
\gamma_{\rho_{U}, U}=\left[\gamma_{1}, \gamma_{2}, \cdots, \gamma_{j-1}, \gamma_{j}, \gamma_{j+1}, \cdots, \gamma_{n}\right]
$$

Note that $\rho_{U}$ is a sequence of integers while $\gamma_{\rho_{U}, U}$ is a sequence of elements of $\mathcal{A}$.

\section{Example}

Suppose that

$$
\psi_{S}=[[1,1,3,3,3],[1,2,5,5,5,5]]
$$

and

$$
S_{\psi}=\{(0,10),(0,7),(0,4),(0,1),(0,0),(2,0),(7,0),(12,0),(17,0),(22,0)\} .
$$


Let $U$ be the column-strict tableau

$$
U=\begin{array}{ccccc}
(17,0) & & & & \\
(0,5) & (0,0) & (6,0) & (13,0) & \\
(0,9) & (0,6) & (0,1) & (0,0) & (0,0)
\end{array}
$$

Then the labelling of the entries of $U$ would be

$$
U=\begin{array}{ccccc}
(17,0)_{10} & & & & \\
(0,5)_{3} & (0,0)_{5} & (6,0)_{8} & (13,0)_{9} & \\
(0,9)_{1} & (0,6)_{2} & (0,1)_{4} & (0,0)_{6} & (0,0)_{7}
\end{array}
$$

where the labels are the subscripts to the entries,

$$
\begin{aligned}
& 10 \\
& V=\operatorname{st}(U)=\begin{array}{lllll}
3 & 5 & 8 & 9 & \\
1 & 2 & 4 & 6 & 7
\end{array}, \\
& \rho_{U}=[9-6(\bmod 3), 6-5-1 \quad(\bmod 3), 5-1 \quad(\bmod 3), 1-0-1 \quad(\bmod 1), \\
& 0,0-0(\bmod 2), 0-0(\bmod 5), 6-0-1 \quad(\bmod 5) \text {, } \\
& 13-6(\bmod 5), 17-13-1 \quad(\bmod 5)] \text {, } \\
& =[0,0,1,0,0,0,0,0,2,3] \text {, } \\
& C_{\rho_{U}}(s t(U))=\begin{array}{l}
(7,0) \\
(0,2) \quad(0,0) \quad(1,0) \quad(3,0)
\end{array} \\
& (0,3) \quad(0,3) \quad(0,1) \quad(0,0) \quad(0,0)
\end{aligned}
$$

and

$$
\gamma_{\rho_{U}, U}=[(0,6),(0,3),(0,3),(0,0),(0,0),(0,0),(0,0),(5,0),(10,0),(10,0)]
$$

Note that

$$
\begin{array}{r}
V^{t}=s t(U)^{t}=\begin{array}{rrr}
7 & & \\
6 & 9 & \\
4 & 8 & \\
2 & 5 & \\
1 & 3 & 10
\end{array} \\
\rho_{U}^{-}=[2,2,1,0,0,1,4,4,2,1]
\end{array}
$$

and

$$
\begin{aligned}
& (7,0) \\
& (2,0) \quad(14,0) \\
& C_{\rho_{U}^{-}}\left(s t(U)^{t}\right)=\begin{array}{cc}
(0,0) & (11,0) \\
(0,4) & (0,0)
\end{array} \\
& (0,7) \quad(0,2) \quad(15,0)
\end{aligned}
$$


Note that $(1,0)$ replaced 8 in $s t(U)$ when we constructed $C_{\rho_{U}}(s t(U)) .(11,0)$ replaced 8 in $s t(U)^{t}$ when we constructed $C_{\rho_{U}^{-}}\left(s t(U)^{t}\right)$. Now, $(1,0)+(11,0)=(12,0)$ which is the eighth entry of $S_{\psi}$ as we read from left to right. In fact, the sums for the replacement for $i$ equals the $i^{t h}$ entry of $S_{\psi}$ for $1 \leq i \leq 10$. This leads us to our next lemma.

\section{Lemma 3.2.}

Let $S_{\psi}=\left\{s_{1}, s_{2}, \cdots, s_{n}\right\}, V$ a standard tableau and $\rho \in \Upsilon_{\psi_{S}}$. If $c_{i}$ replaced $i$ in $C=C_{\rho}(V)$ and if $c_{i}^{\prime}$ replaced $i$ in $C^{\prime}=C_{\rho^{-}}\left(V^{t}\right)$ then $c_{i}+c_{i}^{\prime}=s_{i}$.

\section{Proof}

Assume $\psi_{S}$ is of case (3.1) and hence $\rho_{j}=0$. Then $s_{j}=(0,0), c_{j}=(0,0), c_{j}^{\prime}=(0,0)$ and $s_{j}=c_{j}+c_{j}^{\prime}$. Assume for some $i \geq j$ that $c_{i}+c_{i}^{\prime}=s_{i}$. Now,

$$
c_{i+1}=\left(c_{i, 1}+\rho_{i}+\delta_{V}(i), 0\right)
$$

and

$$
c_{i+1}^{\prime}=\left(c_{i, 1}^{\prime}+\rho_{i}^{-}+\delta_{V^{t}}(i), 0\right) .
$$

Note that $\delta_{V}(i)+\delta_{V^{t}}(i)=1, \rho_{i}+\rho_{i}^{-}=b_{i+j^{\prime}-n}-1$ and $c_{i, 1}+c_{i, 1}^{\prime}=s_{i, 1}$. Therefore, we have

$$
\begin{aligned}
c_{i+1}+c_{i+1}^{\prime} & =\left(c_{i, 1}+\rho_{i}+\delta_{V}(i)+c_{i, 1}^{\prime}+\rho_{i}^{-}+\delta_{V^{t}}(i), 0\right) \\
& =\left(s_{i, 1}+b_{i+j^{\prime}-n}, 0\right) \\
& =\left(s_{i+1,1}, 0\right)
\end{aligned}
$$

The other cases are similar.

The following lemma is proven in [4] (see equations (6.5) and (6.6) in Theorem 6.2).

\section{Lemma 3.3.}

Let $S_{\psi}=\left\{s_{1}, s_{2}, \cdots, s_{n}\right\}$, let $Q$ be a standard tableau of shape $\lambda$, let $C$ be a columnstrict tableau with entries from $\mathcal{A}$ also of shape $\lambda$ and let $c_{i}$ be the entry in $C$ that corresponds to the cell containing $i$ in $Q$. Then

$$
[Q, C]_{p e r}\left(\partial_{X}, \partial_{Y}\right) \Delta_{S_{\psi}}(X, Y)=\sum_{\phi \in S_{n}} \operatorname{sgn}(\phi) d_{\phi}\left[Q^{t}, E_{\phi}\right]_{d e t},
$$

where $d_{\phi}$ is an integer and $E_{\phi}$ is the tableau of shape $\lambda$ that has entry $s_{\phi^{-1}(i)}-c_{i}$ in the cell that contains $i$ in $Q^{t}$. Furthermore, if $s_{\phi^{-1}(i)}-c_{i} \notin \mathcal{A}$ for any $1 \leq i \leq n$, then $d_{\phi}=0$; otherwise $d_{\phi}>0$.

\section{Example}

Let $\psi_{S}=[[1,1,4],[1,2]]$. Therefore,

$$
S_{\psi}=\{(0,5),(0,1),(0,0),(2,0)\}
$$


and

$$
\Delta_{S_{\psi}}(X, Y)=\left|\begin{array}{llll}
y_{1}^{5} & y_{1} & 1 & x_{1}^{2} \\
y_{2}^{5} & y_{2} & 1 & x_{2}^{2} \\
y_{3}^{5} & y_{3} & 1 & x_{3}^{2} \\
y_{4}^{5} & y_{4} & 1 & x_{4}^{2}
\end{array}\right|
$$

With $\rho=[2,0,0,1]$ and

$$
M=\begin{array}{lll}
2 & \\
1 & 3 & 4
\end{array}
$$

then

$$
C=C_{\rho}(M)=\begin{aligned}
& (0,0) \\
& (0,3)
\end{aligned}(0,0) \quad(1,0) .
$$

Therefore, ignoring those tableaux with negative entries and letting

$$
Q=\begin{array}{ll}
4 \\
1
\end{array} 23
$$

we have

$$
\begin{aligned}
& {[Q, C]_{\text {per }}\left(\partial_{X}, \partial_{Y}\right) \Delta_{S_{\psi}}(X, Y) } \\
= & 120\left[\begin{array}{rrr}
3 & (1,0) \\
2 & ,(0,0) \\
1 & 4 & (0,2) \\
(0,1)
\end{array}\right]_{\text {det }}+120\left[\begin{array}{rrr}
3 & (1,0) \\
2 & ,(0,1) \\
1 & 4 & (0,2) \\
(0,0)
\end{array}\right]_{\text {det }} .
\end{aligned}
$$

Essentially the coefficient " 120 " in the preceding equation comes from the fact that

$$
\partial_{x_{4}} \partial_{y_{1}}^{3} y_{1}^{5} y_{2} x_{4}^{2}=120 y_{1}^{2} y_{2} x_{4}
$$

Now

$$
\begin{gathered}
M^{t}=\begin{array}{c}
4 \\
3 \\
1
\end{array} \\
\rho^{-}=[1,0,0,0]
\end{gathered}
$$

and

$$
C_{\rho^{-}}\left(M^{t}\right)=\begin{aligned}
& (1,0) \\
& (0,0) \\
& (0,2) \quad(0,1)
\end{aligned} .
$$

Note that $C_{\rho^{-}}\left(M^{t}\right)$ is one of the tableaux that appears in $[Q, C]_{p e r}\left(\partial_{X}, \partial_{Y}\right) \Delta_{S_{\psi}}(X, Y)$. The reason that this occurs in the subject of Theorem 3.4

The type $\tau(W)$ of a tableau $U$ is a listing of the entries of $U$ in decreasing order with respect to $<_{\mathcal{A}}$. For example, with

$$
\begin{array}{llll}
(2,0) & (4,0) & & \\
U= & (0,0) & (3,0) & (3,0) \\
(0,1) & (0,1) & (1,0) & (4,0)
\end{array}
$$


then

$$
\tau(U)=(4,0),(4,0),(3,0),(3,0),(2,0),(1,0),(0,0),(0,1),(0,1) .
$$

Recall that the column sequence of a tableau $U$ is defined to be a listing of the entries

of each column of $U$ from bottom to top starting with the column farthest west and continuing east. For example, the column sequence of $U$ is

$$
c s(U)=(0,1),(0,0),(2,0),(0,1),(3,0),(4,0),(1,0),(3,0),(4,0) .
$$

With $>_{L}$ denoting the lexicographic ordering, we will say that

$$
U<_{s t c} V
$$

when

1. $\operatorname{sh}\left(U^{t}\right)<_{L} \operatorname{sh}\left(V^{t}\right)$ (specifically, $V$ is higher than $U$ ); or

2. if $\operatorname{sh}(U)=\operatorname{sh}(V)$ then $\tau(U)<_{L} \tau(V)$; or

3. if $\operatorname{sh}(U)=\operatorname{sh}(V)$ and $\tau(U)=\tau(V)$ then $c s(U)>_{L} c s(V)$.

Furthermore, we will state that $(U, V)<_{s t c}(P, Q)$ when

1. $U<_{\text {stc }} P$; or

2. if $U=P$ then $V<_{s t c} Q$.

Define

$$
\mathcal{C O}_{S_{\psi}}=\left\{C_{\rho}(V): V \in \mathcal{S} \mathcal{T}_{n} \text { and } \rho \in \Upsilon_{\psi_{S}}\right\}
$$

and

$$
\mathcal{B}_{\psi_{S}}=\left\{[Q, C]_{\text {per }}: Q \in \mathcal{S} \mathcal{T}_{n}, C \in \mathcal{C O}_{S_{\psi}} \text { and } \operatorname{sh}(Q)=\operatorname{sh}(C)\right\}
$$

\section{Theorem 3.4.}

Let $Q$ be a standard tableau of shape $\lambda$ and let $C \in \mathcal{C O}_{S_{\psi}}$ with $\operatorname{sh}(C)=\operatorname{sh}(Q)$. Furthermore, suppose that $C=C_{\rho}(V)$ where $V$ is a standard tableau and $U=C_{\rho^{-}}\left(V^{t}\right)$. Then

$$
[Q, C]_{p e r}\left(\partial_{X}, \partial_{Y}\right) \Delta_{S_{\psi}}(X, Y)=e_{Q^{t}, U}\left[Q^{t}, U\right]_{d e t}+\sum_{\substack{\left(Q^{t}, U\right)<s t c(P, M) \\ P \in \mathcal{S} \mathcal{T}_{n}, M \in \mathcal{C} \mathcal{S}_{n}}} e_{P, M}[P, M]_{\text {det }}
$$

where $e_{Q^{t}, U} \neq 0$. Therefore, the collection $\mathcal{B}_{\psi_{S}}$ is linearly independent in $\mathbb{C}_{S}[X, Y]$.

Proof

Recall that in Theorem 3.1, we showed that the collection

$$
\left\{[M, U]_{\text {det }}: M \in \mathcal{S} \mathcal{T}_{n}, U \in \mathcal{C S}_{n}, \operatorname{sh}(M)=\operatorname{sh}(U)\right\}
$$


is linearly independent in $\mathbb{C}[X, Y]$. Thus equation (3.10) immediately implies that the collection

$$
\left\{[Q, C]_{p e r}\left(\partial_{X}, \partial_{Y}\right) \Delta_{S_{\psi}}(X, Y):[Q, C]_{p e r} \in \mathcal{B}_{\psi_{S}}\right\}
$$

is also a linearly independent set in $\mathbb{C}[X, Y]$. This would imply that the collection $\mathcal{B}_{\psi_{S}}$ is linearly independent in $\mathbb{C}_{S}[X, Y]$ since

$$
\begin{aligned}
& \alpha_{1} p_{1}+\alpha_{2} p_{2}+\cdots+\alpha_{k} p_{k} \in \mathcal{I}_{S}(X, Y) \\
\Leftrightarrow & \left(\alpha_{1} p_{1}+\alpha_{2} p_{2}+\cdots+\alpha_{k} p_{k}\right)\left(\partial_{X}, \partial_{Y}\right) \Delta_{S_{\psi}}(X, Y)=0 \\
\Leftrightarrow & \alpha_{1} p_{1}\left(\partial_{X}, \partial_{Y}\right) \Delta_{S_{\psi}}(X, Y)+\cdots+\alpha_{k} p_{k}\left(\partial_{X}, \partial_{Y}\right) \Delta_{S_{\psi}}(X, Y)=0
\end{aligned}
$$

where $\alpha_{i} \in \mathbb{C}$ and $p_{i} \in \mathcal{B}_{\psi_{S}}$.

Lemma 3.3 implies

$$
[Q, C]_{p e r}\left(\partial_{X}, \partial_{Y}\right) \Delta_{S_{\psi}}(X, Y)=\sum_{\phi \in S_{n}} \operatorname{sgn}(\phi) d_{\phi}\left[Q^{t}, E_{\phi}\right]_{d e t},
$$

where $E_{\phi}$ is the tableau of shape $\lambda$ that has entry $s_{\phi^{-1}(i)}-c_{i}$ in the cell that contains $i$ in $Q^{t}$. Furthermore, if any of the entries $s_{\phi^{-1}(i)}-c_{i} \notin \mathcal{A}$ then $d_{\phi}=0$. Suppose that

$$
[Q, C]_{p e r}=\sum_{\phi \in R(Q)} \phi_{X, Y}\left(x_{1}^{c_{1,1}} x_{2}^{c_{2,1}} \cdots x_{n}^{c_{n, 1}} y_{1}^{c_{1,2}} y_{2}^{c_{2,2}} \cdots y_{n}^{c_{n, 2}}\right)
$$

where $c_{i}=\left(c_{1,1}, c_{1,2}\right)$ is the entry in $C$ that corresponds to the cell containing $i$ in $Q$ and $R(Q)=D\left(Q^{t}\right)$ is defined in equations (2.2) and (2.3). (Recall that the action $\phi_{X, Y}$ is defined in equation (1.5).) Set

$$
q_{Q, C}=x_{1}^{c_{1,1}} x_{2}^{c_{2,1}} \cdots x_{n}^{c_{n, 1}} y_{1}^{c_{1,2}} y_{2}^{c_{2,2}} \cdots y_{n}^{c_{n, 2}}
$$

If $\phi_{X, Y}\left(q_{Q, C}\right)=q_{Q, C}$ and $\phi \in R(Q)=D\left(Q^{t}\right)$ then $c_{i}=c_{\phi(i)}$ for $1 \leq i \leq n$ and

$$
s_{\phi^{-1}(i)}-c_{i}=s_{\phi^{-1}(i)}-c_{\phi^{-1}(i)} \text {. }
$$

Lemma 3.2 implies that

$$
s_{\phi^{-1}(i)}-c_{\phi^{-1}(i)}=u_{\phi^{-1}(i)}
$$

where $u_{i}$ is the $i^{t h}$ entry of $U=C_{\rho^{-}}\left(V^{t}\right)$. Therefore, with $\phi \in R(Q)=D\left(Q^{t}\right)$, we have

$$
\operatorname{sgn}(\phi)\left[Q^{t}, E_{\phi}\right]_{\text {det }}=\operatorname{sgn}(\phi) \operatorname{sgn}(\phi)\left[Q^{t}, U\right]_{\text {det }}=\left[Q^{t}, U\right]_{\text {det }}
$$

For $\phi$ such that $\phi_{X, Y}\left(q_{Q, C}\right) \neq q_{Q, C}$ (i.e., $c_{\phi(i)} \neq c_{i}$ for some $\left.i\right)$, or $\phi \notin R(Q)$, it is not difficult to show that $E_{\phi}>_{s t c} U$ (see, for example, Theorem 6.2 of [4] where it is worked out for the sequence

$$
\psi_{S}=[[1, k, k, \cdots, k],[1, k, \cdots, k]]
$$


in a fashion that is general enough to fit our particular situation).

With

$$
\psi_{S}=\left[\left[1, a_{2}, \cdots, a_{j}\right],\left[1, b_{2}, \cdots, b_{n-j+1}\right]\right]
$$

for $1 \leq t \leq j-1$, set

$$
\gamma_{t}=\left(0, \sum_{i=2}^{j+1-t} \alpha_{i} a_{i}\right)
$$

for $j+1 \leq r \leq n$, set

$$
\gamma_{r}=\left(\sum_{i=2}^{r+j^{\prime}-n} \beta_{i} b_{i}, 0\right)
$$

and set

$$
\Gamma_{\psi_{S}}=\left\{\left(\gamma_{1}, \gamma_{2}, \cdots, \gamma_{j-1},(0,0), \gamma_{j+1}, \cdots, \gamma_{n}\right): \alpha_{2}+\cdots+\alpha_{j}+\beta_{2}+\cdots+\beta_{j^{\prime}}>0\right\}
$$

where $\alpha_{i}$ and $\beta_{i}$ are nonnegative integers. The analogues for $\Gamma_{\psi_{S}}$ when

$$
\psi_{S}=\left[\left[a_{1}, a_{2}, \cdots, a_{n}\right], \emptyset\right]
$$

or

$$
\psi_{S}=\left[\emptyset,\left[b_{1}, b_{2}, \cdots, b_{n}\right]\right]
$$

is that we set

$$
\Gamma_{\psi_{S}}=\left\{\left(\gamma_{1}, \gamma_{2}, \cdots, \gamma_{n}\right): \alpha_{1}+\cdots+\alpha_{n}>0\right\}
$$

with

$$
\gamma_{t}=\left(0, \sum_{i=1}^{n+1-t} \alpha_{i} a_{i}\right)
$$

for $1 \leq t \leq n$, and set

$$
\Gamma_{\psi_{S}}=\left\{\left(\gamma_{1}, \gamma_{2}, \cdots, \gamma_{n}\right): \beta_{1}+\cdots+\beta_{n}>0\right\}
$$

where

$$
\gamma_{r}=\left(\sum_{i=1}^{r} \beta_{i} b_{i}, 0\right)
$$

for $1 \leq r \leq n$, respectively, where the $\alpha_{i}$ and $\beta_{i}$ are nonnegative integers.

With $\gamma=\left(\gamma_{1}, \gamma_{2}, \cdots, \gamma_{n}\right)$ (where $\left.\gamma_{i}=\left(\gamma_{i, 1}, \gamma_{i, 2}\right) \in \mathcal{A}\right)$, set

$$
m_{\gamma}(X, Y)=\sum_{\sigma \in S_{n}} \sigma_{X, Y}\left(x_{1}^{\gamma_{1,1}} y_{1}^{\gamma_{1,2}} x_{2}^{\gamma_{2,1}} y_{2}^{\gamma_{2,2}} \cdots x_{n}^{\gamma_{n, 1}} y_{n}^{\gamma_{n, 2}}\right)
$$


Recall that the action $\sigma_{X, Y}$ is defined in equation (1.5). Note that $m_{\gamma}(X, Y)$ is a symmetric polynomial.

\section{Theorem 3.5.}

If $\psi_{S}$ is dense and $\gamma \in \Gamma_{\psi_{S}}$ then $m_{\gamma}(X, Y) \in \mathcal{I}_{S}(X, Y)$.

\section{Proof}

Assume

$$
\psi_{S}=\left[\left[1, a_{2}, \cdots, a_{j}\right],\left[1, b_{2}, \cdots, b_{n-j+1}\right]\right]
$$

(once again, we have $a_{1}=b_{1}=1$ ). Recall that we construct the subset $S_{\psi}$ of $\mathcal{A}$ in the following manner.

(i) For each $1 \leq h \leq j$, place $\left(0, f_{h}\right)=\left(0,-1+\sum_{k=1}^{h} a_{k}\right)$ in $S_{\psi}$;

(ii) For each $1 \leq i \leq n-j+1$, place $\left(g_{i}, 0\right)=\left(-1+\sum_{k=1}^{i} b_{k}, 0\right)$ in $S_{\psi}$.

Now,

$$
\begin{aligned}
& \Delta_{S_{\psi}}(X, Y) \\
= & \sum_{\beta \in S_{n}} \operatorname{sgn}(\beta) \beta_{X, Y}\left(x_{1}^{0} y_{1}^{f_{j}} x_{2}^{0} y_{2}^{f_{j-1}} \cdots x_{j-1}^{0} y_{j-1}^{f_{2}} x_{j}^{0} y_{j}^{0} x_{j+1}^{g_{2}} y_{j+1}^{0} \cdots x_{n}^{g_{n-j+1}} y_{n}^{0}\right) .
\end{aligned}
$$

If $\gamma=\left(\gamma_{1}, \gamma_{2}, \cdots, \gamma_{n}\right)$ subject to equations (3.11) and (3.12), it is not difficult to show that

$$
\begin{aligned}
& m_{\gamma}\left(\partial_{X}, \partial_{Y}\right) \Delta_{S_{\psi}}(X, Y) \\
= & \sum_{\sigma \in S_{n}} c_{\sigma} \sum_{\beta \in S_{n}} \operatorname{sgn}(\beta) \beta_{X, Y}\left(x_{1}^{0-\gamma_{\sigma(1), 1}} y_{1}^{f_{j}-\gamma_{\sigma(1), 2}} \cdots x_{j-1}^{0-\gamma_{\sigma(j-1), 1}} y_{j-1}^{f_{2}-\gamma_{\sigma(j-1), 2}}\right. \\
& \left.x_{j}^{0-\gamma_{\sigma(j), 1}} y_{j}^{0-\gamma_{\sigma(j), 2}} x_{j+1}^{g_{2,1}-\gamma_{\sigma(j+1), 1}} y_{j+1}^{0-\gamma_{\sigma(j+1), 2}} \cdots x_{n}^{g_{n-j+1,1}-\gamma_{\sigma(n), 1}} y_{n}^{0-\gamma_{\sigma(n), 2}}\right)
\end{aligned}
$$

where $c_{\sigma}=0$ if any of the exponents are negative (see, for example, Theorem 5.2 of [4]).

Suppose $1 \leq h \leq j-1, \sigma(h)=k$ where $j \leq k \leq n$ and $\gamma_{k}=\gamma_{\sigma(h)} \neq(0,0)$ (and, specifically, $\left.\gamma_{\sigma(h), 1}>0\right)$ then the exponent of $x_{h}$ in equation (3.13) is

$$
0-\gamma_{\sigma(h), 1}<0
$$


and therefore $c_{\sigma}=0$. Thus, without loss of generality, we may assume that if $\gamma_{\sigma(h)} \neq$ $(0,0)$ and $1 \leq h \leq j-1$, then $1 \leq \sigma(h) \leq j-1$. Similarly, we may assume that if $\gamma_{\sigma(r)} \neq(0,0)$ and $j+1 \leq r \leq n$, then $j+1 \leq \sigma(r) \leq n$. Let $h$ be the largest integer such that $\gamma_{\sigma(h)} \neq(0,0)$ and $1 \leq h \leq j-1$. Thus for all $q$ such that $h<q \leq j-1$, $\gamma_{\sigma(q)}=(0,0)$. So there are at least $j-h-1(0,0)$ terms in the sequence $\left(\gamma_{1}, \gamma_{2}, \cdots, \gamma_{j-1}\right)$.

Now, $\gamma_{i}<\mathcal{A} \gamma_{i+1}$ yields that

$$
\gamma_{h+1}=\gamma_{h+2}=\cdots=\gamma_{j-1}=(0,0)
$$

Since $\gamma_{k}=\gamma_{\sigma(h)} \neq(0,0)$ we must have $1 \leq k \leq h$. Also, note that

$$
\gamma_{h+1,2}=\gamma_{h+2,2}=\cdots=\gamma_{j-1,2}=0
$$

Recall that for $1 \leq k \leq j-1$ we have

$$
\gamma_{k, 2}=\sum_{i=2}^{j+1-k} \alpha_{i} a_{i}
$$

for some sequence $\left(\alpha_{2}, \alpha_{3}, \cdots, \alpha_{j}\right)$ of nonnegative integers (see equation (3.11)). Thus

$$
\begin{gathered}
0=\gamma_{h+1,2}=\alpha_{2} a_{2}+\cdots+\alpha_{j-h} a_{j-h}, \\
\alpha_{2}=\alpha_{3}=\cdots=\alpha_{j-h}=0
\end{gathered}
$$

and for all $1 \leq k \leq j-1$, we have

$$
\gamma_{k, 2}=\sum_{i=j+1-h}^{j+1-k} \alpha_{i} a_{i}
$$

The exponent of $y_{h}$ in equation (3.13) is, with $k=\sigma(h)$,

$$
\begin{aligned}
f_{j+1-h}-\gamma_{\sigma(h), 2} & =f_{j+1-h}-\gamma_{k, 2} \\
& =f_{j+1-h}-\sum_{i=j+1-h}^{j+1-k} \alpha_{i} a_{i} .
\end{aligned}
$$

With $\gamma_{k, 2} \neq 0$ and $1 \leq k \leq h$, gives us that $j+1-k \geq j+1-h$. Since $\psi_{S}$ is dense, we must have either

$$
f_{j+1-h}-\sum_{i=j+1-h}^{j+1-k} \alpha_{i} a_{i}<0
$$

or

$$
f_{j+1-h}-\sum_{i=j+1-h}^{j+1-k} \alpha_{i} a_{i}=f_{t}
$$


some $t$, where $t<j+1-h$. If the former case, $c_{\sigma}=0$. In the latter, note that $t=j+1-r$ some $r$ where $r>h$. Therefore, $\gamma_{\sigma(r)}=(0,0)$ (recall $h$ is the largest integer $1 \leq h \leq j$ such that $\left.\gamma_{\sigma(r)} \neq(0,0)\right)$. In equation (3.13), the exponent of $y_{r}$ is exactly the same as the exponent of $y_{h}$ (both being exactly $f_{t}$ ) and that the exponent of both $x_{r}$ and $x_{h}$ is 0 . Thus from equation (3.13) we have

$$
\begin{aligned}
& \sum_{\sigma \in S_{n}} c_{\sigma} \sum_{\beta \in S_{n}} \operatorname{sgn}(\beta) \beta_{X, Y}\left(x_{1}^{0-\gamma_{\sigma(1), 1}} y_{1}^{f_{j}-\gamma_{\sigma(1), 2}} \cdots x_{j-1}^{0-\gamma_{\sigma(2), 1}} y_{j-1}^{f_{2}-\gamma_{\sigma(2), 2}}\right. \\
& \left.x_{j}^{0-\gamma_{\sigma(j+1), 1}} y_{j}^{0-\gamma_{\sigma(j+1), 2}} x_{j+1}^{g_{2,1}-\gamma_{\sigma(j+2), 1}} y_{j+1}^{0-\gamma_{\sigma(j+2), 2}} \cdots x_{n}^{g_{n-j+1,1}-\gamma_{\sigma(n), 1}} y_{n}^{0-\gamma_{\sigma(n), 2}}\right) \\
& =0 .
\end{aligned}
$$

(Recall that the action $\beta_{X, Y}$ is defined in equation (1.5).)

The other cases are similar.

In the case that $\psi_{S}=[\emptyset,[1,1,1, \cdots, 1]]$ it is well-known that all symmetric polynomials $m_{\gamma}(X, Y)$ are in the ideal $\mathcal{I}_{S}(X, Y)$. Recall that in this case, $\Delta_{S}(X, Y)$ is the Vandermonde determinant in the variables $X, \mathcal{I}_{S}(X, Y)$ is the ideal generated by the monomials $\left\{y_{1}, y_{2}, \cdots, y_{n}\right\}$ (since $\partial_{y_{i}} \Delta_{S}(X, Y)=0$ ) and the elementary symmetric polynomials in the variables $X$ (see equation (1.12)). This previous theorem informs us that for a dense set $\psi_{S}$ a certain subset of these symmetric polynomials still reside in $\mathcal{I}_{S}(X, Y)$. Specifically,

\section{Corollary 3.6.}

Assume that $\psi_{S}$ is dense. If $U \in \mathcal{C S}_{S}$ and $U \notin \mathcal{C O}_{S_{\psi}}$ then

$$
m_{\gamma_{\rho_{U}, U}}(X, Y) \in \mathcal{I}_{S}(X, Y)
$$

\section{Proof}

Let $V=\operatorname{st}(U)$. Assume

$$
\psi_{S}=\left[\left[1, a_{2}, \cdots, a_{j}\right],\left[1, b_{2}, \cdots, b_{n-j+1}\right]\right]
$$

and

$$
\rho_{U}=\left[\rho_{1}, \rho_{2}, \cdots, \rho_{j-1}, \rho_{j}, \rho_{j+1}, \cdots, \rho_{n}\right] .
$$

Recall that $\gamma_{\rho_{U}, U}$ is defined in equation (3.6). To show that $m_{\gamma_{\rho_{U}, U}} \in \mathcal{I}_{S}(X, Y)$, we need to show that for $1 \leq t \leq j-1$,

$$
\gamma_{t, 2}=\sum_{i=2}^{j+1-t} \alpha_{i} a_{i}
$$


and for $j+1 \leq r \leq n$,

$$
\gamma_{r, 1}=\sum_{i=2}^{r+j^{\prime}-n} \beta_{i} b_{i}
$$

and then apply Theorem 3.5. Since $U \in \mathcal{C S}_{S}$, we may assume that $u_{j}=(0,0)$ and $\gamma_{j}=(0,0)$. Lets assume that for some $k$ such that $j \leq k \leq n$,

$$
\gamma_{k, 1}=\sum_{i=1}^{k+j^{\prime}-n} \beta_{i} b_{i} .
$$

Recall that (see equations (3.5) and (3.6))

$$
\begin{gathered}
\gamma_{k, 1}=u_{k, 1}-c_{k, 1}, \\
\rho_{k+1, U}=\left(u_{k+1,1}-u_{k, 1}-\delta_{V}(k)\right)\left(\bmod b_{k+j^{\prime}-n+1}\right)
\end{gathered}
$$

and

$$
c_{k+1,1}=c_{k, 1}+\rho_{k+1, U}+\delta_{V}(k) .
$$

There exists some integer $\beta_{k+j^{\prime}-n+1} \geq 0$ such that

$$
\begin{aligned}
& \gamma_{k+1,1} \\
= & u_{k+1,1}-c_{k+1,1} \\
= & \beta_{k+j^{\prime}-n+1} b_{k+j^{\prime}-n+1}+u_{k, 1}+\rho_{k+1, U}+\delta_{V}(k)-\left(c_{k, 1}+\rho_{k+1, U}+\delta_{V}(k)\right) \\
= & \beta_{k+j^{\prime}-n+1} b_{k+j^{\prime}-n+1}+u_{k, 1}-c_{k, 1} \\
= & \beta_{k+j^{\prime}-n+1} b_{k+j^{\prime}-n+1}+\gamma_{k, 1} \\
= & \beta_{k+j^{\prime}-n+1} b_{k+j^{\prime}-n+1}+\sum_{i=1}^{k+j^{\prime}-n} \beta_{i} b_{i} \\
= & \sum_{i=1}^{k+j^{\prime}-n+1} \beta_{i} b_{i} .
\end{aligned}
$$

The other cases are similar. $\square$

We will say that

$$
U<_{s t r} V
$$

when

1. $\operatorname{sh}(U)<_{L} \operatorname{sh}(V)$; or

2. if $\operatorname{sh}(U)=\operatorname{sh}(V)$ then $\tau(U)>_{L} \tau(V)$; or

3. if $\operatorname{sh}(U)=\operatorname{sh}(V)$ and $\tau(U)=\tau(V)$ then $r s(U)>_{L} r s(V)$.

Furthermore with $(U, V)$ a pair of tableaux of shape $\mu$ and $(P, Q)$ a pair of tableaux of shape $\nu$, we will say that $(U, V)<_{s t r}(P, Q)$ whenever 
1. $U<_{\text {str } P \text {; or }}$

2. if $U=P$ then $V<_{s t r} Q$.

Let $U$ be a standard tableau and let $R$ be a column-strict tableau of the same shape as $U$. Let $r_{i}$ be the entry in the cell of $R$ that corresponds to the cell containing $i$ in $U$. In [4], the proof of Theorem 5.4 established that

$$
m_{\gamma}(X, Y)[U, R]_{p e r}=\sum_{\alpha \in S_{n}}\left[U, V_{\alpha}\right]_{p e r}
$$

where $\operatorname{sh}\left(V_{\alpha}\right)=\operatorname{sh}(R)$ and $V_{\alpha}$ has entry $r_{i}+\gamma_{\alpha(i)}$ in the cell that corresponds to the cell containing $r_{i}$ in $R$. Note that if $r_{i}+\gamma_{\alpha(i)} \notin \mathcal{A}$, some $i$, then

$$
\left[U, V_{\alpha}\right]_{p e r}\left(\partial_{X}, \partial_{Y}\right) \Delta_{S_{\psi}}(X, Y)=0
$$

and $\left[U, V_{\alpha}\right]_{p e r}(X, Y) \in \mathcal{I}_{S}(X, Y)$.

\section{Example}

Let

$$
\psi_{S}=[[1,1,4],[1,2]]
$$

and

$$
R=\begin{aligned}
& (0,0) \\
& (0,5)
\end{aligned} \quad(0,0) \quad(2,0) .
$$

Note that $R \notin \mathcal{C O}_{S_{\psi}}$. Now,

$$
\begin{aligned}
& \rho_{R}=[0,0,0,0], \\
& s t(R)=\begin{array}{lll}
2 & & \\
1 & 3 & 4
\end{array} \text {, } \\
& C_{\rho}(s t(R))=\begin{array}{l}
(0,0) \\
(0,1) \quad(0,0) \quad(0,0)
\end{array}
\end{aligned}
$$

and

$$
\gamma=\gamma_{\rho_{R}, R}=[(0,4),(0,0),(0,0),(2,0)] .
$$

Now, with

$$
U=\begin{array}{lll}
4 & & \\
1 & 2 & 3
\end{array}
$$

and recalling that

$$
\left[\begin{array}{lll}
U, & (0,0) \\
(0,5) & (0,0) & (2,0)
\end{array}\right]_{p e r}=\left[\begin{array}{lll}
U, & (0,0) \\
(0,5) & (2,0) & (0,0)
\end{array}\right]_{p e r}
$$


(specifically, any row rearrangements of the entries of $R$ does not change the resulting polynomial) yields

$$
\begin{aligned}
& m_{\gamma}(X, Y)\left[U, \begin{array}{lll}
(0,0) & \\
(0,1) & (0,0) & (0,0)
\end{array}\right]_{p e r} \\
& =4\left[\begin{array}{lll}
U,(0,0) \\
(0,5) & (0,0) & (2,0)
\end{array}\right]_{\text {per }}+2\left[\begin{array}{lll}
U, & (2,0) \\
(0,5) & (0,0) & (0,0)
\end{array}\right]_{\text {per }} \\
& +4\left[\begin{array}{lll}
U,(2,0) \\
(0,1) & (0,4) & (0,0)
\end{array}\right]_{\text {per }}+4\left[\begin{array}{lll}
U, & (0,0) \\
(0,1) & (0,4) & (2,0)
\end{array}\right]_{\text {per }} \\
& +4\left[\begin{array}{lll}
U,(0,0) \\
(2,1) & (0,4) & (0,0)
\end{array}\right]_{\text {per }}+4\left[\begin{array}{lll}
U, & (0,4) \\
(0,1) & (0,0) & (2,0)
\end{array}\right]_{\text {per }} \\
& +2\left[U, \begin{array}{lll}
(0,4) & \\
(2,1) & (0,0) & (0,0)
\end{array}\right]_{\text {per }} \text {. }
\end{aligned}
$$

There are some properties of this expansion that need to be considered. First, the polynomial

$$
[U, R]_{\text {per }}=\left[\begin{array}{lll}
U, \begin{array}{l}
(0,0) \\
(0,5)
\end{array}(0,0) & (2,0)
\end{array}\right]_{\text {per }}
$$

is the smallest of all the bipermanents with entries strictly from $\mathcal{A}$ under $<_{s t r}$ with non-zero coefficients. Second, the polynomials

$$
\left[\begin{array}{lll}
U, & (0,4) \\
(2,1) & (0,0) & (0,0)
\end{array}\right]_{\text {per }},\left[\begin{array}{lll}
U, \begin{array}{l}
(0,0) \\
(2,1)
\end{array} & (0,4) & (0,0)
\end{array}\right]_{\text {per }}
$$

are in the ideal $\mathcal{I}_{S}(X, Y)$. Third, since $\psi_{S}$ is dense, $m_{\gamma} \in \mathcal{I}_{S}(X, Y)$ and

$$
m_{\gamma}(X, Y)\left[U, \begin{array}{lll}
(0,0) \\
(0,1) & (0,0) & (0,0)
\end{array}\right]_{p e r} \in \mathcal{I}_{S}(X, Y) .
$$

Thus, in $\mathbb{C}_{S}[X, Y]$ we can solve for $[U, R]_{p e r}$

$$
\begin{aligned}
& {\left[\begin{array}{lll}
U, & (0,0) \\
(0,5) & (0,0) & (2,0)
\end{array}\right]_{p e r}} \\
& \equiv-\frac{1}{2}\left[\begin{array}{lll}
U, \begin{array}{l}
(2,0) \\
(0,5)
\end{array} & (0,0) & (0,0)
\end{array}\right]_{p e r}-\left[\begin{array}{lll}
U, \begin{array}{l}
(2,0) \\
(0,1)
\end{array} & (0,4) & (0,0)
\end{array}\right]_{\text {per }} \\
& -\left[\begin{array}{lll}
U, \begin{array}{l}
(0,0) \\
(0,1)
\end{array} & (0,4) & (2,0)
\end{array}\right]_{p e r}-\left[\begin{array}{lll}
U, \begin{array}{l}
(0,4) \\
(0,1)
\end{array} & (0,0) & (2,0)
\end{array}\right]_{p e r}\left(\bmod \mathcal{I}_{S}(X, Y)\right) \text {. }
\end{aligned}
$$

The previous example is a special case of Theorem 3.7. In [4], the following was essentially proven (see Theorem 5.4 and Corollary 5.5). It should be noted that [4] deals with (using our notation ) the sequence $[[1, k, \cdots, k],[1, k, \cdots, k]]$. 


\section{Theorem 3.7.}

Let $f: \mathcal{C S}_{S} \rightarrow \mathcal{C S} \mathcal{S}_{S}$ be an operator on column-strict tableaux such that if $O=f(V)$ then $\operatorname{sh}(O)=\operatorname{sh}(V)$ and $\operatorname{st}(O)=\operatorname{st}(V)$. Furthermore, suppose that $f$ is such that if $v_{i}$ and $o_{i}$ are the entries in $V$ and $O$, respectively, that are labelled $i$ and that $\gamma_{i}=$ $v_{i}-o_{i} \in \mathcal{A}$ for $1 \leq i \leq n$ then $\gamma_{i} \leq \mathcal{A} \gamma_{i+1}$ for $1 \leq i \leq n-1$. Then, with $\gamma=\left(\gamma_{1}, \cdots, \gamma_{n}\right)$ and $U$ a standard tableau, we have

$$
\begin{aligned}
m_{\gamma}(X, Y)[U, O]_{p e r} & =c_{U, V}[U, V]_{p e r}+\sum_{\substack{(U, V)<s t r(P, Q) \\
P \in \mathcal{S} \mathcal{T}_{n}, Q \in \mathcal{C} \mathcal{S}_{n}}} c_{P, Q}[P, Q]_{p e r} \\
& +\sum_{\substack{P^{\prime} \in \mathcal{S} \mathcal{T}_{n} \\
Q^{\prime} \notin \mathcal{C} \mathcal{S}_{n}}} c_{P^{\prime}, Q^{\prime}}\left[P^{\prime}, Q^{\prime}\right]_{\text {per }}
\end{aligned}
$$

where $c_{U, V} \neq 0$ and each $Q^{\prime}$ is a tableau with at least one entry that is not in $\mathcal{A}$.

Note that there is a slight difference between the order $>_{s t r}$ defined in equation (3.14) and the order $>_{t r}$ defined in [4]. The proof in [4], however, admits the previous theorem.

Note that Theorem 2.1 implies that the collection $\mathcal{C} \mathcal{S}_{S}$ spans $\mathbb{C}_{S}[X, Y]$. (Recall that if $V \in \mathcal{C S}_{n}$ and $V \notin \mathcal{C} \mathcal{S}_{S}$ then $[U, V]_{\text {per }} \in \mathcal{I}_{S}(X, Y)$.) Assume that $V \in \mathcal{C S}_{S}$. Set $f=C_{\rho_{V}}$ and hence $O=C_{\rho_{V}}(V)$. Therefore, in Theorem 3.7, we have $\gamma=\gamma_{\rho_{V}, V}$. Recall that Corollary 3.6 implies that whenever $S$ is dense, $m_{\gamma}(X, Y) \in \mathcal{I}_{S}(X, Y)$. Also, in equation (3.16), since each $Q^{\prime}$ has an entry that is not in the alphabet $\mathcal{A}$, we must have

$$
\left[P^{\prime}, Q^{\prime}\right]_{p e r}\left(\partial_{X}, \partial_{Y}\right) \Delta_{S_{\psi}}(X, Y)=0
$$

and $\left[P^{\prime}, Q^{\prime}\right]_{\text {per }} \in \mathcal{I}_{S}(X, Y)$. Therefore, solving for $[U, V]_{\text {per }}$ in the $\operatorname{ring} \mathbb{C}_{S}[X, Y]$, we have

$$
[U, V]_{\text {per }} \equiv\left(-\frac{1}{c_{U, V}} \sum_{\substack{U, V)<s t r(P, Q) \\ P \in \mathcal{S} \mathcal{T}_{n}, Q \in \mathcal{C} \mathcal{S}_{n}}} c_{P, Q}[P, Q]_{\text {per }}\right) \quad\left(\bmod \mathcal{I}_{S}(X, Y)\right)
$$

It is not difficult to see that if the type of $V$ is either too large or too small, then $[U, V]_{\text {per }} \in \mathcal{I}_{S}(X, Y)$. Therefore, Theorem 3.7 and equation (3.17) imply an algorithm for the expansion of any element of $P(X, Y) \in \mathbb{C}_{S}[X, Y]$ into a linear combination of the collection $\mathcal{B}_{\psi_{S}}$. Thus Theorem 3.4 and Theorem 3.7 imply that

$$
\mathcal{B}_{\psi_{S}}=\left\{[Q, C]_{p e r}: Q \in \mathcal{S} \mathcal{T}_{n}, C \in \mathcal{C O}_{S_{\psi}} \text { and } \operatorname{sh}(Q)=\operatorname{sh}(C)\right\}
$$

is a basis for $\mathbb{C}_{S}[X, Y]$. 
If $R_{u_{1}, u_{2}}$ is the homogeneous subspace of dimension $u_{1}$ in $X$ and dimension $u_{2}$ in $Y$ of a space $R$, then we define the Hilbert series $\mathcal{H}(R)$ to be

$$
\mathcal{H}(R)=\sum_{u_{1}, u_{2}} \operatorname{dim}\left(R_{u_{1}, u_{2}}\right) t^{u_{1}} q^{u_{2}}
$$

We can summarize the above discussion in the following theorem.

\section{Theorem 3.8.}

If $S$ is dense then $\mathcal{B}_{\psi_{S}}$ is a basis for $\mathbb{C}_{S}[X, Y]$ and the Hilbert series $\mathcal{H}\left(\mathbb{C}_{S}[X, Y]\right)$ is given by

$$
\begin{aligned}
\mathcal{H}\left(\mathbb{C}_{S}[X, Y]\right) & =\sum_{\lambda \vdash n} \sum_{(M, N) \in \mathcal{S} \mathcal{T}_{\lambda} \times \mathcal{S} \mathcal{T}_{\lambda}} \sum_{\rho \in \Upsilon_{\psi_{S}}} t^{\left|C_{\rho, 1}(M)\right|} q^{\left|C_{\rho, 2}(M)\right|} \\
& =\sum_{\lambda \vdash n} h_{\lambda} \sum_{M \in \mathcal{S} \mathcal{T}_{\lambda}} \sum_{\rho \in \Upsilon_{\psi_{S}}} t^{\left|C_{\rho, 1}(M)\right|} q^{\left|C_{\rho, 2}(M)\right|}
\end{aligned}
$$

where $\mathcal{S} \mathcal{T}_{\lambda}$ denotes the collection of standard tableaux of shape $\lambda, h_{\lambda}$ denotes the number of standard tableaux of shape $\lambda$ and $\left|C_{\rho, 1}(M)\right|$ and $\left|C_{\rho, 2}(M)\right|$ denote the sum of the first and second coordinates, respectively, of the entries of $C_{\rho}(M)$.

\section{Example}

Let $n=3$ and suppose that

$$
\psi_{S}=[[1,1],[1,2]] .
$$

Therefore,

$$
\Upsilon_{\psi_{S}}=\{[0,0,0],[0,0,1]\} .
$$

Now, letting $N$ denote a standard tableau with three cells and $C_{\rho_{1}}(N)$ and $C_{\rho_{2}}(N)$ the cocharge tableaux with $\rho_{1}=[0,0,0]$ and $\rho_{2}=[0,0,1]$, respectively, we have
$N$
$C_{\rho_{1}}(N)$
$C_{\rho_{2}}(N)$
$1 \quad 2 \quad 3$
$(0,0)$
$(0,0) \quad(0,0)$
$(0,0)$
$(0,0)$

3
2
1
$(1,0)$
$(0,0)$
$(2,0)$
$\begin{array}{ll}2 & \\ 1 & 3\end{array}$
$(0,0)$
$(0,1)$
$(0,0)$
$\begin{array}{ll}3 & \\ 1 & 2\end{array}$
$(1,0)$
$(2,0)$
$(0,0) \quad(0,0)$. 
Recalling that $h_{1^{3}}=1, h_{2,1}=2$ and $h_{3}=1$ yields

$$
\mathcal{H}\left(\mathbb{C}_{S}[X, Y]\right)=1+3 t+3 t q+t^{2} q+2 q+2 t^{2}=(1+t)(1+2 t+2 q+q t) .
$$

The number of pairs $(M, N)$ of standard tableaux with $n$ cells and of the same shape is $n$ !. This immediately implies the following corollary.

\section{Corollary 3.9.}

If $\psi_{S}$ is dense then the dimension (as a vector space) of $\mathbb{C}_{S}[X, Y]$ is $d_{\psi_{S}} n$ ! where

$$
d_{\psi_{S}}=\left(\prod_{k=1}^{j} a_{k}\right)\left(\prod_{k=1}^{j^{\prime}} b_{k}\right) .
$$

It is interesting to note that Theorem 3.4 did not require $S_{\psi}$ to be dense. Thus the collection $\mathcal{B}_{\psi_{S}}$ is linearly independent for any $S_{\psi}$ and $d_{\psi_{S}} n$ ! is a lower bound for the dimension of $\mathbb{C}_{S}[X, Y]$ for any $S_{\psi}$. Note that when $\psi_{S}=[[3,1,1,1], \emptyset]$ (note that this particular $\psi_{S}$ is not dense), in [6] it is shown that the dimension of $\mathbb{C}_{S}[X, Y]$ is 360 . Since $360>3(4 !)$, there do exist rings $\mathbb{C}_{S}[X, Y]$ with dimension larger than $d_{\psi_{S}} n$ !. For a bitableaux bases for $\left.\psi_{S}=[[k, 1,1, \cdots, 1], \emptyset]\right]$ see $[5]$.

Note that with

$$
\rho^{1}=\left(\rho_{1}, \cdots, \rho_{j-2}, \rho_{j-1}, \rho_{j}, \rho_{j+1}, \cdots, \rho_{n}\right)
$$

and

$$
\rho^{2}=\left(\rho_{1}, \cdots, \rho_{j-2}, 0, \rho_{j}, \rho_{j+2}, \cdots, \rho_{n}\right)
$$

(note that we are changing $\rho_{j-1}$ ) that

$$
q^{\left|C_{\rho_{2,2}^{1}}(N)\right|}=\left(q^{j-1}\right)^{\rho_{j-1}} q^{\left|C_{\rho_{2,2}^{2}}(N)\right|} .
$$

Therefore, in equation (3.18), we have

$$
\begin{aligned}
& \sum_{\rho \in \Upsilon_{\psi_{S}}} t^{\left|C_{\rho, 1}(N)\right|} q^{\left|C_{\rho, 2}(N)\right|} \\
= & \left(1+q^{j-1}+\left(q^{j-1}\right)^{2}+\cdots+\left(q^{j-1}\right)^{a_{2}-1}\right) \sum_{\substack{\rho \in \Upsilon_{\psi_{S}} \\
\rho_{j-1}=0}} t^{\left|C_{\rho, 1}(N)\right|} q^{\left|C_{\rho, 2}(N)\right|} \\
= & \left(\frac{1-q^{(j-1) a_{2}}}{1-q^{j-1}}\right) \sum_{\substack{\rho \in \Upsilon_{\psi_{S}} \\
\rho_{j-1}=0}} t^{\left|C_{\rho, 1}(N)\right|} q^{\left|C_{\rho, 2}(N)\right|} .
\end{aligned}
$$


Continuing this argument, let us set $d_{\psi_{S}}(q, t)$ to be

$$
d_{\psi_{S}}(q, t)=\prod_{i=1}^{j}\left(\frac{1-q^{(j+1-i) a_{i}}}{1-q^{j+1-i}}\right) \prod_{i=1}^{j^{\prime}}\left(\frac{1-t^{\left(j^{\prime}+1-i\right) b_{i}}}{1-t^{j^{\prime}+1-i}}\right) .
$$

Therefore, we have

\section{Corollary 3.10.}

Suppose $\psi_{S}$ is dense and let $\Psi=\left[\left[1^{j}\right],\left[1^{j^{\prime}}\right]\right]$. The Hilbert series $\mathcal{H}\left(\mathbb{C}_{S}[X, Y]\right)$ is given by

$$
\mathcal{H}\left(\mathbb{C}_{S}[X, Y]\right)=d_{\psi_{S}}(q, t) \mathcal{H}\left(\mathbb{C}_{S_{\Psi}}[X, Y]\right)
$$

$\phi \in S_{n}$ acting on a basis for $\mathbb{C}_{S}[X, Y]$ yields a representation $\Phi$ of $S_{n}$. With $I$ an injective tableau of shape $\lambda$, let $\phi I$ denote the tableau of shape $\lambda$ obtained by replacing $i$ in $I$ by $\phi(i)$. Let

$$
\mathcal{N}_{\lambda}=\left\{N_{1}, N_{2}, \cdots, N_{h_{\lambda}}\right\}
$$

denote the collection of standard tableaux of shape $\lambda$. Now

$$
\begin{aligned}
\phi\left[N_{i}, U\right]_{\text {per }} & =\left[\phi N_{i}, U\right]_{\text {per }} \\
& =\sum_{k=1}^{h_{\lambda}} e_{i, k}(\phi)\left[N_{k}, U\right]_{p e r}+\sum_{\substack{(P, Q) \in \mathcal{B}_{\psi_{S}} \\
\operatorname{sh}(Q)>_{L} \operatorname{sh}(U)}} g_{P, Q}[P, Q]_{\text {per }} .
\end{aligned}
$$

It is not difficult to show that the matrix $\left(e_{i, k}(\phi)\right)$ is the irreducible $S_{n}$ representation corresponding to shape $\lambda$ (see [1]).

Thus for a given $\rho \in \Upsilon_{\psi_{S}}$, the action of $\phi \in S_{n}$ on the collection

$$
\left\{\left[N_{i}, C_{\rho}\left(N_{k}\right)\right]_{p e r}: N_{i}, N_{k} \in \mathcal{N}_{\lambda}\right\}
$$

yields a representation where the $S_{n}$-irreducible representation of shape $\lambda$ has multiplicity $h_{\lambda}$ (the number of standard tableaux of shape $\lambda$ ). Thus for each $\rho \in \Upsilon_{\psi_{S}}$ there corresponds one copy of the $S_{n}$-regular representation. Therefore, we have

\section{Corollary 3.11.}

If $S$ is dense then the multiplicity of the regular representation of $S_{n}$ in the representation $\Phi$ is $d_{\psi_{S}}$.

Now, let $R_{u_{1}, u_{2}}$ denote the homogeneous component of $\mathbb{C}_{S}[X, Y]$ of degree $u_{1}$ in $X$ and $u_{2}$ in $Y$. With $b \in \mathcal{B}_{\psi_{S}}$ (recall, $\mathcal{B}_{\psi_{S}}$ is a basis for $\mathbb{C}_{S}[X, Y]$ ) and if $b \in R_{u_{1}, u_{2}}$ then 
Theorem 2.1 and Theorem 3.7 imply that $\sigma b \in R_{u_{1}, u_{2}}$. Now, Theorem 3.7 implies that if $p \in R_{u_{1}, u_{2}}$,

$$
p \equiv \sum_{i=1}^{f} d_{i} b_{i} \quad\left(\bmod \mathcal{I}_{S}(X, Y)\right)
$$

and $d_{i} \neq 0$ then $b_{i} \in R_{u_{1}, u_{2}}$. Specifically, if $p \in R_{u_{1}, u_{2}}$, then $\sigma p \in R_{u_{1}, u_{2}}$. Let $\operatorname{char}\left(R_{u_{1}, u_{2}}\right)$ denote the character of the action of $\sigma \in S_{n}$ on $R_{r, s}$. The graded character of $\mathbb{C}_{S}[X, Y]$ is defined as

$$
\operatorname{char}_{q, t}\left(\mathbb{C}_{S}[X, Y]\right)=\sum_{u_{1}, u_{2}} \operatorname{char}\left(R_{r, s}\right) t^{u_{1}} q^{u_{2}} .
$$

\section{Corollary 3.12.}

With $S$ dense, the graded character char ${ }_{q, t}\left(\mathbb{C}_{S}[X, Y]\right)$ of $\mathbb{C}_{S}[X, Y]$ is given by:

$$
\begin{aligned}
\operatorname{char}_{q, t}\left(\mathbb{C}_{S}[X, Y]\right) & =\sum_{\lambda \vdash n} \chi^{\lambda} \sum_{\rho \in \Upsilon_{\psi_{S}}} \sum_{M \in \mathcal{S} \mathcal{T}_{\lambda}} t^{\left|C_{\rho, 1}(M)\right|} q^{\left|C_{\rho, 2}(M)\right|} \\
& =d_{\psi_{S}}(q, t) \sum_{\lambda \vdash n} \chi^{\lambda} \sum_{\substack{M \in \mathcal{S} \mathcal{T}_{\lambda} \\
\rho=\left(0^{j-1}, 0,0^{n-j}\right)}} t^{\left|C_{\rho, 1}(M)\right|} q^{\left|C_{\rho, 2}(M)\right|} \\
& =d_{\psi_{S}}(q, t) \operatorname{char}_{q, t}\left(\mathbb{C}_{S_{\Psi}}[X, Y]\right)
\end{aligned}
$$

where $\mathcal{S} \mathcal{T}_{\lambda}$ denotes the collection of standard tableaux of shape $\lambda$ and $\left|C_{\rho, 1}(M)\right|$ and $\left|C_{\rho, 2}(M)\right|$ denote the sum of the first and second coordinates, respectively, of the entries of $C_{\rho}(M), \chi^{\lambda}$ denotes the irreducible $S_{n}$ character corresponding to shape $\lambda$ and $\Psi=$ $\left[\left[1^{j}\right],\left[1^{j^{\prime}}\right]\right]$.

\section{Example}

With $\psi_{S}$ given in (3.19), we have

$$
\begin{aligned}
\operatorname{char}_{q, t}\left(\mathbb{C}_{S}[X, Y]\right) & =(1+t) \chi^{(3)}+\left(t q+t^{2} q\right) \chi^{(1,1,1)}+\left(q+t q+t+t^{2}\right) \chi^{(2,1)} \\
& =(1+t)\left(\chi^{(3)}+t q \chi^{(1,1,1)}+(t+q) \chi^{(2,1)}\right) .
\end{aligned}
$$

With

$$
\psi_{S}=\left[\emptyset,\left[b_{1}, b_{2}, \cdots, b_{n}\right]\right]
$$

for $1 \leq i \leq n$, set

$$
\lambda_{i}=-i+\sum_{h=1}^{i} b_{h},
$$


and

$$
\lambda=\lambda_{\psi_{S}}=\left(\lambda_{1}, \lambda_{2}, \cdots, \lambda_{n}\right) .
$$

Note that in [6], it is shown that the graded Frobenius characteristic of $\mathbb{C}_{S}[X, Y]$ for any $\psi_{S}$ is given by

$$
\mathcal{F}\left(\mathbb{C}_{S}[X, Y]\right)=\Xi_{\lambda}(t) \tilde{H}_{1^{n}}(x ; q, t)
$$

where $\Xi_{\lambda}(t)$ is the Hilbert series of the graded vector space of skew Schur functions $s_{\lambda / \mu}\left(x_{1}, \cdots, x_{n}\right)$ as $\mu$ varies in $\lambda_{\psi_{S}}$ and $\tilde{H}_{1^{n}}(x ; q, t)$ is a variant of the Macdonald polynomial (see Theorem 2.1 in [6]). Since the Frobenius characteristic of the factor space $\mathbb{C}_{S_{\Psi}}[X, Y]$ where $\Psi=\left[\emptyset,\left[1^{n}\right]\right]$ is $\tilde{H}_{1^{n}}(x ; q, t)$, we get

\section{Corollary 3.13.}

If

$$
\psi_{S}=\left[\emptyset,\left[b_{1}, b_{2}, \cdots, b_{n}\right]\right]
$$

is dense, then

$$
\Xi_{\lambda_{\psi_{S}}}(t)=d_{\psi_{S}}(0, t)
$$

4. The Symmetric Module $\mathbb{C}_{S, T}^{+}[X, Y, Z, W]$.

Let $\mathcal{L P}_{n}^{+}$be the module of polynomials over $\mathbb{C}$ of length $n$ in the indeterminants $\left(\varpi_{i} \mid \varsigma_{k}\right)$ where $\varpi_{i}=\left(\varpi_{i, 1}, \varpi_{i, 2}\right)$ and $\varsigma_{k}=\left(\varsigma_{k, 1}, \varsigma_{k, 2}\right)$ are elements from the alphabet $\mathcal{A}$. Recall that the ring $\mathbb{C}^{S_{n}}[X, Y, Z, W]$ is defined by

$$
\mathbb{C}^{S_{n}}[X, Y, Z, W]=\left\{P(X, Y, Z, W) \in \mathbb{C}[X, Y, Z, W]: \sigma P=P \forall \sigma \in S_{n}\right\} .
$$

Additionally, the ideal $\mathcal{I}_{S, T}^{+}(X, Y, Z, W)$ and the factor space $\mathbb{C}_{S, T}^{+}[X, Y, Z, W]$ are defined by setting

$\mathcal{I}_{S, T}^{+}(X, Y, Z, W)=\left\{P \in \mathbb{C}^{S_{n}}[X, Y, Z, W]: P\left(\partial_{X}, \partial_{Y}, \partial_{Z}, \partial_{W}\right) \Delta_{S}(X, Y) \Delta_{T}(Z, W)=0\right\}$ and

$$
\mathbb{C}_{S, T}^{+}[X, Y, Z, W]=\mathbb{C}^{S_{n}}[X, Y, Z, W] / \mathcal{I}_{S, T}^{+}(X, Y, Z, W) .
$$

Note that the factor space $\mathbb{C}_{S, T}^{+}[X, Y, Z, W]$ is a generalization of the factor spaces found in $[16]$ and [2].

Define the homomorphism $\phi^{+}: \mathcal{L} \mathcal{P}_{n}^{+} \rightarrow \mathbb{C}^{S_{n}}[X, Y, Z, W]$ by linearly extending the map

$$
\begin{aligned}
& \theta^{+}\left(\left(\varpi_{1} \mid \varsigma_{1}\right)\left(\varpi_{2} \mid \varsigma_{2}\right)\left(\varpi_{3} \mid \varsigma_{3}\right) \cdots\left(\varpi_{n} \mid \varsigma_{n}\right)\right) \\
& =\sum_{\sigma \in S_{n}} \sigma_{X, Y, Z, W}\left(x_{1}^{\varpi_{1,1}} x_{2}^{\varpi_{2,1}} \cdots x_{n}^{\varpi_{n, 1}} y_{1}^{\varpi_{1,2}} y_{2}^{\varpi_{2,2}} \cdots y_{n}^{\varpi_{n, 2}} z_{1}^{\varsigma_{1}, 1} \cdots z_{n}^{\varsigma_{n}, 1} w_{1}^{\varsigma_{1}, 2} \cdots w_{n}^{\varsigma_{n, 2}}\right) .
\end{aligned}
$$


Now, let $U$ and $V$ be two column-strict tableaux of shape $\lambda=\left(\lambda_{1}, \lambda_{2}, \cdots, \lambda_{k}\right)$ with entries from $\mathcal{A}$ and let $I$ be an injective tableau of shape $\lambda$. Furthermore, suppose that $u_{i}$ and $v_{i}$ are the entries in the cells of $U$ and $V$ respectively that correspond to the cell containing $i$ in $I$ and let

$$
f_{I}=\lambda_{1} ! \lambda_{2} ! \cdots \lambda_{k} !
$$

Noting that by acting by $\alpha^{-1}$ on both the subscripts and superscripts yields

$$
\begin{aligned}
& x_{1}^{u_{\alpha(1), 1}} x_{2}^{u_{\alpha(2), 1}} \cdots x_{n}^{u_{\alpha(n), 1}} y_{1}^{u_{\alpha(1), 2}} \cdots y_{n}^{u_{\alpha(n), 2}} \\
& =x_{\alpha^{-1}(1)}^{u_{1,1}} x_{\alpha^{-1}(2)}^{u_{2,1}} \cdots x_{\alpha^{-1}(n)}^{u_{n, 1}} \quad y_{\alpha^{-1}(1)}^{u_{1,2}} \cdots y_{\alpha^{-1}(n)}^{u_{n, 2}}
\end{aligned}
$$

and, with $D(I)$ defined in equation (2.3), we have

$$
\begin{aligned}
& \phi^{+}\left((U, V)_{\text {det }}\right) \\
= & \phi^{+}\left(\sum_{\alpha \in D(I)} \operatorname{sgn}(\alpha)\left(u_{\alpha(1)} \mid v_{1}\right)\left(u_{\alpha(2)} \mid v_{2}\right) \cdots\left(u_{\alpha(n)} \mid v_{n}\right)\right) \\
= & \sum_{\sigma \in S_{n}} \sigma_{X, Y, Z, W}\left(\sum_{\alpha \in D(I)} \operatorname{sgn}(\alpha) x_{\alpha(1)}^{u_{1,1}} x_{\alpha(2)}^{u_{2,1}} \cdots x_{\alpha(n)}^{u_{n, 1}} y_{\alpha(1)}^{u_{1,2}} \cdots y_{\alpha(n)}^{u_{n, 2}}\right. \\
& \left.z_{1}^{v_{1,1}} \cdots z_{n}^{v_{n, 1}} w_{1}^{v_{1,2}} \cdots w_{n}^{v_{n, 2}}\right) \\
= & \frac{1}{f_{I^{t}}} \sum_{\sigma \in S_{n}} \sigma_{X, Y, Z, W}\left(\sum _ { \beta \in D ( I ) } \beta _ { X , Y , Z , W } \left(\sum_{\beta^{-1} \alpha \in D(I)} \operatorname{sgn}\left(\beta^{-1}\right) \operatorname{sgn}(\alpha)\right.\right. \\
& x_{\beta^{-1}}^{u_{1,1}} \cdots(1) \\
= & \left.\frac{1}{f_{I^{t}}} \sum_{\sigma \in S_{n}} \sigma_{X, Y, Z, W}^{u_{n, 1}}\left(\sum_{\alpha \in D(n)} y_{\beta^{-1} \alpha(1)}^{u_{1,2}} \operatorname{sgn}(\alpha) y_{\beta^{-1} \alpha(n)}^{u_{n, 2}} x_{\alpha(1)}^{v_{1,1}} \cdots z_{n}^{v_{n, 1}} w_{1}^{v_{1,2}} \cdots x_{\alpha(n)}^{v_{n, 1}} y_{\alpha(1)}^{u_{1,2}} \cdots y_{\alpha(n)}^{u_{n, 2}}\right)\right) \\
& \left.\sum_{\beta \in D(I)} \operatorname{sgn}(\beta) z_{\beta(1)}^{v_{1,1}} \cdots z_{\beta(n)}^{v_{n, 1}} w_{\beta(1)}^{v_{1,2}} \cdots w_{\beta(n)}^{v_{n, 2}}\right) \\
= & \frac{1}{f_{I^{t}}} \sum_{\sigma \in S_{n}} \sigma_{X, Y, Z, W}\left([I, U]_{d e t}(X, Y)[I, V]_{d e t}(Z, W)\right) .
\end{aligned}
$$

Similarly,

$$
\phi^{+}\left((U, V)_{p e r}\right)=\frac{1}{f_{I}} \sum_{\sigma \in S_{n}} \sigma_{X, Y, Z, W}\left([I, U]_{p e r}(X, Y)[I, V]_{p e r}(Z, W)\right) .
$$


We will denote $\phi^{+}\left((U, V)_{d e t}\right)$ and $\phi^{+}\left((U, V)_{p e r}\right)$ by $[U, V]_{d e t}^{+}$and $[U, V]_{p e r}^{+}$, respectively. It is not difficult to see that the definitions of $[V, U]_{\text {per }}^{+}$and $[V, U]_{\text {det }}^{+}$are completely independent of the choice of the injective tableau $I$. Furthermore, note that $\phi^{+}$is a vector space isomorphism between $\mathcal{L P}_{n}^{+}$and $\mathbb{C}^{S_{n}}[X, Y, Z, W]$. Theorem 2.2 and Theorem 2.1, therefore, immediately imply the following two lemmas. (Recall that the collection $\mathcal{C S}_{n}$ consists of column-strict tableaux with entries from the alphabet $\mathcal{A}$.)

\section{Lemma 4.1.}

The collection

$$
\mathcal{G B D}_{n}=\left\{[U, V]_{\text {det }}^{+}: U, V \in C S_{n}, \operatorname{sh}(U)=\operatorname{sh}(V)\right\},
$$

is linearly independent in $\mathbb{C}^{S_{n}}[X, Y, Z, W]$ with coefficients from $\mathbb{C}$.

\section{Lemma 4.2.}

The collection

$$
\mathcal{G B P}_{n}=\left\{[U, V]_{\text {per }}^{+}: U, V \in C S_{n}, \operatorname{sh}(U)=\operatorname{sh}(V)\right\},
$$

spans $\mathbb{C}^{S_{n}}[X, Y, Z, W]$ with coefficients from $\mathbb{C}$.

Recall that with pairs of tableaux $(D, E)$ and $(F, G)$, we have defined

$$
(D, E)<_{s t c}(F, G)
$$

when

1. $D<_{\text {stc }} F$ (see equation (3.7)); or

2. if $D=F$ then $E<_{s t c} G$.

We want to prove that the collection

$$
\mathcal{B S}_{S, T}=\left\{[U, V]_{\text {per }}^{+}: U \in \mathcal{C} \mathcal{O}_{S}, V \in \mathcal{C O}_{T} \text { and } \operatorname{sh}(U)=\operatorname{sh}(V)\right\}
$$

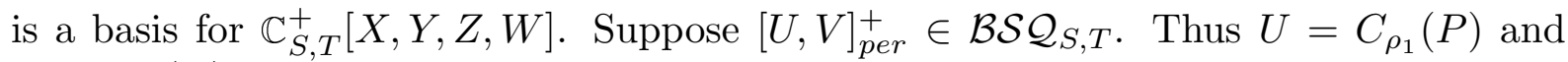
$V=C_{\rho_{2}}(Q)$ for some standard tableaux $P$ and $Q$ and sequences $\rho_{1} \in \Upsilon_{\psi_{S}}$ and $\rho_{2} \in \Upsilon_{\psi_{T}}$. Let $D=C_{\rho_{1}^{-}}(P)$ and $E=C_{\rho_{2}^{-}}(Q)$. Now,

$$
\sigma_{X, Y, Z, W} \Delta_{S}(X, Y) \Delta_{T}(Z, W)=\Delta_{S}(X, Y) \Delta_{T}(Z, W),
$$

(recall equation (1.4)) and thus

$$
\begin{aligned}
& {[U, V]_{p e r}^{+}\left(\partial_{X}, \partial_{Y}, \partial_{Z}, \partial_{W}\right) \Delta_{S}(X, Y) \Delta_{T}(Z, W) } \\
= & \frac{1}{f_{I}} \sum_{\sigma \in S_{n}} \sigma_{X, Y, Z, W}\left([I, U]_{p e r}\left(\partial_{X}, \partial_{Y}\right)[I, V]_{p e r}\left(\partial_{Z}, \partial_{W}\right)\right) \Delta_{S}(X, Y) \Delta_{T}(Z, W) \\
= & \frac{1}{f_{I}} \sum_{\sigma \in S_{n}} \sigma_{X, Y, Z, W}\left([I, U]_{p e r}\left(\partial_{X}, \partial_{Y}\right) \Delta_{S}(X, Y)[I, V]_{p e r}\left(\partial_{Z}, \partial_{W}\right) \Delta_{T}(Z, W)\right) .
\end{aligned}
$$


Lemma 3.3 and Theorem 3.4 imply that

$$
\begin{aligned}
& {[U, V]_{\text {per }}^{+}\left(\partial_{X}, \partial_{Y}, \partial_{Z}, \partial_{W}\right) \Delta_{S}(X, Y) \Delta_{T}(Z, W) } \\
= & \frac{1}{f_{I}} \sum_{\sigma \in S_{n}} \sigma_{X, Y, Z, W}\left([I, U]_{p e r}\left(\partial_{X}, \partial_{Y}\right) \Delta_{S}(X, Y)[I, V]_{p e r}\left(\partial_{Z}, \partial_{W}\right) \Delta_{T}(Z, W)\right) \\
= & \frac{1}{f_{I}} c_{D, E}[D, E]_{\text {det }}^{+}+\sum_{(D, E)<\text { stc }(F, G)} c_{F, G}[F, G]_{d e t}^{+} .
\end{aligned}
$$

where $c_{D, E}>0$. Since the collection $\mathcal{G B D}_{n}$ is linearly independent (Lemma 4.1) and using the fact that

$$
\begin{aligned}
& c_{1} p_{1}+\cdots+c_{m} p_{m} \in \mathcal{I}_{S, T}^{+}(X, Y, Z, W) \\
\Leftrightarrow & \left(c_{1} p_{1}+\cdots+c_{m} p_{m}\right)\left(\partial_{X}, \partial_{Y}, \partial_{Z}, \partial_{W}\right) \Delta_{S}(X, Y) \Delta_{T}(Z, W)=0 \\
\Leftrightarrow & c_{1} p_{1}\left(\partial_{X}, \partial_{Y}, \partial_{Z}, \partial_{W}\right) \Delta_{S}(X, Y) \Delta_{T}(Z, W)+\cdots \\
& +c_{m} p_{m}\left(\partial_{X}, \partial_{Y}, \partial_{Z}, \partial_{W}\right) \Delta_{S}(X, Y) \Delta_{T}(Z, W)=0,
\end{aligned}
$$

where $c_{i} \in \mathbb{C}$ and $p_{i} \in \mathcal{B S} \mathcal{Q}_{S, T}$, we have

\section{Theorem 4.3.}

The collection $\mathcal{B S} \mathcal{Q}_{S, T}$ is linearly independent in $\mathbb{C}_{S, T}^{+}[X, Y, Z, W]$.

Let $(P, Q)$ and $(U, V)$ be pairs of tableaux. Recall that we have defined

$$
(P, Q)<_{s t r}(U, V)
$$

if

1. $P<_{\text {str }} U$ (see equation (3.14)); or

2. if $P=U$ then $Q<_{s t r} V$.

Now suppose that $[U, V]_{\text {per }}^{+} \in \mathcal{G B P}$ but $[U, V]_{\text {per }}^{+} \notin \mathcal{B S} \mathcal{Q}_{S, T}$. Let $\gamma=\gamma_{\rho_{U}, U}(U)$ and $\nu=\gamma_{\rho_{V}, V}(V)$. Since $[U, V]_{\text {per }}^{+} \notin \mathcal{B S} \mathcal{Q}_{S, T}$, we know that at least one of $\gamma$ and $\nu$ is a sequence not made up of entirely $(0,0)^{\prime} s$. Furthermore, let $s t(U)$ and $s t(V)$ be the standard tableaux constructed from the labellings of $U$ and $V$ respectively. We will set $M=C_{\rho_{U}}(s t(U))$ and $N=C_{\rho_{V}}(s t(V))$. Now,

$$
\begin{aligned}
& m_{\gamma}(X, Y) m_{\nu}(Z, W)[M, N]_{p e r}^{+} \\
= & m_{\gamma}(X, Y) m_{\nu}(Z, W) \sum_{\sigma \in S_{n}} \sigma_{X, Y, Z, W}\left([I, M]_{p e r}(X, Y)[I, N]_{p e r}(Z, W)\right) \\
= & \sum_{\sigma \in S_{n}} \sigma_{X, Y, Z, W}\left(m_{\gamma}(X, Y)[I, M]_{p e r}(X, Y) m_{\nu}(Z, W)[I, N]_{p e r}(Z, W)\right) \\
= & \sum_{\sigma \in S_{n}} \sigma_{X, Y, Z, W}\left(\sum_{\alpha \in S_{n}}\left[I, Q_{\alpha}\right]_{p e r}(X, Y) \sum_{\beta \in S_{n}}\left[I, P_{\beta}\right]_{p e r}(Z, W)\right)
\end{aligned}
$$




$$
\begin{aligned}
& =\sum_{\alpha \in S_{n}} \sum_{\beta \in S_{n}} \sum_{\sigma \in S_{n}} \sigma_{X, Y, Z, W}\left(\left[I, Q_{\alpha}\right]_{p e r}(X, Y)\left[I, P_{\beta}\right]_{p e r}(Z, W)\right) \\
& =\sum_{\alpha \in S_{n}} \sum_{\beta \in S_{n}}\left[Q_{\alpha}, P_{\beta}\right]_{p e r}^{+}
\end{aligned}
$$

where $Q_{\alpha}$ and $P_{\beta}$ are as defined in equation (3.15). Thus, using Theorem 3.7 and the straightening algorithm in Theorem 2.1, we have

$$
\begin{aligned}
& m_{\gamma}(X, Y) m_{\nu}(Z, W)[M, N]_{p e r}^{+} \\
\equiv & \left(c_{U, V}[U, V]_{p e r}^{+}+\sum_{\substack{(P, Q)>s t r(U, V) \\
P, Q \in \mathcal{C} \mathcal{S}_{n}}} c_{P, Q}[P, Q]_{p e r}^{+}\right)\left(\bmod \mathcal{I}_{S, T}^{+}(X, Y, Z, W)\right)
\end{aligned}
$$

where $c_{U, V}>0$. Now

$$
m_{\gamma}(X, Y) m_{\nu}(Z, W) \in \mathcal{I}_{S, T}^{+}(X, Y, Z, W)
$$

if both $S$ and $T$ are dense. Thus, equation (4.7) implies an explicit algorithm for expanding polynomials in $\mathbb{C}_{S, T}^{+}[X, Y, Z, W]$ in terms of $\mathcal{B S} \mathcal{Q}_{S, T}$ (with coefficients from $\mathbb{C}$ or $\left.\mathcal{I}_{S, T}^{+}(X, Y, Z, W)\right)$. Using Theorem 4.3 , we now have

\section{Theorem 4.4.}

If $S$ and $T$ are dense then

$$
\mathcal{B S} \mathcal{Q}_{S, T}=\left\{[U, V]_{p e r}^{+}: U \in \mathcal{C O}_{S}, V \in \mathcal{C O}_{T} \text { and } \operatorname{sh}(U)=\operatorname{sh}(V)\right\}
$$

is a basis for $\mathbb{C}_{S, T}^{+}[X, Y, Z, W]$ with coefficients from $\mathbb{C}$.

If $R_{u_{1}, u_{2}, u_{3}, u_{4}}$ is a homogeneous subspace of dimension $u_{1}$ in $X, u_{2}$ in $Y, u_{3}$ in $Z$ and $u_{4}$ in $W$, then we define the Hilbert series $\mathcal{H}(R)$ to be

$$
\mathcal{H}(R)=\sum_{u_{1}, u_{2}, u_{3}, u_{4}} \operatorname{dim}\left(R_{u_{1}, u_{2}, u_{3}, u_{4}}\right) t^{u_{1}} q^{u_{2}} r^{u_{3}} s^{u_{4}}
$$

Recall that $d_{\psi_{S}}(q, t)$ is a polynomial defined in equation (3.21) and that $\Upsilon_{\psi_{S}}$ and $\Upsilon_{\psi_{T}}$ are collections of sequences defined in equation (3.4).

\section{Corollary 4.5.}

If $S$ and $T$ are dense then the Hilbert series $\mathcal{H}\left(\mathbb{C}_{S, T}^{+}[X, Y, Z, W]\right)$ for $\mathbb{C}_{S, T}^{+}[X, Y, Z, W]$ is given by 


$$
\begin{aligned}
& \mathcal{H}\left(\mathbb{C}_{S, T}^{+}[X, Y, Z, W]\right) \\
= & \sum_{\lambda \vdash n} \sum_{\rho \in \Upsilon_{\psi_{S}}} \sum_{\rho^{\prime} \in \Upsilon_{\psi_{T}}} \sum_{(M, N) \in \mathcal{S} \mathcal{T}_{\lambda} \times \mathcal{S} \mathcal{T}_{\lambda}} t^{\left|C_{\rho, 1}(M)\right|} q^{\left|C_{\rho, 2}(M)\right|} r^{\left|C_{\rho^{\prime}, 1}(N)\right|} s^{\left|C_{\rho^{\prime}, 2}(N)\right|} \\
= & d_{\psi_{S}}(q, t) d_{\psi_{T}}(s, r) \sum_{\substack { \lambda \vdash n \\
\begin{subarray}{c}{(M, N) \in \mathcal{S} \mathcal{T}_{\lambda} \times \mathcal{S} \mathcal{T}_{\lambda} \\
\rho=\left(0^{n}\right), \rho^{\prime}=\left(0^{n}\right){ \lambda \vdash n \\
\begin{subarray} { c } { ( M , N ) \in \mathcal { S } \mathcal { T } _ { \lambda } \times \mathcal { S } \mathcal { T } _ { \lambda } \\
\rho = ( 0 ^ { n } ) , \rho ^ { \prime } = ( 0 ^ { n } ) } }\end{subarray}} t^{\left|C_{\rho, 1}(M)\right|} q^{\left|C_{\rho, 2}(M)\right|} r^{\left|C_{\rho^{\prime}, 1}(N)\right|} s^{\left|C_{\rho^{\prime}, 2}(N)\right|},
\end{aligned}
$$

where $\mathcal{S} \mathcal{T}_{\lambda}$ denotes the collection of standard tableaux of shape $\lambda, C_{\rho}(M)$ denotes a cocharge tableau corresponding to a tableau $M$ and a sequence $\rho,\left|C_{\rho, 1}(M)\right|$ and $\left|C_{\rho, 2}(M)\right|$ denote the sums of the first and second coordinates of the cocharge tableau $C_{\rho}(M)$, respectively.

\section{Example}

With $S=T$ given in (3.19) we have

$$
\begin{aligned}
& \mathcal{H}\left(\mathbb{C}_{S, T}^{+}[X, Y, Z, W]\right) \\
= & (1+t)(1+r)+\left(q+t q+t+t^{2}\right)\left(s+r s+r+r^{2}\right)+\left(t q+t^{2} q\right)\left(r s+r^{2} s\right) \\
= & (1+t)(1+q)(1+(t+q)(r+s)+t q r s) .
\end{aligned}
$$

\section{The Skew-Symmetric Module $\mathbb{C}_{S, T}^{-}[X, Y, Z, W]$.}

We will let $\mathcal{L P}_{n}^{-}$denote the letter-place module over the alphabet $\mathcal{A}$ of length $n$ that is diagonally skew-symmetric. Specifically, with $\varpi_{i}, \varsigma_{i} \in \mathcal{A}$, we have that

$$
\left(\varpi_{1} \mid \varsigma_{1}\right)\left(\varpi_{2} \mid \varsigma_{2}\right)\left(\varpi_{3} \mid \varsigma_{3}\right) \cdots\left(\varpi_{n} \mid \varsigma_{n}\right)=0
$$

if $\left(\varpi_{i} \mid \varsigma_{i}\right)=\left(\varpi_{k} \mid \varsigma_{k}\right)$ for $i \neq k$ and that

$$
\left(\varpi_{\sigma_{1}} \mid \varsigma_{\sigma_{1}}\right)\left(\varpi_{\sigma_{2}} \mid \varsigma_{\sigma_{2}}\right) \cdots\left(\varpi_{\sigma_{n}} \mid \varsigma_{\sigma_{n}}\right)=\operatorname{sgn}(\sigma)\left(\varpi_{1} \mid \varsigma_{1}\right)\left(\varpi_{2} \mid \varsigma_{2}\right) \cdots\left(\varpi_{n} \mid \varsigma_{n}\right) .
$$

Note that Theorem 5 on page 28 of [11] implies that the collection

$$
\left\{(U, V)_{d e t}: U \in \mathcal{C S}_{n}, V \in \mathcal{R S}_{n}, \operatorname{sh}(U)=\operatorname{sh}(V)\right\}
$$

is a basis for $\mathcal{L P}_{n}^{-}$(where $\mathcal{C} \mathcal{S}_{n}$ and $\mathcal{R} \mathcal{S}_{n}$ are the collections of column-strict and rowstrict tableaux, respectively, with $n$ cells with entries from the alphabet $\mathcal{A}$.) Recall that

$$
\mathbb{C}^{-}[X, Y, Z, W]=\left\{P \in \mathbb{C}[X, Y, Z, W]: \sigma_{X, Y, Z, W} P=\operatorname{sgn}(\sigma) P \forall \sigma \in S_{n}\right\},
$$


$\mathcal{I}_{S, T}^{-}(X, Y, Z, W)=\left\{P \in \mathbb{C}^{-}[X, Y, Z, W]: P\left(\partial_{X}, \partial_{Y}, \partial_{Z}, \partial_{W}\right) \Delta_{S}(X, Y) \Delta_{T}(Z, W)=0\right\}$ and

$$
\mathbb{C}_{S, T}^{-}[X, Y, Z, W]=\mathbb{C}^{-}[X, Y, Z, W] / \mathcal{I}_{S, T}^{-}(X, Y, Z, W) .
$$

Define $\phi^{-}: \mathcal{L} \mathcal{P}_{n}^{-} \rightarrow \mathbb{C}^{-}[X, Y, Z, W]$ by linearly extending the map

$$
\begin{aligned}
& \theta^{-}\left(\left(\varpi_{1} \mid \varsigma_{1}\right)\left(\varpi_{2} \mid \varsigma_{2}\right)\left(\varpi_{3} \mid \varsigma_{3}\right) \cdots\left(\varpi_{n} \mid \varsigma_{n}\right)\right) \\
= & \sum_{\sigma \in S_{n}} \operatorname{sgn}(\sigma) \sigma_{X, Y, Z, W}\left(x_{1}^{\varpi_{1,1}} \cdots x_{n}^{\varpi_{n, 1}} y_{1}^{\varpi_{1,2}} \cdots y_{n}^{\varpi_{n, 2}} z_{1}^{\varsigma_{1,1}} \cdots z_{n}^{\varsigma_{n, 1}} w_{1}^{\varsigma_{1,2}} \cdots w_{n}^{\varsigma_{n, 2}}\right)
\end{aligned}
$$

where $\varpi_{i}=\left(\varpi_{i, 1}, \varpi_{i, 2}\right) \in \mathcal{A}$ and $\varsigma_{k}=\left(\varsigma_{k, 1}, \varsigma_{k, 2}\right) \in \mathcal{A}$.

Let $G$ be the (group) direct product $G=S_{n} \times S_{n}$ and let $\mathbb{C}[G]$ be the group algebra on $G$ with coefficients from $\mathbb{C}$. The action of $(\alpha, \beta) \in G$ on a polynomial $P(X, Y, Z, W)$ is defined by (recall equation (1.5))

$$
(\alpha, \beta) P(X, Y, Z, W)=\alpha_{X, Y} \beta_{Z, W} P(X, Y, Z, W) .
$$

We will identify $\alpha_{X, Y}=(\alpha, \epsilon), \beta_{Z, W}=(\epsilon, \beta)$ and $\sigma_{X, Y, Z, W}=(\sigma, \sigma)$, where $\epsilon$ is the identity element in the symmetric group $S_{n}$. With $I$ a standard tableau, recall that $D(I)=R\left(I^{t}\right)$ (see equations (2.2) and (2.3)) and that $f_{I^{t}}$ is the order of the group $D(I)$. In the group algebra $\mathbb{C}[G]$,

$$
\begin{aligned}
& \sum_{\sigma \in S_{n}} \operatorname{sgn}(\sigma) \sigma_{X, Y, Z, W}\left[\sum_{\alpha \in D(I)} \operatorname{sgn}(\alpha) \alpha_{X, Y}\right] \\
= & \frac{1}{f_{I^{t}}} \sum_{\sigma \in S_{n}} \operatorname{sgn}(\sigma) \sigma_{X, Y, Z, W}\left[\sum_{\beta \in R\left(I^{t}\right)} \operatorname{sgn}(\beta) \beta_{X, Y, Z, W}\right. \\
& \left.\sum_{\beta^{-1} \alpha \in D(I)} \operatorname{sgn}\left(\beta^{-1}\right) \operatorname{sgn}(\alpha) \beta_{X, Y}^{-1} \alpha_{X, Y}\right] \\
= & \frac{1}{f_{I^{t}}} \sum_{\sigma \in S_{n}} \operatorname{sgn}(\sigma) \sigma_{X, Y, Z, W}\left[\left(\sum_{\alpha \in D(I)} \operatorname{sgn}(\alpha) \alpha_{X, Y}\right)\left(\sum_{\beta \in R\left(I^{t}\right)} \beta_{Z, W}\right)\right] .
\end{aligned}
$$

Similarly,

$$
\begin{aligned}
& \sum_{\sigma \in S_{n}} \operatorname{sgn}(\sigma) \sigma_{X, Y, Z, W}\left[\sum_{\beta \in R\left(I^{t}\right)} \beta_{Z, W}\right] \\
= & \frac{1}{f_{I^{t}}} \sum_{\sigma \in S_{n}} \operatorname{sgn}(\sigma) \sigma_{X, Y, Z, W}\left[\sum_{\alpha \in D(I)} \operatorname{sgn}(\alpha) \alpha_{X, Y, Z, W} \sum_{\alpha^{-1} \beta \in R\left(I^{t}\right)} \alpha_{Z, W}^{-1} \beta_{Z, W}\right] \\
= & \frac{1}{f_{I^{t}}} \sum_{\sigma \in S_{n}} \operatorname{sgn}(\sigma) \sigma_{X, Y, Z, W}\left[\left(\sum_{\alpha \in D(I)} \operatorname{sgn}(\alpha) \alpha_{X, Y}\right)\left(\sum_{\beta \in R\left(I^{t}\right)} \beta_{Z, W}\right)\right] .
\end{aligned}
$$


The equality of equation (5.3) and equation (5.4) yields the following important identity:

$$
\begin{aligned}
& \sum_{\sigma \in S_{n}} \operatorname{sgn}(\sigma) \sigma_{X, Y, Z, W}\left[\sum_{\alpha \in D(I)} \operatorname{sgn}(\alpha) \alpha_{X, Y}\right] \\
= & \sum_{\sigma \in S_{n}} \operatorname{sgn}(\sigma) \sigma_{X, Y, Z, W}\left[\sum_{\beta \in R\left(I^{t}\right)} \beta_{Z, W}\right] \\
= & \frac{1}{f_{I^{t}}} \sum_{\sigma \in S_{n}} \operatorname{sgn}(\sigma) \sigma_{X, Y, Z, W}\left[\left(\sum_{\alpha \in D(I)} \operatorname{sgn}(\alpha) \alpha_{X, Y}\right)\left(\sum_{\beta \in R\left(I^{t}\right)} \beta_{Z, W}\right)\right] .
\end{aligned}
$$

Let $U$ and $M$ be, respectively, a column-strict tableau and a row-strict tableau both of shape $\lambda$. Equation (5.5) implies

$$
\begin{aligned}
& \phi^{-}\left((U, M)_{d e t}\right) \\
= & \sum_{\sigma \in S_{n}} \operatorname{sgn}(\sigma) \sigma_{X, Y, Z, W}\left[\left(\sum_{\alpha \in D(I)} \operatorname{sgn}(\alpha) \alpha_{X, Y} x_{1}^{\left.u_{1,1} \cdots x_{n}^{u_{n, 1}} y_{1}^{u_{1,2}} \cdots y_{n}^{u_{n, 2}}\right)}\right.\right. \\
& \left.z_{1}^{m_{1,1}} \cdots z_{n}^{m_{n, 1}} w_{1}^{m_{1,2}} \cdots w_{n}^{m_{n, 2}}\right] \\
= & \sum_{\sigma \in S_{n}} \operatorname{sgn}(\sigma) \sigma_{X, Y, Z, W}\left[x_{1}^{u_{1,1}} \cdots x_{n}^{u_{n, 1}} y_{1}^{u_{1,2}} \cdots y_{n}^{u_{n, 2}}\right. \\
& \left.\left(\sum_{\beta \in R\left(I^{t}\right)} \beta_{Z, W} z_{1}^{m_{1,1}} \cdots z_{n}^{m_{n, 1}} w_{1}^{m_{1,2}} \cdots w_{n}^{m_{n, 2}}\right)\right] \\
= & \frac{1}{f_{I^{t}}} \sum_{\sigma \in S_{n}} \operatorname{sgn}(\sigma) \sigma_{X, Y, Z, W}\left([I, U]_{\operatorname{det}}(X, Y)\left[I^{t}, M^{t}\right]_{p e r}(Z, W)\right) .
\end{aligned}
$$

Note that since $M$ is row-strict, $M^{t}$ is column-strict. Let us denote $\phi^{-}\left((U, M)_{\text {det }}\right)$ by $[U, M]_{d e t}^{-}$. Set

$$
\mathcal{C S R S}_{n}=\left\{[U, M]_{d e t}^{-}: U \in \mathcal{C S}_{n}, M \in \mathcal{R S}_{n}, \operatorname{sh}(U)=\operatorname{sh}(M)\right\}
$$

Since $\phi_{n}^{-}$is a (vector space) isomorphism (up to sign), we have

\section{Theorem 5.1.}

The collection $\mathcal{C S R S}_{n}$ is linearly independent in $\mathbb{C}^{-}[X, Y, Z, W]$. 
It is important to note that equations (5.7) and (5.8) imply that the straightening algorithm found in Theorem 2.1 can be used in $\mathcal{L P}_{n}^{-}$to write every $[P, Q]_{\text {det }}^{-}$as a linear combination of elements from $\mathcal{C S R S}$. Particularly, we have the following lemma.

\section{Lemma 5.2.}

Suppose that $U$ and $M$ are tableaux such that $\operatorname{sh}(U)=\mu$ and $\operatorname{sh}(M)=\nu$ where $\mu$ and $\nu$ are not necessarily equal. Let $I_{1}$ and $I_{2}$ be injective tableaux of shape $\mu$ and $\nu$ respectively. Let $\lambda$ be the larger (lexicographically) of $\mu^{t}$ and $\nu^{t}$. Then

$$
\sum_{\sigma \in S_{n}} \operatorname{sgn}(\sigma) \sigma_{X, Y, Z, W}\left(\left[I_{1}, U\right]_{\operatorname{det}}(X, Y)\left[I_{2}^{t}, M^{t}\right]_{p e r}(Z, W)\right)=\sum_{s h\left(P^{t}\right) \geq_{L} \lambda^{t}} c_{P, Q}[P, Q]_{d e t}^{-}
$$

where $[P, Q]_{\text {det }}^{-} \in \mathcal{C S R S}_{n}$.

\section{Proof}

Suppose that $\lambda=\mu^{t}$. Then, assuming that

$$
\left[I_{2}^{t}, M^{t}\right]_{p e r}(Z, W)=\sum_{i} c_{i} p_{i}(Z, W)
$$

where each $p_{i}(Z, W)$ is a monomial in $\mathbb{C}[Z, W]$, and using equations (5.7) and (5.9), we have

$$
\begin{aligned}
& \sum_{\sigma \in S_{n}} \operatorname{sgn}(\sigma) \sigma_{X, Y, Z, W}\left[\left(\left[I_{1}, U\right]_{d e t}(X, Y)\left[I_{2}^{t}, M^{t}\right]_{p e r}(Z, W)\right)\right] \\
= & \sum_{\sigma \in S_{n}} \operatorname{sgn}(\sigma) \sigma_{X, Y, Z, W}\left[\left(\sum_{\alpha \in D\left(I_{1}\right)} \operatorname{sgn}(\alpha) \alpha_{X, Y} x_{1}^{u_{1,1}} \cdots x_{n}^{u_{n, 1}} y_{1}^{u_{1,2}} \cdots y_{n}^{u_{n, 2}}\right)\right. \\
& \left.\sum_{i} c_{i} p_{i}(Z, W)\right] \\
= & \sum_{i} c_{i} \sum_{\sigma \in S_{n}} \operatorname{sgn}(\sigma) \sigma_{X, Y, Z, W}\left[\left(\sum_{\alpha \in D\left(I_{1}\right)} \operatorname{sgn}(\alpha) \alpha_{X, Y} x_{1}^{u_{1,1}} \cdots x_{n}^{u_{n, 1}} y_{1}^{u_{1,2}} \cdots y_{n}^{u_{n, 2}}\right)\right. \\
& \left.p_{i}(Z, W)\right] \\
= & \frac{1}{f_{I_{1}^{t}}} \sum_{i} c_{i} \sum_{\sigma \in S_{n}} \operatorname{sgn}(\sigma) \sigma_{X, Y, Z, W}\left[\left[I_{1}, U\right]_{d e t}(X, Y)\left[I_{1}^{t}, Q_{i}\right]_{p e r}(Z, W)\right] .
\end{aligned}
$$

The proof concludes by applying Theorem 2.1.

The proof when $\lambda=\nu^{t}$ is similar. 
With $I$ an injective tableau of shape $\operatorname{sh}(U)=s h(V)$, set

$$
[U, V]_{p e r}^{-}=\frac{1}{f_{I}} \sum_{\sigma \in S_{n}} \operatorname{sgn}(\sigma) \sigma_{X, Y, Z, W}\left[[I, U]_{p e r}(X, Y)[I, V]_{p e r}\left(\partial_{Z}, \partial_{W}\right) \Delta_{T}(Z, W)\right]
$$

where $f_{I}$ is the order of the group $R(I)$. Our goal is to show that the collection

$$
\mathcal{B A}_{S, T}=\left\{[U, V]_{\text {per }}^{-}: U \in \mathcal{C O}_{S}, V \in \mathcal{C O}_{T}, \operatorname{sh}(U)=\operatorname{sh}(V)\right\}
$$

is a basis for $\mathbb{C}_{S, T}^{-}[X, Y, Z, W]$. To this end, we have the following lemma.

\section{Lemma 5.3.}

If the collection $\left\{p_{1}, p_{2}, \cdots, p_{m}\right\}$ is a basis for $\mathbb{C}_{T}[Z, W]$ then the collection

$$
\left\{p_{1}\left(\partial_{Z}, \partial_{W}\right) \Delta_{T}(Z, W), \cdots, p_{m}\left(\partial_{Z}, \partial_{W}\right) \Delta_{T}(Z, W)\right\}
$$

is a basis for $\mathbb{C}_{T}[Z, W]$.

\section{Proof}

Define an inner product $<,>$ on $\mathbb{C}[Z, W]$ by setting

$$
<P, Q>=\left.P\left(\partial_{Z}, \partial_{W}\right) Q\right|_{Z=W=0}
$$

where $\left.\right|_{Z=W=0}$ indicates that we evaluate the resulting polynomial $P\left(\partial_{Z}, \partial_{W}\right) Q$ at

$$
z_{1}=\cdots=z_{n}=w_{1}=\cdots=w_{n}=0
$$

It is clear that for any polynomial $P \in \mathbb{C}[Z, W]$ that

$$
<P, P>\geq 0
$$

and

$$
<P, P>=0
$$

if and only if $P=0$.

Note that $p_{i}\left(\partial_{Z}, \partial_{W}\right) \Delta_{T}(Z, W) \neq 0$ since each $p_{i}$ is an element of a basis for $\mathbb{C}_{T}[Z, W]$. Suppose that

$$
Q=\sum_{k=1}^{n} c_{k} p_{k}\left(\partial_{Z}, \partial_{W}\right) \Delta_{T}(Z, W) \in \mathcal{I}_{T}(Z, W)
$$


where not all of the $c_{j}$ are equal to zero. Since the collection $\left\{p_{1}, p_{2}, \cdots, p_{m}\right\}$ is a basis for $\mathbb{C}_{T}[Z, W]$,

$$
\sum_{k=1}^{n} c_{k} p_{k} \notin \mathcal{I}_{T}(Z, W)
$$

and $Q \neq 0$. Let

$$
P=\sum_{k=1}^{n} c_{k} p_{k}
$$

and thus we have

$$
P\left(\partial_{Z}, \partial_{W}\right) \Delta_{T}(Z, W)=Q
$$

Set

$$
N=Q\left(\partial_{Z}, \partial_{W}\right) \Delta_{T}(Z, W)=0
$$

and hence $\langle P, N\rangle=0$. However,

$$
\begin{aligned}
<P, N> & =\left.P\left(\partial_{Z}, \partial_{W}\right) Q\left(\partial_{Z}, \partial_{W}\right) \Delta_{T}(Z, W)\right|_{Z=W=0} \\
& =\left.Q\left(\partial_{Z}, \partial_{W}\right) P\left(\partial_{Z}, \partial_{W}\right) \Delta_{T}(Z, W)\right|_{Z=W=0} \\
& =<Q, Q> \\
& >0
\end{aligned}
$$

a contradiction. Thus $Q\left(\partial_{Z}, \partial_{W}\right) \Delta_{T}(Z, W) \neq 0, Q \notin \mathcal{I}_{T}(Z, W)$ and the collection

$$
\left\{p_{1}\left(\partial_{Z}, \partial_{W}\right) \Delta_{T}(Z, W), \cdots, p_{m}\left(\partial_{Z}, \partial_{W}\right) \Delta_{T}(Z, W)\right\}
$$

is linearly independent and hence a basis for $\mathbb{C}_{T}[Z, W]$.

It should be noted that Steinberg uses similar ideas in a different setting (see [18]).

Now,

$$
\begin{aligned}
& c_{1} p_{1}+\cdots+c_{m} p_{m} \in \mathcal{I}_{S, T}^{-}(X, Y, Z, W) \\
\Leftrightarrow & \left(c_{1} p_{1}+\cdots+c_{m} p_{m}\right)\left(\partial_{X}, \partial_{Y}, \partial_{Z}, \partial_{W}\right) \Delta_{S}(X, Y) \Delta_{T}(Z, W)=0 \\
\Leftrightarrow & c_{1} p_{1}\left(\partial_{X}, \partial_{Y}, \partial_{Z}, \partial_{W}\right) \Delta_{S}(X, Y) \Delta_{T}(Z, W)+\cdots \\
& \quad+c_{m} p_{m}\left(\partial_{X}, \partial_{Y}, \partial_{Z}, \partial_{W}\right) \Delta_{S_{\psi}}(X, Y) \Delta_{T}(Z, W)=0 .
\end{aligned}
$$

Thus we have shown

\section{Lemma 5.4.}

The collection $\left\{p_{1}, p_{2}, \cdots, p_{m}\right\}$ is linearly independent in $\mathbb{C}_{S, T}^{-}[X, Y, Z, W]$ if and only if the collection

$$
\begin{gathered}
\left\{p_{1}\left(\partial_{X}, \partial_{Y}, \partial_{Z}, \partial_{W}\right) \Delta_{S}(X, Y) \Delta_{T}(Z, W), \cdots,\right. \\
\left.p_{m}\left(\partial_{X}, \partial_{Y}, \partial_{Z}, \partial_{W}\right) \Delta_{S}(X, Y) \Delta_{T}(Z, W)\right\}
\end{gathered}
$$

is linearly independent in $\mathbb{C}[X, Y, Z, W]$. 
This leads us to our next theorem.

\section{Theorem 5.5.}

The collection

$$
\mathcal{B A}_{\mathcal{S}, T}=\left\{[U, V]_{\text {per }}^{-}: U \in \mathcal{C O}_{S}, V \in \mathcal{C O}_{T}, \operatorname{sh}(U)=\operatorname{sh}(V)\right\}
$$

is linearly independent in $\mathbb{C}_{S, T}^{-}[X, Y, Z, W]$.

\section{Proof}

To prove this theorem, we will show that the collection

$$
\begin{aligned}
& \mathcal{B} \mathcal{A} \mathcal{Q D} \mathcal{D}_{S, T} \\
= & \left\{[U, V]_{\text {per }}^{-}\left(\partial_{X}, \partial_{Y}, \partial_{Z}, \partial_{W}\right) \Delta_{S}(X, Y) \Delta_{T}(Z, W):[U, V]_{p e r}^{-} \in \mathcal{B} \mathcal{A} \mathcal{Q}_{S, T}\right\}
\end{aligned}
$$

is linearly independent in $\mathbb{C}[X, Y, Z, W]$ and then apply Lemma 5.4. To prove the independence of the collection $\mathcal{B} \mathcal{A} \mathcal{Q D}_{S, T}$, we will define a linear map

$$
\eta: \mathbb{C}_{S, T}^{-}[X, Y, Z, W] \rightarrow \mathbb{C}[X, Y, Z, W]
$$

and show that the matrix $\left(g_{i, h}\right)$ defined by

$$
\eta\left(\left[U_{i}, V_{i}\right]_{\text {per }}^{-}\left(\partial_{X}, \partial_{Y}, \partial_{Z}, \partial_{W}\right) \Delta_{S}(X, Y) \Delta_{T}(Z, W)\right)=\sum_{h} g_{i, h}\left[U_{h}, V_{h}^{t}\right]_{d e t}^{-}
$$

where

$$
\left\{\left[U_{1}, V_{1}\right]_{\text {per }}^{-},\left[U_{2}, V_{2}\right]_{\text {per }}^{-}, \cdots,\left[U_{m}, V_{m}\right]_{\text {per }}^{-}\right\}
$$

is a listing of the elements of $\mathcal{B} \mathcal{A} \mathcal{Q}_{S, T}$ in increasing order with respect to $<_{\text {str }}$ (see equation (4.6)) and

$$
\left\{\left[U_{1}, V_{1}^{t}\right]_{\text {det }}^{-},\left[U_{2}, V_{2}^{t}\right]_{d e t}^{-}, \cdots,\left[U_{m}, V_{m}^{t}\right]_{d e t}^{-}\right\}
$$

is a subset of $\mathcal{C} \mathcal{S} \mathcal{S}_{n}$ (see equation (5.10) and Theorem 5.1) listed in increasing order with respect to $<_{s t c}$ (see equation (4.4)), has $\operatorname{rank} m=\left|\mathcal{B} \mathcal{A} \mathcal{Q}_{S, T}\right|$. (Note that the polynomials in the former collection are bipermanents and the polynomials in the latter collection are bideterminants.) Specifically, we will show that $\left(g_{i, h}\right)$ is a nonsingular $m \times m$ matrix.

Suppose that $[U, V]_{\text {per }}^{-} \in \mathcal{B} \mathcal{A} \mathcal{Q}_{S, T}$. Now,

$$
[U, V]_{p e r}^{-}\left(\partial_{X}, \partial_{Y}, \partial_{Z}, \partial_{W}\right) \Delta_{S}(X, Y) \Delta_{T}(Z, W)
$$




$$
\begin{aligned}
= & \frac{1}{f_{I}}\left[\sum_{\sigma \in S_{n}} \operatorname{sgn}(\sigma) \sigma_{X, Y, Z, W}\right. \\
& {\left.\left[[I, U]_{p e r}(X, Y)[I, V]_{p e r}\left(\partial_{Z}, \partial_{W}\right) \Delta_{T}(Z, W)\right]\right]\left(\partial_{X}, \partial_{Y}, \partial_{Z}, \partial_{W}\right) \Delta_{S}(X, Y) \Delta_{T}(Z, W) } \\
= & \frac{1}{f_{I}} \sum_{\sigma \in S_{n}} \operatorname{sgn}(\sigma) \sigma_{X, Y, Z, W}\left[\left[[I, U]_{p e r}\left(\partial_{X}, \partial_{Y}\right) \Delta_{S}(X, Y)\right]\right. \\
& {\left.\left[\left[[I, V]_{p e r}\left(\partial_{Z}, \partial_{W}\right) \Delta_{T}(Z, W)\right]\left(\partial_{Z}, \partial_{W}\right) \Delta_{T}(Z, W)\right]\right] }
\end{aligned}
$$

where $I$ is some injective tableau. Now, let

$$
\mathcal{B}_{\psi_{T}}=\left\{\left[O_{1}, V_{1}\right]_{p e r},\left[O_{2}, V_{2}\right]_{p e r}, \cdots,\left[O_{k}, V_{k}\right]_{p e r}\right\}
$$

be a basis for $\mathbb{C}_{T}[Z, W]$ ordered (from smallest to largest) with respect to $<_{s t r}$ (see equation (4.6)). Therefore, the $k \times k$ matrix $\left(c_{i, h}\right)$ defined by

$$
\begin{aligned}
& {\left[\left[O_{i}, V_{i}\right]_{p e r}\left(\partial_{Z}, \partial_{W}\right) \Delta_{T}(Z, W)\right]\left(\partial_{Z}, \partial_{W}\right) \Delta_{T}(Z, W) } \\
= & \sum_{h} c_{i, h}\left[O_{h}, V_{h}\right]_{p e r}(Z, W)+\sum_{\substack{D \in \mathcal{S}_{n} \\
E \in \mathcal{C} \mathcal{S}_{n}, E \notin \mathcal{E} \mathcal{O}_{T}}} \xi_{D, E}[D, E]_{p e r},
\end{aligned}
$$

is unique and nonsingular since both $\mathcal{B}_{\psi_{T}}$ and

$$
\left\{\left[\left[O_{i}, V_{i}\right]_{p e r}\left(\partial_{Z}, \partial_{W}\right) \Delta_{T}(Z, W)\right]\left(\partial_{Z}, \partial_{W}\right) \Delta_{T}(Z, W): 1 \leq i \leq m\right\}
$$

are bases for $\mathbb{C}_{T}[Z, W]$ (apply Lemma 5.3 twice). Note

$$
\sum_{\substack{D \in \mathcal{S} \mathcal{T}_{n} \\ E \in \mathcal{C} \mathcal{S}_{n}, E \notin \mathcal{C} \mathcal{O}_{T}}} \xi_{D, E}[D, E]_{p e r} \in \mathcal{I}_{T}(Z, W)
$$

and $\xi_{D, E} \in \mathbb{C} \cup \mathcal{I}_{T}(Z, W)$.

Some additional properties of equation (5.18) need to be established. Particularly, let $q_{I, T}$ and $r_{O_{i}, V_{i}}$ be monomials such that

$$
\Delta_{T}(Z, W)=\sum_{\alpha \in S_{n}} \operatorname{sgn}(\alpha) \alpha q_{I, T}
$$

and

$$
\left[O_{i}, V_{i}\right]_{p e r}=\sum_{\sigma \in R\left(O_{i}\right)} \sigma r_{O_{i}, V_{i}}
$$


We have (by equation (5.18))

$$
\begin{aligned}
& {\left[\left[O_{i}, V_{i}\right]_{p e r}\left(\partial_{Z}, \partial_{W}\right) \Delta_{T}(Z, W)\right]\left(\partial_{Z}, \partial_{W}\right) \Delta_{T}(Z, W) } \\
= & \sum_{V_{h} \in \mathcal{C} \mathcal{O}_{T}} c_{i, h}\left[O_{h}, V_{h}\right]_{p e r}(Z, W)+\sum_{\substack{D \in \mathcal{S} \mathcal{T}_{n} \\
E \in \mathcal{C} \mathcal{S}_{n}, E \notin \mathcal{C}_{T}}} \xi_{D, E}[D, E]_{p e r} \\
= & {\left[\sum_{\sigma \in R\left(O_{i}\right)} \sigma r_{O_{i}, V_{i}}\right)\left(\partial_{Z}, \partial_{W}\right) } \\
& \left.\left(\operatorname{sgn}(\sigma) \sigma \sum_{\alpha \in S_{n}} \operatorname{sgn}(\alpha) \alpha\left(q_{I, T}\right)\right)\right]\left(\partial_{Z}, \partial_{W}\right)\left(\operatorname{sgn}(\sigma) \sigma \sum_{\beta \in S_{n}} \operatorname{sgn}(\beta) \beta\left(q_{I, T}\right)\right) \\
= & \left.\sum_{\alpha \in S_{n}} \operatorname{sgn}(\alpha) \sum_{\beta \in S_{n}} \operatorname{sgn}(\beta) \sum_{\sigma \in R\left(O_{i}\right)} \sigma\left(\left(r_{O_{i}, V_{i}}\left(\partial_{Z}, \partial_{W}\right) \alpha\left(q_{I, T}\right)\right)\left(\partial_{Z}, \partial_{W}\right)\right) \beta\left(q_{I, T}\right)\right) .
\end{aligned}
$$

Note that the terms

$$
\sum_{\sigma \in R\left(O_{i}\right)} \sigma\left(\left(\left(r_{O_{i}, V_{i}}\left(\partial_{Z}, \partial_{W}\right) \alpha\left(q_{I, T}\right)\right)\left(\partial_{Z}, \partial_{W}\right)\right) \beta\left(q_{I, T}\right)\right)
$$

correspond to bipermanents of shape $\operatorname{sh}\left(O_{i}\right)=\operatorname{sh}\left(V_{i}\right)$ and thus the algorithm implied by Theorem 3.7 gives us that if $\operatorname{sh}\left(V_{h}\right)<_{L} \operatorname{sh}\left(V_{i}\right)$ then $c_{i, h}=0$. Thus $\left(c_{i, h}\right)$ is a upper block triangular matrix and each block is nonsingular (since $\left(c_{i, h}\right)$ is nonsingular). Note also that if $\operatorname{sh}(E)<\operatorname{sh}\left(V_{i}\right)$ then $\xi_{D, E}=0$ (see equation (3.10)).

Additionally, the straightening algorithm associated to Theorem 2.1 yields that

$$
\begin{aligned}
& {\left[\left[O_{i}, V_{i}\right]_{p e r}\left(\partial_{Z}, \partial_{W}\right) \Delta_{T}(Z, W)\right]\left(\partial_{Z}, \partial_{W}\right) \Delta_{T}(Z, W)} \\
& =\sum_{V_{h} \in \mathcal{C O}_{T}} c_{i, h}\left[O_{i}, V_{h}\right]_{p e r}(Z, W)+\sum_{\substack{s h\left(O_{m}\right)>\operatorname{sh}\left(O_{i}\right) \\
V_{m} \in \mathcal{C} \mathcal{O}_{T}, O_{m} \in \mathcal{S} \mathcal{T}_{n}}} c_{i, m}\left[O_{m}, V_{m}\right]_{p e r}(Z, W) \\
& +\sum_{\substack{E \notin \mathcal{C} \mathcal{O}_{T} \\
E \in \mathcal{C} \mathcal{S}_{n}}} \xi_{O_{i}, E}\left[O_{i}, E\right]_{p e r}+\sum_{\substack{D \in \mathcal{S} \mathcal{T}_{n}, s h(D)>>_{L} \operatorname{sh}\left(O_{i}\right) \\
F \notin \mathcal{C} \mathcal{O}_{T}, F \in \mathcal{C} \mathcal{S}_{n}}} \xi_{D, F}[D, F]_{\text {per }} .
\end{aligned}
$$

Now, the coefficients $\left(e_{i}\right)$ defined by

$$
\begin{aligned}
& {\left[O_{i}, U_{i}\right]_{\text {per }}\left(\partial_{X}, \partial_{Y}\right) \Delta_{S}(X, Y) } \\
= & e_{i}\left[O_{i}^{t}, C_{\rho_{U_{i}^{t}}^{-}}\left(U_{i}^{t}\right)\right]_{d e t}+\sum_{\substack{H_{g}>s_{s t c} \rho_{\rho_{U^{t}}^{-}}\left(U_{i}^{t}\right) \\
H_{g} \in \mathcal{C} \mathcal{S}_{n}^{i}}} d_{i, g}\left[O_{i}^{t}, H_{g}\right]_{d e t} \\
+ & \sum_{\substack{s h\left(P^{t}\right)>\operatorname{sh}\left(U_{i}^{t}\right) \\
P \in \mathcal{S} \mathcal{T}_{n}, Q \in \mathcal{C} \mathcal{S}_{n}}} d_{P, Q}[P, Q]_{\text {det }}
\end{aligned}
$$


are nonzero (see Lemma 3.3, Theorem 3.4, equation (3.10) and the algorithm associated to Theorem 2.1). Furthermore, the coefficients $\left(d_{i, g}\right)$ and $\left(d_{P, Q}\right)$ are unique.

Substituting (5.19) and (5.20) into (5.17), setting

$$
\mathcal{V}_{i}=\left\{V_{h} \in \mathcal{C O}_{T}: \operatorname{sh}\left(V_{h}\right)=\operatorname{sh}\left(V_{i}\right)\right\}
$$

$O_{i}=I$ and recalling equation (5.9) and Lemma 5.2 yields

$$
\begin{aligned}
& {\left[U_{i}, V_{i}\right]_{p e r}^{-}\left(\partial_{X}, \partial_{Y}, \partial_{Z}, \partial_{W}\right) \Delta_{S}(X, Y) \Delta_{T}(Z, W)} \\
& =\frac{1}{f_{O_{i}}} \sum_{\sigma \in S_{n}} \operatorname{sgn}(\sigma) \sigma_{X, Y, Z, W}\left[\left[\left[O_{i}, U\right]_{p e r}\left(\partial_{X}, \partial_{Y}\right) \Delta_{S}(X, Y)\right]\right. \\
& \left.\left[\left[\left[O_{i}, V\right]_{p e r}\left(\partial_{Z}, \partial_{W}\right) \Delta_{T}(Z, W)\right]\left(\partial_{Z}, \partial_{W}\right) \Delta_{T}(Z, W)\right]\right] \\
& =\frac{1}{f_{O_{i}}} \sum_{\sigma \in S_{n}} \operatorname{sgn}(\sigma) \sigma_{X, Y, Z, W}\left[\left[e_{i}\left[O_{i}^{t}, C_{\rho_{U_{i}^{t}}^{-}}\left(U_{i}^{t}\right)\right]_{\operatorname{det}}(X, Y)\right.\right. \\
& \left.+\sum_{H_{g}>s_{s t c} C_{\rho^{-}}^{-}\left(U_{i}^{t}\right)} d_{i, g}\left[O_{i}^{t}, H_{g}\right]_{\operatorname{det}}(X, Y)+\sum_{\substack{\operatorname{sh}(P)>\operatorname{sh}\left(U_{i}^{t}\right) \\
P \in \mathcal{S} \mathcal{T}_{n}, Q \in \mathcal{C} \mathcal{S}_{n}}} d_{P, Q}[P, Q]_{\operatorname{det}}(X, Y)\right] \\
& H_{g} \in \mathcal{C S}_{n}^{i} \\
& {\left[\sum_{\substack{s h\left(O_{i}\right)=s h\left(V_{h}\right) \\
V_{h} \in \mathcal{C O} \mathcal{O}_{T}, O_{m} \in \mathcal{S} \mathcal{T}_{n}}} c_{i, h}\left[O_{i}, V_{h}\right]_{p e r}(Z, W)+\sum_{\substack{s h\left(O_{m}\right)>\operatorname{sh}\left(O_{i}\right) \\
V_{m} \in \mathcal{C} \mathcal{O}_{T}}} c_{i, m}\left[O_{m}, V_{m}\right]_{p e r}(Z, W)\right.} \\
& \left.\left.+\sum_{\substack{E \notin \mathcal{C} \mathcal{O}_{T} \\
E \in \mathcal{C} \mathcal{S}_{n}}} \xi_{O_{i}, E}\left[O_{i}, E\right]_{p e r}+\sum_{\substack{D \in \mathcal{S} \mathcal{T}_{n}, \operatorname{sh}(D)>{ }_{L} \operatorname{sh}\left(O_{i}\right) \\
F \notin \mathcal{C} \mathcal{O}_{T}, F \in \mathcal{C} \mathcal{S}_{n}}} \xi_{D, F}[D, F]_{p e r}\right]\right] \\
& =\frac{f_{O_{i}^{t}}}{f_{O_{i}}} \sum_{V_{h} \in \mathcal{V}_{i}} e_{i} c_{i, h}\left[C_{\rho_{U_{i}^{t}}^{-}}\left(U_{i}^{t}\right), V_{h}^{t}\right]_{\text {det }}^{-} \\
& +\sum_{(M, N))>>_{s t c}\left(C_{\left.\rho_{U_{i}^{-}}^{-}\left(U_{i}^{t}\right), V_{h}^{t}\right)}\right.} d_{M, N}[M, N]_{\text {det }}^{-}+\sum_{\substack{Q \notin \mathcal{C} \mathcal{O}_{S} \text { or } \\
E \notin \mathcal{C} \mathcal{O}_{T}}} p_{Q, E}\left[Q, E^{t}\right]_{\text {det }}^{-} \text {, }
\end{aligned}
$$

where $Q$ and $E$ are column-strict tableaux. The coefficients $e_{i}, c_{i, h}$ and $d_{M, N}$ are uniquely defined in equation (5.21).

Recall that $O_{i}$ is a standard tableau of shape $s h\left(U_{i}\right)$. Define

$$
\eta: \mathbb{C}_{S, T}^{-}[X, Y, Z, W] \rightarrow \mathbb{C}[X, Y, Z, W]
$$


by setting

$$
\begin{aligned}
& \eta\left(\left[U_{i}, V_{i}\right]_{p e r}^{-}\left(\partial_{X}, \partial_{Y}, \partial_{Z}, \partial_{W}\right)\right) \\
& =\frac{f_{O_{i}^{t}}}{f_{O_{i}}} \sum_{V_{h} \in \mathcal{V}_{i}} e_{i} c_{i, h}\left[C_{\rho_{U_{i}^{t}}^{-}}\left(U_{i}^{t}\right), V_{h}^{t}\right]_{d e t}^{-}+\sum_{\substack{\left.\left(M, N^{t}\right)\right)>>_{s t c}\left(C_{\rho^{-}}^{-}\left(U_{i}^{t}\right), V_{h}^{t}\right) \\
M \in \mathcal{C} \mathcal{O}_{S}, N \in \mathcal{C} \mathcal{O}_{T}}} d_{M, N}\left[M, N^{t}\right]_{d e t}^{-} \\
& =\sum_{h=1}^{m} g_{i, h}\left[U_{h}, V_{h}^{t}\right]_{d e t}^{-},
\end{aligned}
$$

where the $e_{i}, c_{i, h}$ and $d_{M, N}$ are given in equation (5.21). The matrix $\left(g_{i, h}\right)$ is a upper block triangular matrix where the blocks along the diagonal have the identity

$$
g_{i, h}=e_{i} c_{i, h} \frac{f_{O_{i}^{t}}}{f_{O_{i}}} .
$$

Recall that the matrix $\left(c_{i, h}\right)$ is nonsingular. Thus the rank of $\left(g_{i, h}\right)$ is $m=\left|\mathcal{B} \mathcal{A} \mathcal{Q}_{S, T}\right|$ and $\mathcal{B} \mathcal{A} \mathcal{Q}_{S, T}$ is linearly independent.

We turn our attention to showing that the collection $\mathcal{B} \mathcal{A} \mathcal{Q}_{S, T}$ spans $\mathbb{C}_{S, T}^{-}[X, Y, Z, W]$. We will first show that a collection $\mathcal{C S C O}_{S, T}$ spans $\mathbb{C}_{S, T}^{-}[X, Y, Z, W]$ and then we will show that $\mathcal{B} \mathcal{A} \mathcal{Q}_{S, T}$ spans $\mathcal{C} \mathcal{S C O} \mathcal{O}_{S, T}$. Notice that we have the following equalities concerning $[U, V]_{\text {per }}^{-}$.

$$
\begin{aligned}
& {[U, V]_{p e r}^{-} } \\
= & \frac{1}{f_{I}} \sum_{\sigma \in S_{n}} \operatorname{sgn}(\sigma) \sigma_{X, Y, Z, W}\left[[I, U]_{p e r}(X, Y)[I, V]_{p e r}\left(\partial_{Z}, \partial_{W}\right) \Delta_{T}(Z, W)\right] \\
= & \frac{1}{f_{I}} \sum_{\sigma \in S_{n}} \operatorname{sgn}(\sigma) \sigma_{X, Y, Z, W}\left[\sum_{\alpha \in R(I)} \alpha_{X, Y} x_{1}^{u_{1,1}} \cdots x_{n}^{u_{n, 1}} y_{1}^{u_{1,2}} \cdots y_{n}^{u_{n, 2}}\right. \\
& \left.\sum_{\beta \in R(I)}\left[\beta_{Z, W} \partial_{z_{1}}^{v_{1,1}} \cdots \partial_{z_{n}}^{v_{n, 1}} \partial_{w_{1}}^{v_{1,2}} \cdots \partial_{w_{n}}^{v_{n, 2}}\right] \Delta_{T}(Z, W)\right] \\
= & \frac{1}{f_{I}} \sum_{\sigma \in S_{n}} \operatorname{sgn}(\sigma) \sigma_{X, Y, Z, W}\left[\sum_{\alpha \in R(I)} \alpha_{X, Y} x_{1}^{u_{1,1}} \cdots x_{n}^{u_{n, 1}} y_{1}^{u_{1,2}} \cdots y_{n}^{u_{n, 2}}\right. \\
& \left.\left.\sum_{\alpha \beta R(I)}\left[\alpha, W \beta_{Z, W} \partial_{z_{1}}^{v_{1,1}} \cdots \partial_{z_{n}}^{v_{n, 1}} \partial_{w_{1}}^{v_{1,2}} \cdots \partial_{w_{n}}^{v_{n, 2}}\right)\right]\left[\operatorname{sgn}(\alpha) \alpha_{Z, W} \Delta_{T}(Z, W)\right]\right] \\
= & \frac{1}{f_{I}} \sum_{\sigma \in S_{n}} \operatorname{sgn}(\sigma) \sigma_{X, Y, Z, W}\left[\sum _ { \alpha \in R ( I ) } \operatorname { s g n } ( \alpha ) \alpha _ { X , Y , Z , W } \left[x_{1}^{u_{1,1}} \cdots x_{n}^{u_{n, 1}} y_{1}^{u_{1,2}} \cdots y_{n}^{u_{n, 2}}\right.\right.
\end{aligned}
$$




$$
\begin{aligned}
& \left.\left.\sum_{\beta \in R(I)} \beta_{Z, W} \partial_{z_{1}}^{v_{1,1}} \cdots \partial_{z_{n}}^{v_{n, 1}} \partial_{w_{1}}^{v_{1,2}} \cdots \partial_{w_{n}}^{v_{n, 2}} \Delta_{T}(Z, W)\right]\right] \\
= & \sum_{\sigma \in S_{n}} \operatorname{sgn}(\sigma) \sigma_{X, Y, Z, W}\left[x_{1}^{u_{1,1}} \cdots x_{n}^{u_{n, 1}} y_{1}^{u_{1,2}} \cdots y_{n}^{u_{n, 2}}\right. \\
& \left.\sum_{\beta \in R(I)} \beta_{Z, W} \partial_{z_{1}}^{v_{1,1}} \cdots \partial_{z_{n}}^{v_{n, 1}} \partial_{w_{1}}^{v_{1,2}} \cdots \partial_{w_{n}}^{v_{n, 2}} \Delta_{T}(Z, W)\right] .
\end{aligned}
$$

Similarly, we have that

$$
\begin{aligned}
{[U, V]_{\text {per }}^{-}=} & \sum_{\sigma \in S_{n}} \operatorname{sgn}(\sigma) \sigma_{X, Y, Z, W}\left[\sum_{\alpha \in R(I)} \alpha_{X, Y} x_{1}^{u_{1,1}} \cdots x_{n}^{u_{n, 1}} y_{1}^{u_{1,2}} \cdots y_{n}^{u_{n, 2}}\right. \\
& \left.\partial_{z_{1}}^{v_{1,1}} \cdots \partial_{z_{n}}^{v_{n, 1}} \partial_{w_{1}}^{v_{1,2}} \cdots \partial_{w_{n}}^{v_{n, 2}} \Delta_{T}(Z, W)\right]
\end{aligned}
$$

With these equations, we can begin the process of showing that the collection $\mathcal{B} \mathcal{A} \mathcal{Q}_{S, T}$ spans $\mathbb{C}_{S, T}^{-}[X, Y, Z, W]$ with coefficients from $\mathbb{C}$.

\section{Lemma 5.6.}

If $S$ and $T$ are dense then the collection

$$
\mathcal{C S C O}_{S, T}=\left\{[U, V]_{\text {per }}^{-}: U \in \mathcal{C S}_{S}, V \in \mathcal{C O}_{T}, \operatorname{sh}(U)=\operatorname{sh}(V)\right\}
$$

spans $\mathbb{C}_{S, T}^{-}[X, Y, Z, W]$ with coefficients from $\mathbb{C}$.

\section{Proof}

Given any monomial $q(Z, W) \in \mathbb{C}[Z, W]$, Lemma 5.3 and Theorem 3.8 imply that

$$
q(Z, W)=\sum_{k} c_{k}\left[I_{k}, V_{k}\right]_{p e r}\left(\partial_{Z}, \partial_{W}\right) \Delta_{T}(Z, W)
$$

(identically), where $V_{k} \in \mathcal{C O}_{T}, I_{k} \in \mathcal{S T}_{n}, \operatorname{sh}\left(V_{k}\right)=\operatorname{sh}\left(I_{k}\right)$ and $c_{k} \in \mathcal{I}_{T}(Z, W) \cup \mathbb{C}$. Thus for any monomial

$$
p(X, Y)=x_{1}^{q_{1}} x_{2}^{q_{2}} \cdots x_{n}^{q_{n}} y_{1}^{r_{1}} y_{2}^{r_{2}} \cdots y_{n}^{r_{n}},
$$

we have

$$
\begin{aligned}
& \sum_{\sigma \in S_{n}} \operatorname{sgn}(\sigma) \sigma_{X, Y, Z, W}[p(X, Y) q(Z, W)] \\
= & \sum_{\sigma \in S_{n}} \operatorname{sgn}(\sigma) \sigma_{X, Y, Z, W}\left[p(X, Y) \sum_{k} c_{k}\left[I_{k}, V_{k}\right]_{p e r}\left(\partial_{Z}, \partial_{W}\right) \Delta_{T}(Z, W)\right]
\end{aligned}
$$




$$
\begin{aligned}
& =\sum_{k} c_{k} \sum_{\sigma \in S_{n}} \operatorname{sgn}(\sigma) \sigma_{X, Y, Z, W}\left[x_{1}^{q_{1}} x_{2}^{q_{2}} \cdots x_{n}^{q_{n}} y_{1}^{r_{1}} \cdots y_{n}^{r_{n}}\left[I_{k}, V_{k}\right]_{p e r}\left(\partial_{Z}, \partial_{W}\right) \Delta_{T}(Z, W)\right] \\
& =\sum_{k} c_{k}\left[U_{k}, V_{k}\right]_{\text {per }}^{-}
\end{aligned}
$$

by equation (5.22). Note that in equation (5.24) that it is possible for some of the $U_{k}$ to not be column-strict. Using the algorithm associated to Theorem 2.1 and equations (5.22) and (5.23), we know that

$$
\sum_{k} c_{k}\left[U_{k}, V_{k}\right]_{\text {per }}^{-}=\sum_{i} d_{i}\left[P_{i}, Q_{i}\right]_{\text {per }}^{-}
$$

where all of the $P_{i}$ and $Q_{i}$ are column-strict. Note that if $r(Z, W) \in \mathcal{I}_{T}(Z, W)$ then we must have $r(Z, W)[P, Q]_{\text {per }}^{-} \in \mathcal{I}_{S, T}^{-}(X, Y, Z, W)$. Additionally, note that if either $P \notin \mathcal{C S}_{S}$ or $Q \notin \mathcal{C S}_{T}$ then $[P, Q]_{\text {per }}^{-} \in \mathcal{I}_{S, T}^{-}(X, Y, Z, W)$. Without loss of generality, we may assume that $P_{i} \in \mathcal{C S}_{S}$ and $Q_{i} \in \mathcal{C S}_{T}$. Thus the collection

$$
\mathcal{C S C S}_{S, T}=\left\{[P, Q]_{\text {per }}^{-}: P \in \mathcal{C S}_{S}, Q \in \mathcal{C S}_{T_{\psi}}, \operatorname{sh}(P)=\operatorname{sh}(Q)\right\}
$$

spans $\mathbb{C}_{S, T}^{-}[X, Y, Z, W]$ with coefficients from $\mathbb{C}$.

Let

$$
S=\left\{s_{1}, s_{2}, \cdots, s_{n}\right\}
$$

and

$$
T=\left\{t_{1}, t_{2}, \cdots, t_{n}\right\}
$$

(recall that $S$ and $T$ are the sets from which $\Delta_{S}(X, Y)$ and $\Delta_{T}(Z, W)$ are constructed listed in increasing order with respect to $\left.<_{\mathcal{A}}\right)$. Let $s^{\prime}=\min \left\{s_{1},(0,1)\right\}$ and $t^{\prime}=$ $\min \left\{t_{1},(0,1)\right\}$. Let $U$ and $V$ be the tableaux of shape $n$ with

$$
U=s^{\prime} \quad s^{\prime} \quad \cdots \quad s^{\prime} .
$$

and

$$
V=t^{\prime} \quad t^{\prime} \quad \cdots \quad t^{\prime}
$$

For any pair of tableaux $(M, N)$ such that $(M, N)>_{\text {str }}(U, V)$ (see equation (3.14) and equation (4.6)), we have that $[M, N]_{\overline{p e r}}^{-} \in \mathcal{I}_{S, T}^{-}(X, Y, Z, W)$ since

$$
\begin{aligned}
& {[M, N]_{p e r}^{-}\left(\partial_{X}, \partial_{Y}, \partial_{Z}, \partial_{W}\right) \Delta_{S}(X, Y) \Delta_{T}(Z, W) } \\
= & \frac{1}{f_{I}} \sum_{\sigma \in S_{n}} \operatorname{sgn}(\sigma) \sigma_{X, Y, Z, W}\left[\left[[I, M]_{p e r}\left(\partial_{X}, \partial_{Y}\right) \Delta_{S}(X, Y)\right]\right. \\
& {\left.\left[\left[[I, N]_{p e r}\left(\partial_{Z}, \partial_{W}\right) \Delta_{T}(Z, W)\right]\left(\partial_{Z}, \partial_{W}\right) \Delta_{T}(Z, W)\right]\right] } \\
= & 0 .
\end{aligned}
$$


Now suppose that $(P, Q)$ is the largest pair of column-strict tableaux in $\mathcal{C} \mathcal{S C S} \mathcal{S}_{S, T}$ with respect to $<_{s t r}$ (see equation (4.6)) such that $[P, Q]_{\overline{p e r}}^{-}$is not in the linear span of $\mathcal{C S C O}_{S, T}$ (with coefficients from $\mathbb{C}$ ) and $[P, Q]_{\text {per }}^{-} \notin \mathcal{I}_{S, T}^{-}(X, Y, Z, W)$. Particularly, we must have that $Q \notin \mathcal{C} \mathcal{O}_{T}$. Using Theorem 3.7 we have

$$
\begin{aligned}
& {[I, Q]_{p e r}\left(\partial_{Z}, \partial_{W}\right) } \\
= & m_{\gamma_{2}}\left(\partial_{Z}, \partial_{W}\right)\left[I, C_{2}\right]_{p e r}\left(\partial_{Z}, \partial_{W}\right)-\sum_{\substack{M>s t r \\
s h(M)=s h(Q)}} c_{M}[I, M]_{p e r}\left(\partial_{Z}, \partial_{W}\right) \\
- & \sum_{\operatorname{sh}\left(M^{\prime}\right)>_{L} \operatorname{sh}(Q)} c_{I^{\prime}, M^{\prime}}\left[I^{\prime}, M^{\prime}\right]_{p e r}\left(\partial_{Z}, \partial_{W}\right)
\end{aligned}
$$

identically, where $C_{2}=C_{\rho_{Q}}(s t(Q))$ and $\gamma_{2}=\gamma_{\rho_{Q}, Q}$. Now,

$$
\begin{aligned}
& {[P, Q]_{\text {per }}^{-}} \\
& =\sum_{\sigma \in S_{n}} \operatorname{sgn}(\sigma) \sigma_{X, Y, Z, W}\left[[I, P]_{p e r}(X, Y)[I, Q]_{p e r}\left(\partial_{Z}, \partial_{W}\right) \Delta_{T}(Z, W)\right] \\
& =\sum_{\sigma \in S_{n}} \operatorname{sgn}(\sigma) \sigma_{X, Y, Z, W}\left[[I, P]_{p e r}(X, Y) m_{\gamma_{2}}\left(\partial_{Z}, \partial_{W}\right)\left[I, C_{2}\right]_{p e r}\left(\partial_{Z}, \partial_{W}\right) \Delta_{T}(Z, W)\right] \\
& -\sum_{\sigma \in S_{n}} \operatorname{sgn}(\sigma) \sigma_{X, Y, Z, W}\left[[I, P]_{p e r}(X, Y) \sum_{\substack{M>s t r Q \\
\operatorname{sh}(M)=\operatorname{sh}(Q)}} c_{M}[I, M]_{p e r}\left(\partial_{Z}, \partial_{W}\right) \Delta_{T}(Z, W)\right] \\
& -\sum_{\sigma \in S_{n}} \operatorname{sgn}(\sigma) \sigma_{X, Y, Z, W}\left[[I, P]_{p e r}(X, Y) \sum_{s h\left(M^{\prime}\right)>_{L} \operatorname{sh}(Q)} c_{I^{\prime}, M^{\prime}}\left[I^{\prime}, M^{\prime}\right]_{p e r}\left(\partial_{Z}, \partial_{W}\right) \Delta_{T}(Z, W)\right] .
\end{aligned}
$$

Notice that

$$
\sum_{\sigma \in S_{n}} \operatorname{sgn}(\sigma) \sigma_{X, Y, Z, W}\left[[I, P]_{p e r}(X, Y) m_{\gamma_{2}}\left(\partial_{Z}, \partial_{W}\right)\left[I, C_{2}\right]_{p e r}\left(\partial_{Z}, \partial_{W}\right) \Delta_{T}(Z, W)\right]=0
$$

since $m_{\gamma_{2}}\left(\partial_{Z}, \partial_{W}\right) \Delta_{T}(Z, W)=0$ and thus

$$
\begin{aligned}
& \sum_{\sigma \in S_{n}} \operatorname{sgn}(\sigma) \sigma_{X, Y, Z, W}\left[[I, P]_{p e r}(X, Y) m_{\gamma_{2}}\left(\partial_{Z}, \partial_{W}\right)\left[I, C_{2}\right]_{p e r}\left(\partial_{Z}, \partial_{W}\right) \Delta_{T}(Z, W)\right] \\
& \in \mathcal{I}_{S, T}^{-}(X, Y, Z, W) .
\end{aligned}
$$

Both

$$
\sum_{\sigma \in S_{n}} \operatorname{sgn}(\sigma) \sigma_{X, Y, Z, W}\left[[I, P]_{p e r}(X, Y) \sum_{\substack{M>s s t r \\ s h(M)=s h(Q)}} c_{Q}[I, M]_{p e r}\left(\partial_{Z}, \partial_{W}\right) \Delta_{T}(Z, W)\right]
$$

and

$$
\sum_{\sigma \in S_{n}} \operatorname{sgn}(\sigma) \sigma_{X, Y, Z, W}\left[[I, P]_{p e r}(X, Y) \sum_{s h\left(M^{\prime}\right)>_{L} \operatorname{sh}(Q)} c_{I^{\prime}, M^{\prime}}\left[I^{\prime}, M^{\prime}\right]_{p e r}\left(\partial_{Z}, \partial_{W}\right) \Delta_{T}(Z, W)\right]
$$


lead to sums of bipermanents that are larger than $(P, Q)$ with respect to $>_{s t r}$. The former is true since it is summed over $M$ such that $M>_{s t r} Q$. The latter uses equation (5.22) and the fact that

$$
\begin{aligned}
& {[I, P]_{p e r}(X, Y)\left[I^{\prime}, M^{\prime}\right]_{p e r}\left(\partial_{Z}, \partial_{W}\right) \Delta_{T}(Z, W) } \\
= & \sum_{i} c_{i} p(x, y)\left[I^{\prime}, M^{\prime}\right]_{p e r}\left(\partial_{Z}, \partial_{W}\right) \Delta_{T}(Z, W) .
\end{aligned}
$$

This implies that $[P, Q]_{\text {per }}^{-}$is in the span of $\mathcal{C S C O}_{S, T}$ as well as the fact that $\mathcal{C S C O}_{S, T}$ spans $\mathbb{C}_{S, T}^{-}[X, Y, Z, W]$ with coefficients from $\mathbb{C}$.

Let $[U, V]_{\text {per }}^{-} \notin \mathcal{B} \mathcal{A} \mathcal{Q}_{S, T}$ be the largest bipermanent with respect to $>_{\text {str }}$ (see equation (4.6)) with $U \in \mathcal{C S}_{S}$, and $V \in \mathcal{C O}_{T}$ such that $[U, V]_{\text {per }}^{-}$is not in the linear span of $\mathcal{B} \mathcal{A} \mathcal{Q}_{S, T}$ with coefficients from $\mathbb{C}$. Now if $U \notin \mathcal{C O}_{S}$, by Theorem 3.7 we can find a

$$
\gamma_{1}=\gamma_{\rho_{U}, U}(X, Y) \in \Gamma_{S_{\psi}}
$$

such that

$$
\begin{aligned}
& {[I, U]_{\text {per }}} \\
& =m_{\gamma_{1}}(X, Y)[I, C]_{p e r}-\sum_{\substack{M>s t r U \\
s h(M)=s h(U)}} c_{M}[I, M]_{p e r}-\sum_{s h\left(M^{\prime}\right)>_{L} \operatorname{sh}(U)} c_{M^{\prime}}\left[I^{\prime}, M^{\prime}\right]_{p e r}(5
\end{aligned}
$$

where $C=C_{\rho_{U}}(s t(U))$ and $c_{M}, c_{M^{\prime}} \in \mathbb{C}$. Therefore,

$$
\begin{aligned}
& {[U, V]_{p e r}^{-}} \\
& =\frac{1}{f_{I}} \sum_{\sigma \in S_{n}} \operatorname{sgn}(\sigma) \sigma_{X, Y, Z, W}\left[[I, U]_{p e r}(X, Y)[I, V]_{p e r}\left(\partial_{Z}, \partial_{W}\right) \Delta_{T}(Z, W)\right] \\
& =\frac{1}{f_{I}} \sum_{\sigma \in S_{n}} \operatorname{sgn}(\sigma) \sigma_{X, Y, Z, W} \\
& {\left[m_{\gamma_{1}}(X, Y)[I, C]_{p e r}(X, Y)[I, V]_{p e r}\left(\partial_{Z}, \partial_{W}\right) \Delta_{T}(Z, W)\right]} \\
& -\frac{1}{f_{I}} \sum_{\sigma \in S_{n}} \operatorname{sgn}(\sigma) \sigma_{X, Y, Z, W} \\
& {\left[\sum_{\substack{M>s t r U \\
\text { sh(M)=sh(U) }}} c_{M}[I, M]_{p e r}(X, Y)[I, V]_{p e r}\left(\partial_{Z}, \partial_{W}\right) \Delta_{T}(Z, W)\right]} \\
& -\frac{1}{f_{I}} \sum_{\sigma \in S_{n}} \operatorname{sgn}(\sigma) \sigma_{X, Y, Z, W} \\
& {\left[\sum_{s h\left(M^{\prime}\right)>_{L} \operatorname{sh}(U)} c_{I^{\prime}, M^{\prime}}\left[I^{\prime}, M^{\prime}\right]_{p e r}(X, Y)[I, V]_{p e r}\left(\partial_{Z}, \partial_{W}\right) \Delta_{T}(Z, W)\right] .}
\end{aligned}
$$


Once again,

$$
\begin{aligned}
& \sum_{\sigma \in S_{n}} \operatorname{sgn}(\sigma) \sigma_{X, Y, Z, W}\left[m_{\gamma_{1}}(X, Y)[I, C]_{p e r}(X, Y)[I, V]_{p e r}\left(\partial_{Z}, \partial_{W}\right) \Delta_{T}(Z, W)\right] \\
& \in \mathcal{I}_{S, T}^{-}(X, Y, Z, W) .
\end{aligned}
$$

The terms

$$
\sum_{\sigma \in S_{n}} \operatorname{sgn}(\sigma) \sigma_{X, Y, Z, W}\left[\sum_{\substack{M>s s t U \\ \operatorname{sh}(M)=s h(U)}} c_{M}[I, M]_{p e r}(X, Y)[I, V]_{p e r}\left(\partial_{Z}, \partial_{W}\right) \Delta_{T}(Z, W)\right]
$$

and

$$
\begin{aligned}
& \sum_{\sigma \in S_{n}} \operatorname{sgn}(\sigma) \sigma_{X, Y, Z, W} \\
& {\left[\sum_{\operatorname{sh}\left(M^{\prime}\right)>_{L} \operatorname{sh}(U)} c_{I^{\prime}, M^{\prime}}\left[I^{\prime}, M^{\prime}\right]_{p e r}(X, Y)[I, V]_{p e r}\left(\partial_{Z}, \partial_{W}\right) \Delta_{T}(Z, W)\right]}
\end{aligned}
$$

correspond to sums of bipermanents that are larger than $[U, V]_{\text {per }}^{-}$with respect to $>_{\text {str }}$ by equation (5.23). Therefore, $[U, V]_{p e r}^{-}$can be written as a linear combinations of elements of

$$
\mathcal{B A} \mathcal{Q}_{S, T}=\left\{[U, V]_{\text {per }}^{-}: U \in \mathcal{C} \mathcal{O}_{S}, V \in \mathcal{C O}_{T} \text { and } \operatorname{sh}(U)=\operatorname{sh}(V)\right\}
$$

with coefficients in $\mathbb{C}$. Thus we have the following theorem.

\section{Theorem 5.7.}

If $S$ and $T$ are dense then the collection $\mathcal{B} \mathcal{A} \mathcal{Q}_{S, T}$ spans $\mathbb{C}_{S, T}^{-}[X, Y, Z, W]$ with coefficients from $\mathbb{C}$. Hence, $\mathcal{B} \mathcal{A} \mathcal{Q}_{S, T}$ is a basis for $\mathbb{C}_{S, T}^{-}[X, Y, Z, W]$ with coefficients from $\mathbb{C}$.

Now, the bidegree of $\left[I, C_{\rho}(U)\right]_{p e r}\left(\partial_{Z}, \partial_{W}\right) \Delta_{T}(Z, W)$ (with $I$ standard) (as a polynomial in $\mathbb{C}[Z, W]$ ) equals the bidegree of $\left[I^{t}, C_{\rho^{-}}\left(U^{t}\right)\right]_{\text {per }}$ (recall Theorem 3.4). Recall that $d_{\psi_{S}}(q, t)$ is a polynomial defined in equation (3.21) and that $\Upsilon_{\psi_{S}}$ and $\Upsilon_{\psi_{T}}$ are collections of sequences defined in equation (3.4). Thus, Theorem 5.7 immediately yields the following.

\section{Corollary 5.8.}

If $S$ and $T$ are dense then the Hilbert series $\mathcal{H}\left(\mathbb{C}_{S, T}^{-}[X, Y, Z, W]\right)$ of $\mathbb{C}_{S, T}^{-}[X, Y, Z, W]$ is given by

$$
\begin{aligned}
& \mathcal{H}\left(\mathbb{C}_{S, T}^{-}[X, Y, Z, W]\right) \\
& =\sum_{\lambda \vdash n} \sum_{\rho \in \Upsilon_{\psi_{S}}} \sum_{\rho^{\prime} \in \Upsilon_{\psi_{T}}} \sum_{(M, N) \in \mathcal{S} \mathcal{T}_{\lambda} \times \mathcal{S} \mathcal{T}_{\lambda}} t^{\left|C_{\rho, 1}(M)\right|} q^{\left|C_{\rho, 2}(M)\right|} r^{\left|C_{\rho^{\prime}, 1}\left(N^{t}\right)\right|} s^{\left|C_{\rho^{\prime}, 2}\left(N^{t}\right)\right|} \\
& =d_{\psi_{S}}(q, t) d_{\psi_{T}}(s, r) \sum_{\lambda \vdash n} \sum_{\substack{(M, N) \in \mathcal{S} \mathcal{T}_{\lambda} \times \mathcal{S} \mathcal{T}_{\lambda} \\
\rho=\left(0^{n}\right), \rho^{\prime}=\left(0^{n}\right)}} t^{\left|C_{\rho, 1}(M)\right|} q^{\left|C_{\rho, 2}(M)\right|} r^{\left|C_{\rho^{\prime}, 1}\left(N^{t}\right)\right|} s^{\left|C_{\rho^{\prime}, 2}\left(N^{t}\right)\right|},
\end{aligned}
$$


where $\mathcal{S} \mathcal{T}_{\lambda}$ denotes the collection of standard tableaux of shape $\lambda, C_{\rho}(M)$ denotes a cocharge tableau corresponding to a tableau $M$ and a sequence $\rho,\left|C_{\rho, 1}(M)\right|$ and $\left|C_{\rho, 2}(M)\right|$ denote the sums of the first and second coordinates of the cocharge tableau $C_{\rho}(M)$, respectively, and $N^{t}$ denotes the transpose of the tableau $N$.

\section{Example}

With $S=T$ given in (3.19) we have

$$
\begin{aligned}
& \mathcal{H}\left(\mathbb{C}_{S, T}^{-}[X, Y, Z, W]\right) \\
= & (1+t)\left(r s+r^{2} s\right)+\left(t+q+t q+t^{2}\right)\left(r+s+r s+r^{2}\right)+\left(t q+t^{2} q\right)(1+r) \\
= & (1+t)(1+r)[r s+(t+q)(r+s)+t q] .
\end{aligned}
$$

Note that the dimensions (as vector spaces) of $\mathbb{C}_{S, T}^{+}[X, Y, Z, W]$ and $\mathbb{C}_{S, T}^{-}[X, Y, Z, W]$ are equal. Both of these dimensions are equal to $d_{\psi_{S}} d_{\psi_{T}} n$ ! (see equation (3.20)).

\section{References}

[1] E. E. Allen, A conjecture of Procesi and a new basis for the decomposition of the graded left regular representation of $S_{n}$, Adv. in Math. 100 (1993), 262-292.

[2] E. E. Allen, The descent monomials and a basis for the diagonally symmetric polynomials, Journal of Algebraic Combinatorics 3 (1994), 5-16.

[3] E. E. Allen, Bitableaux bases for the diagonally invariant polynomial quotient rings, Adv. in Math. 130 (1997), 242-260.

[4] E. E. Allen, Some graded representations of the complex reflection groups, Journal of Combinatorial Theory, Series A 87 (1999), 287-332.

[5] E. E. Allen and M. E. Cox, Garsia-Haiman modules: themes and variations, in preparation.

[6] F. Bergeron, A. Garsia and G. Tesler, Multiple left regular representations generated by alternants, Journal of Combinatorial Theory, Series A 91 (2000), 49-83.

[7] J. Désarménian, J. P. Kung and G. C. Rota, Invariant theory, Young tableaux and combinatorics, Advances in Mathematics 27, (1978), 63-92.

[8] A. M. Garsia, Combinatorial methods in the theory of Cohen-Macaulay rings, Adv. in Mathematics 38 (1980), 229-266.

[9] A. Garsia, Personal communication.

[10] A. Garsia and M. Haiman, A graded representation model for Macdonald's polynomials, Proc. Natl. Acad. Sci. USA 90 (April 1993), 3607-3610. 
[11] F. Grosshans, G. C. Rota and J. Stein, "Invariant Theory and Superalgebras", Conference Board of Mathematics, Number 69, 1987.

[12] M. Haiman, Hilbert schemes, polygraphs, and the Macdonald positivity conjecture. Journal of the A.M.S., 14 2001, 941-1006 (electronic).

[13] Hungerford, "Algebra", Graduate Texts in Mathematics 73 Springer-Verlag, New York-Berlin, 1974.

[14] A. Lascoux and M. P. Schützenberger, Polynomes de Schubert, C.R. Acad. Sci Paris 294 (1982), 447-450.

[15] I. G. Macdonald, "Schubert Polynomials", classroom notes, UCSD, La Jolla, CA (1990).

[16] V. Reiner, "Quotients of Coxeter Complexes and P-Partitions", Memoirs of the AMS, Number 460, January 1992.

[17] Rotman, "Introduction to the Theory of Groups", fourth edition, Graduate Texts in Mathematics 148, , Springer-Verlag, New York, 1995.

[18] R. Steinberg, Differential equations invariant under finite reflection groups, Trans. Amer. Math. Soc. 112 (1964), 392-400. 